\title{
A single trapped Rydberg ion
}

\section{Gerard Higgins}

Academic dissertation for the Degree of Doctor of Philosophy in Physics at Stockholm University to be publicly defended on Friday 27 April 2018 at 10.00 in sal FA32, AlbaNova universitetscentrum, Roslagstullsbacken 21.

\begin{abstract}
Systems of trapped ions and systems of ultracold Rydberg atoms are used at the forefront of quantum physics research and they make strong contenders as platforms for quantum technologies. Trapped Rydberg ions are a new hybrid technology envisaged to have both the exquisite control of trapped ion systems and the strong interactions of Rydberg atoms.

In this work a single trapped Rydberg ion is experimentally investigated. A trapped ${ }^{88} \mathrm{Sr}^{+}$ion is excited to Rydberg states using two ultraviolet lasers. Effects of the strong trapping electric fields on the sensitive Rydberg ion are studied. After mitigating unwanted trap effects, the ion is coherently excited to Rydberg states and a quantum gate is demonstrated. This thesis lays much of the experimental groundwork for research using this novel system.
\end{abstract}

Keywords: Trapped ions, Rydberg physics, quantum information platforms, atomic physics.

Stockholm 2018

http://urn.kb.se/resolve?urn=urn:nbn:se:su:diva-153415

ISBN 978-91-7797-179-5

ISBN 978-91-7797-180-1

\section{Department of Physics}

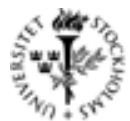

Stockholm University, 10691 Stockholm 

GERARD HIGGINS

A SINGLE TRAPPED RYDBERG ION 



\section{universität innsbruck}

\section{A SINGLE TRAPPED RYDBERG ION}

\section{GERARD HIGGINS}

A dissertation submitted in partial fulfilment of the requirements for the double degree (cotutelle)

Doctor of Philosophy (Physics) at the University of Innsbruck, Austria and

Doctor of Philosophy (Physics) at Stockholm University, Sweden.

Carried out under the supervision of Assoc. Prof. Markus Hennrich at the Department of Experimental Physics, Innsbruck and the Department of Physics, Stockholm.

March 2018 
COLOPHON

(C) Gerard Higgins, Stockholm University 2018.

ISBN (print) 978-91-7797-179-5

ISBN (online) 978-91-7797-180-1

Printed in Sweden by Universitetsservice US-AB, Stockholm 2018.

Distributor: Department of Physics, Stockholm University.

This document was typeset using the typographical look-and-feel classicthesis developed by André Miede and Ivo Pletikosić. The style was inspired by Robert Bringhurst's "The Elements of Typographic Style". classicthesis is available for both LTEX and LYX:

https://bitbucket.org/amiede/classicthesis/

Hermann Zapf's Palatino and Euler typefaces (Type 1 PostScript fonts URW Palladio L and FPL) are used for the body text, and Palatino Sans is used for figures. 


\section{ABSTRACT}

Systems of trapped ions and systems of ultracold Rydberg atoms are used at the forefront of quantum physics research and they make strong contenders as platforms for quantum technologies. Trapped Rydberg ions are a new hybrid technology envisaged to have both the exquisite control of trapped ion systems and the strong interactions of Rydberg atoms.

In this work a single trapped Rydberg ion is experimentally investigated. A trapped ${ }^{88} \mathrm{Sr}^{+}$ion is excited to Rydberg states using two ultraviolet lasers. Effects of the strong trapping electric fields on the sensitive Rydberg ion are studied. After mitigating unwanted trap effects, the ion is coherently excited to Rydberg states and a quantum gate is demonstrated. This thesis lays much of the experimental groundwork for research using this novel system.

\section{SAMMANFATTNING}

System med fångade joner och system med ultrakalla Rydbergatomer används i framkanten av kvantfysikforskning, och är starka kandidater som plattformar för kvantteknologier. Fångade Rydbergjoner är en ny hybridteknologi som förutses kunna kontrollera system med fångade joner, och samtidigt ha Rydbergatomers starka interaktioner.

I detta arbete undersöks en ensam fångad Rydbergjon experimentellt. En fångad ${ }^{8} \mathrm{Sr}^{+}$-jon exciteras till Rydbergtillstånd med två ultravioletta lasrar. Effekter från de starka elektriska fångstfälten på de känsliga Rydbergjonerna undersöks. Efter dämpning av fällans oönskade effekter exciteras jonen koherent till Rydbergtillstånd och en kvantgrind demonstreras. Denna avhandling utgör en experimentell grund för forskning som använder detta nya system. 
Systeme gefangener Ionen und ultrakalter Rydbergatome finden Anwendung im Spitzenfeld quantenphysikalischer Forschung. Beide sind vielversprechende Kandidaten für quantentechnologische Anwendungen. Gefangene Rydbergionen bilden eine neuartige, hybride Technologie. Sie versprechen, die außerordentlichen Kontrollmöglichkeiten gefangener Ionen mit den starken Wechselwirkungen von Rydbergatomen zu kombinieren.

Die vorliegende Arbeit befasst sich mit der experimentellen Untersuchung eines einzelnen gefangenen Rydbergions. Ein gefangenes ${ }^{88} \mathrm{Sr}^{+}$Ion wird mittels zweier ultravioletter Laser in Rydbergzustände angeregt, wobei die Auswirkungen der starken elektrischen Fallenfelder auf das empfindliche Rydbergion untersucht werden. Nachdem unerwünschte Falleneffekte hinreichend abgeschwächt sind, wird das Ion kohärent in einen Rydbergzustand angeregt sowie ein Quantengatter demonstriert. Diese Doktorarbeit legt einen Großteil des technischen Grundstocks für zukünftige Forschung mit diesem neuartigen System. 
Two journal publications have resulted from the experiments presented in this thesis. My contributions to the publications are described below.

Higgins, G., Li, W., Pokorny, F., Zhang, C., Kress, F., Maier, C., Haag, J., Bodart, Q., Lesanovsky, I. \& Hennrich, M. Single Strontium Rydberg Ion Confined in a Paul Trap. Phys. Rev. X 7, 021038 (Jun 2017).

I set up the experiment together with colleagues, I measured the experimental data with assistance from colleagues, and I analysed the data and wrote the manuscript together with colleagues.

Higgins, G., Pokorny, F., Zhang, C., Bodart, Q. \& Hennrich, M. Coherent Control of a Single Trapped Rydberg Ion. Phys. Rev. Lett. 119, 220501 (Nov 2017).

I made improvements to the setup together with colleagues, I measured the experimental data with assistance from Chi, I extended theory calculations, I analysed the data together with Chi and Markus, and I wrote the manuscript with assistance from Markus. 

First and foremost I thank my supervisor, Markus Hennrich. I count myself lucky having a supervisor who is always aware of what is going on in the lab and who provides constant, patient guidance.

From the time when the lab was empty, it has been a pleasure to work with Fabian Pokorny. Thank you for your steadfast good cheer and unwavering calm. I also thank Christine, Hannes, Florian, Quentin, Chi and Andreas for many good times together in the lab.

During my time in Innsbruck I was fortunate to be part of the Blatt group, in which expertise is generously shared. In particular I wish to thank Matthias Brandl for advice about electronics, Muir Kumph for guidance in building stable optical resonators, Esteban Martinez for answering countless questions about trapped ion experiments, Michael Niedermayr for gold-coating the trap electrodes, Ben Ames for help crystallising ions, the senior scientists Christian Roos, Tracy Northup and Yves Colombe for sharing their experience; and Rainer Blatt for welcoming me into his group. I also thank the members of the electronic and mechanical workshops for much assistance.

I wish to thank the administrative staff, both in Innsbruck and in Stockholm, for help with bureaucratic issues; particular thanks are due to Patricia Moser and Isa Callderyd Öhman. I would also like to thank Sandra Scherl, Per-Erik Tegnér and Georg Moser for help arranging the cotutelle agreement.

I thank the theorists Weibin Li and Igor Lesanovsky at the University of Nottingham for a fruitful collaboration.

I thank Nadine, Gabriel, Esteban, Martin, Slava, Philip, Bojana and Bárbara in Innsbruck and Massi, Irina, Vani, Colin, Belu, Tania and Iara in Stockholm for friendship.

Thank you Cris for support and for so many adventures; I love you to bits.

Lastly I thank my mother for always encouraging me and for making me feel confident. 

I INTRODUCTION I

1.1 Trapped atomic ions 1

1.2 Rydberg atoms 4

1.3 Trapped Rydberg ions 6

1.3.1 Theoretical investigations 7

1.3.2 Experimental realisations 9

2 PROPERTIES OF STRONTIUM RYDBERG IONS 13

2.1 Electronic structure 13

2.2 Atomic wavefunctions 14

2.3 Length scales 15

2.4 State lifetime 16

2.5 Rydberg excitation Rabi frequency $\quad 17$

2.6 Two-qubit Rydberg gate 18

3 EXPERIMENTAL SETUP 21

3.1 Experiments with a single trapped ion 21

3.1.1 Laser-driven transitions 21

3.1.2 Laser cooling 27

3.1.3 State initialisation 29

3.1.4 Measurement 31

3.2 Linear Paul trap 32

3.2.1 Ions in the trapping potential 33

3.2.2 Trap components 34

3.2.3 Trap temperature 34

3.2.4 Photo electrons induced by UV light 35

3.2.5 Motional heating 35

3.2.6 Magnetic field 36

3.2.7 Ion loading 36

3.2.8 Micromotion compensation $\quad 37$

3.3 Laser systems 38

3.3.1 $\quad$ Laser sources $\quad 38$ 
3.3.2 Laser pulse generation using AOMs $\quad 4^{1}$

3.3.3 Optical fibres 41

3.3.4 Laser frequency stabilisation $\quad 41$

3.4 Electronics for experimental control 44

4 TWO-PHOTON RYDBERG EXCITATION 47

4.1 The first excitation step 47

4.1.1 $4 \mathrm{D}_{5 / 2} \leftrightarrow 6 \mathrm{P}_{3 / 2}$ transition 47

4.1.2 $4 \mathrm{D}_{3 / 2} \leftrightarrow 6 \mathrm{P}_{1 / 2}$ transition 49

4.2 Focussing Rydberg excitation lasers on an ion $\quad 5^{1}$

4.2.1 UV laser setups at the experiment chamber $\quad 51$

4.2.2 Focussing $243 \mathrm{~nm}$ light on an ion 51

4.2.3 Focussing $\sim 307 \mathrm{~nm}$ light on an ion 52

4.3 Two-photon resonance condition 53

4.4 Counterpropagating Rydberg-excitation lasers 54

4.5 Zeeman-split Rydberg S-state resonances 56

4.6 Rydberg energy series $\quad 58$

5 ION LOSS BY DOUBLE IONISATION 61

5.1 Obstacles presented by ion loss 61

5.2 Measurements of the loss product 62

5.2.1 Imaging ions with an EMCCD camera 62

5.2.2 Measuring the axial mode frequency by tickling electrodes $\quad 64$

6 TRAP EFFECTS 67

6.1 Effects on highly-polarisable Rydberg ions 67

6.1.1 With overlapping quadrupole field nulls 68

6.1.2 With non-overlapping quadrupole field nulls $\quad 72$

6.2 Effects on Rydberg ions with large quadrupole moments 79

6.2.1 Theory behind the trap-induced coupling 80

6.2.2 Floquet theory 83

6.2.3 Experimental results 85

7 COHERENT EXCitATION OF RYDBERG STATES 89

7.1 The three-level system 89 
7.2 Autler-Townes effect 91

7.3 Two-photon Rabi oscillations 93

7.3.1 Theoretical background 94

7.3.2 Experimental results 95

7.4 Stimulated Raman adiabatic passage $\quad 98$

7.4.1 Coherent Rydberg excitation using STIRAP 99

7.4.2 Measurement of a Rydberg state lifetime 103

7.4.3 Geometric phase measurements 104

8 SUMMARY AND OUTLOOK III

BIBLIOGRAPHY

113 
Figure 1.1

Figure 2.1

Figure 2.2

Figure 2.3

Figure 2.4

Figure 2.5

Figure 3.1

Figure 3.2

Figure $3 \cdot 3$

Figure 3.4

Figure 3.5

Figure 3.6

Figure 3.7

Figure 3.8

Figure 3.9

Figure 4.1

Figure 4.2

Figure 4.3

Figure 4.4

Figure 4.5

Figure 4.6

Figure 4.7

Figure 5.1

Figure 5.2

Figure $5 \cdot 3$

Figure 6.1

Figure 6.2
${ }^{88} \mathrm{Sr}^{+}$and ${ }^{40} \mathrm{Ca}^{+}$level schemes 10

Rydberg energy levels $\quad 14$

Length scales of a trapped Rydberg ion system 15

Rydberg state lifetimes $\quad 16$

Rydberg excitation Rabi frequency $\quad 18$

Rydberg ions interaction strength and Rydberg gates 20

${ }^{88} \mathrm{Sr}^{+}$level scheme $\quad 22$

Transitions between electronic and motional states $\quad 24$ Ion trap 26

Sideband cooling $\quad 28$

Phonon number state preparation 31

Electron shelving $\quad 33$

String of ${ }^{88} \mathrm{Sr}^{+}$ions $\quad 34$

Typical laser setup $\quad 38$

Electronic control system $\quad 44$

First Rydberg excitation step: $4 \mathrm{D}_{5 / 2} \leftrightarrow 6 \mathrm{P}_{3 / 2} \quad 48$

First Rydberg excitation step: $4 \mathrm{D}_{3 / 2} \leftrightarrow 6 \mathrm{P}_{1 / 2} \quad 50$

$243 \mathrm{~nm}$ laser focus on the ion 52

$307 \mathrm{~nm}$ laser focus on the ion 53

Two-photon excitation of Rydberg S-state

Zeeman effect with Rydberg S-states $\quad 57$

${ }^{88} \mathrm{Sr}^{+}$Rydberg S-state energy series 59

Mixed-species crystals imaged on a camera 63

Mixed-species crystals motional mode frequen-

cies 65

Resonant driving of motional modes 66

Phonon-number dependence of Rydberg res-

onance frequency $\quad 7^{1}$

Effect of phonon distribution on Rydberg res-

onance linewidth $\quad 72$ 
Figure 6.3 Overlap of phonon states of a ground state ion and a Rydberg ion $\quad 76$

Figure 6.4 Trap-induced coupling of electronic and motional degrees of freedom 77

Figure 6.5 Micromotion compensation using Rydberg spectroscopy 78

Figure 6.6 Effects of electric quadrupole fields on Rydberg states with large electric quadrupole moments

Figure 6.7 Quadrupole-field-induced coupling in the Floquet basis $\quad 84$

Figure 6.8 Observation of quadrupole-field-induced coupling between Rydberg states 86

Figure 7.1 Relevant level scheme for coherent Rydberg excitation 90

Figure 7.2 Autler-Townes effect 92

Figure 7.3 Avoided crossing of dressed states eigenenergies 93

Figure 7.4 Two-photon Rabi oscillations 96

Figure 7.5 STIRAP pulse sequences 101

Figure 7.6 Rydberg excitation by STIRAP and $42 S_{1 / 2}$ lifetime $\quad 102$

Figure 7.7 Accumulation of geometric phase during Rydberg excitation process 106

Figure 7.8 Process tomography of a Rydberg ion phase gate 109

Table 1.1

Rydberg state properties 4

Table 4.1 ${ }^{88} \mathrm{Sr}^{+} \mathrm{S}$-state energy series 60 


\section{ACRONYMS}

AOM acousto-optic modulator

DDS direct digital synthesizer

EMCCD electron-multiplying charge-coupled device

MW microwave

PDH Pound-Drever-Hall

PMT photo-multiplier tube

RF radiofrequency

SFG sum frequency generation

SHG second harmonic generation

STIRAP stimulated Raman adiabatic passage

UV ultraviolet

VUV vacuum-ultraviolet 


\section{INTRODUCTION}

In the last decades researchers have achieved exquisite control of different quantum systems. This has allowed fundamental tests to be carried out, including studies of quantum measurements [1, 2], quantum contextuality [3] and the quantum wavefunction [4, 5]; as well as Bell test experiments [6-8]. New technologies which take advantage of highly-controlled quantum systems are being pursued [9], and proof of principle devices capable of quantum computation [10, 11], quantum simulation [12], quantum communication [13] and quantum metrology [14] have been demonstrated.

Some systems are more suitable than others as platforms for particular quantum technologies, just as some systems are more suitable than others for carrying out particular fundamental tests. For instance, the long coherence times of trapped ion qubits make for excellent quantum memories [15], while the propagation speed of photonic qubits allows the locality loophole to be closed in Bell test experiments. My thesis is concerned with a new experimental platform, namely a system of trapped Rydberg ions. This platform combines two established systems: trapped atomic ions and Rydberg atoms. In this opening chapter aspects of the two constituent technologies are summarised before trapped Rydberg ions are introduced.

\section{I TRAPPED ATOMIC IONS}

Atomic ions may be trapped in electromagnetic fields. The trap configuration typically used for quantum information purposes is called a linear Paul trap [16]. In such a trap a string of ions is confined by a combination of oscillating and static electric fields. Quantum bits (qubits) are stored in electronic states of the ions. Qubits are coherently manipulated using lasers (and sometimes microwaves) which drive transitions between electronic states. Ions can be well isolated 
from the environment; they are trapped in ultra-high vacuum, usually around $30 \mu \mathrm{m}$ to $300 \mu \mathrm{m}$ from any surface [17]. Owing in part to this, trapped ion qubits show excellent coherence properties; coherence times of several minutes have been demonstrated [15]. Ions can be confined for days in the deep potential of a Paul trap [18].

Ion qubits can be prepared, manipulated and read-out with high fidelity [19]. Entanglement operations between ion qubits can be carried out with low errors [20,21]. A system of 14 trapped ion qubits holds the record for the largest genuine multipartite entangled state stored on separate particles. ${ }^{1}$ The trapped ion architecture is a leading contender for quantum computation and simulations.

Quantum simulations with trapped ions have allowed researchers to study exotic phenomena, such as particle-antiparticle production in a lattice gauge field model [23], a discrete time crystal [24], manybody localisation [25] and a dynamical phase transition involving 53 spins [26]. Systems of trapped ions have been used to simulate open quantum systems [27], to study statistical mechanics in quantum systems [28-30] and to investigate thermodynamics at the level of a single atom [31]. They may be employed in the future for experimental investigations in the emerging field of quantum thermodynamics. Quantum simulators are also of commercial interest; they may be utilised to find molecular energies and this may assist pharmaceutical research [32]. A trapped ion quantum simulator has already been used to find the energies of a simple diatomic molecule [33].

Trapped ions have been used as proof-of-principle quantum computers. Of the various algorithms proposed for quantum computers, Shor's factorisation algorithm has generated the most interest, since it could be used to break public-key cryptography schemes. While condensed versions of this algorithm have been demonstrated on various platforms [34-37], the first scalable demonstration was carried out with trapped ions [11]. Various quantum error correction codes have been demonstrated in systems of trapped ions [38-40], this shows

1 A recent preprint reports a genuine multipartite entangled state of 18 qubits stored on 6 photons [22]. 
fault-tolerant quantum computing may be feasible if technological hurdles are overcome.

Trapped ions may be employed in the future for quantum communication. The workings of a quantum network in which photons transport quantum information between trapped ion memories has been demonstrated [13].

Another potential application of trapped ions is in quantum metrology; entangled states have been engineered for precision spectroscopy with enhanced sensitivity [41] and with less susceptibility to noise [42]. Further, some of the most accurate atomic clocks are trapped ion systems $[43,44]$.

Trapped ion quantum computers and simulators have yet to outperform classical computers. One of the sticking points is that manipulation of the entanglement of trapped ion qubits becomes more difficult as the number of ions in a string is increased. Entanglement manipulation in trapped ion systems commonly involves addressing of individual motional modes [45]. As the number of ions in a string is increased the number of motional modes increases and longer laser pulses are required for individual modes to be frequency-resolved [46]. Entanglement manipulation may also be carried out by coupling electronic degrees of freedom to many motional modes simultaneously, however this strategy also requires longer laser pulses as the number of ions in a string is increased [47]. As the duration of quantum operations increases, errors from decoherence become significant and quantum operations become unfeasible.

To scale up trapped ion quantum systems to the point where they out-perform classical computers, several groups are working towards a 'quantum charge-coupled device' architecture in which ions are shuttled between interconnected traps and the entanglement of small numbers of ion qubits are manipulated at a time [48]. Such an architecture likely involves a two-dimensional trapping geometry and may be better suited for simulation of a two-dimensional quantum system than the one-dimensional trapping geometry of a single linear Paul trap [12].

An alternate path to a scalable system was proposed by Müller et al. [49]. They suggest combining trapped ion and Rydberg atom systems to give a novel platform, the experimental investigation of which is 
the topic of this thesis. Neutral Rydberg atoms are introduced in the next section and the proposal from Müller et al. is described in Section 1.3.1.

\subsection{RYDBERG ATOMS}

Atomic states with high principal quantum numbers $n \gg 1$ are called Rydberg states. Rydberg atoms have exaggerated properties which follow scaling relations. Some of these properties are presented in Table 1.1.

\begin{tabular}{lcc}
\hline Property & $n$-scaling & Z-scaling \\
\hline Binding energy $E_{n}$ & $n^{-2}$ & $Z^{2}$ \\
Energy separation $E_{n+1}-E_{n}$ & $n^{-3}$ & $Z^{2}$ \\
Fine structure splitting & $n^{-3}$ & $Z^{4}$ \\
Orbital size $\langle r\rangle$ & $n^{2}$ & $Z^{-1}$ \\
Electric quadrupole moment $\Theta \sim\left\langle\mathrm{r}^{2}\right\rangle$ & $n^{4}$ & $Z^{-2}$ \\
Natural lifetime $\tau_{\text {nat }}$ & $n^{3}$ & $Z^{-4}$ \\
Blackbody radiation limited lifetime $\tau_{\mathrm{BBR}}$ & $\mathrm{n}^{2}$ & $\mathrm{Z}^{-4}$ \\
Transition dipole moment of Rydberg ex- & $\mathrm{n}^{-3 / 2}$ & $\mathrm{Z}^{-1}$ \\
citation transition $\langle g|\operatorname{ler}| \mathrm{nLJ}\rangle$ & & \\
Transition dipole moment $\left\langle\mathrm{nL} \mathrm{L}^{\prime} \mathrm{J}^{\prime}|\mathrm{er}| \mathrm{nLJ}\right\rangle$ & $\mathrm{n}^{2}$ & $\mathrm{Z}^{-1}$ \\
Dipole-dipole interaction strength & $\mathrm{n}^{4}$ & $\mathrm{Z}^{-2}$ \\
Electric polarisability $\rho$ & $\mathrm{n}^{7}$ & $\mathrm{Z}^{-4}$ \\
Van der Waals coefficient $\mathrm{C}_{6}$ & $\mathrm{n}^{11}$ & $\mathrm{Z}^{-6}$ \\
\hline
\end{tabular}

Table 1.1: Scaling of properties of Rydberg atoms and ions in terms of the principal quantum number $n$ and the core charge (also called effective nuclear charge) $Z$ [50, 51]. $Z=+1$ for neutral Rydberg atoms, $Z=+2$ for Rydberg ions with $+\mathrm{e}$ overall charge.

Much of the current research with Rydberg atoms employs strong, long-range, dipole-dipole interactions between them [52]. Depending on the separation between Rydberg atoms and the relation between the Rydberg states employed, dipole-dipole interactions are manifest 
as van der Waals interactions, Förster interactions or resonant dipoledipole interactions. Individual trapped atoms excited to stronglyinteracting Rydberg states have been used for the first deterministic generation of entanglement between two neutral atoms [53], for twoqubit Rydberg gates [54] and for experimental investigations of manybody spin models [55]. Atoms have been individually trapped and excited to Rydberg states from arrays of optical tweezers, arrays of magnetic traps and from optical lattices [56]. Two-dimensional trapping configurations are often used, which, in terms of quantum computing and simulation, has scalability advantages over the one-dimensional configuration typical of trapped ion systems.

In a Rydberg atom quantum computer qubits are encoded in Zeeman sublevels or hyperfine sublevels of the ground states of atoms. Single-qubit rotations are carried out using microwaves (MWs) or by two-photon Raman transitions, and two-qubit manipulations are carried out using the strong interactions between atoms excited to Rydberg states.

Neutral Rydberg atom quantum computers and simulators have technological hurdles that need to be overcome before they can compete with trapped ion quantum computers and simulators, let alone out-perform classical computers. A major obstacle in these systems is loss of ground state atoms from the trapping potential and atom loss during Rydberg excitation; these challenges are reviewed in [56]. Atom loss during Rydberg excitation also poses a problem in trapped Rydberg ion systems, as is discussed in Chapter 5 .

Optical nonlinearities are generally too weak for nonlinear optical phenomena, such as photon-photon gates, to be observed at the fewphoton level. Strong interactions between Rydberg atoms mean that strong optical nonlinearities can be experienced by just a few photons resonant with a transition to a Rydberg state in an atomic cloud [57]. ${ }^{2}$

2 While Rydberg-mediated nonlinear quantum optics is an exciting research field, it is unlikely to be closely linked to the novel trapped Rydberg ion system explored in this thesis. This is because trapped ion systems have much lower optical depths than atomic clouds. However, cavities could conceivably be used to enhance coupling between ions and either ultraviolet (UV) Rydberg-excitation photons or MW photons which couple Rydberg states [58, 59], to allow for nonlinear quantum optics mediated by Rydberg ions. 
Strong Rydberg interactions give rise to exotic states of matter; as a result of strong interaction between Rydberg atoms and ground state atoms weakly-bound molecules have been produced with bond lengths of thousands of Bohr radii [6o], and with giant electric dipole moments [61].

In terms of metrology, Rydberg atoms have potential as quantumenhanced sensors of electric fields [14] and as surface probes [62, 63] owing to their high electric polarisabilities. High transition dipole moments between Rydberg states make Rydberg atoms excellent sensors of weak radiofrequency $(\mathrm{RF})$ fields [64].

\subsection{TRAPPED RYDBERG IONS}

Compared with Rydberg atoms, Rydberg ions have historically received little attention. The valence electron of a positively-charged ion, with core charge (also called effective nuclear charge) $Z=+2$, is more tightly bound than the valence electron in an atom, which has $Z=+1$. Thus, more energy is required for excitation of Rydberg ions than for Rydberg atoms. Other differences between Rydberg atom and Rydberg ion properties in terms of $Z$ are shown in Table 1.1.

Rydberg ion spectra were first studied systematically in the eighties [65-67]. Atoms from atomic beams were photoionised using pulsed lasers and then excited to Rydberg states via multi-photon transitions. Rydberg ions were then doubly-ionised and detected. These studies were motivated by a desire to provide insights into "planetary atoms" with two Rydberg electrons, which are difficult to model because of electron correlations [67].

The high sensitivity of Rydberg states to electric fields and the prohibitive Rydberg excitation energy requirements did little to encourage experimentalists to excite trapped ions to Rydberg states. This changed after a theoretical investigation by Müller et al. [49] predicted trapping of Rydberg ions is feasible and strong interactions between Rydberg-excited ions may be used for fast quantum gates. This prompted two experiments to strive for trapped Rydberg ions; it has also encouraged further theoretical investigations. The theoret- 
ical investigations are reviewed in Section 1.3.1 and the experiments are introduced and compared in Section 1.3.2.

\subsubsection{Theoretical investigations}

In 2008 Müller et al. [49] predicted it is feasible to Rydberg-excite ions confined in a linear Paul trap, despite the high sensitivity of Rydberg states to electric fields (see Table 1.1). Although strong field gradients $\left(\sim 10^{9} \mathrm{~V} \mathrm{~m}^{-2}\right)$ are used for trapping, ions are well localised to within tens of nanometres from the electric field null. Müller et al. predicted for Rydberg ions up to at least $n=50$ the electric field of the ionic core dwarfs the field of the trap and Rydberg electrons are bound to the ionic core.

They did, however, predict two effects of the trap on the Rydberg ion, which are studied in Chapter 6. Firstly, Rydberg ions experience a different trapping potential to low-lying ions due to their large electric polarisabilities, and so the trapping frequencies in Rydberg states are altered. This causes unwanted entanglement to be generated between electronic and motional degrees of freedom of ions during Rydberg excitation. Secondly, states with $\mathrm{J}>\frac{1}{2}$ have giant electric quadrupole moments and the strong electric quadrupole fields used for ion trapping strongly couple such Rydberg states. Müller et al. suggest mitigating this second trap effect by using states with $\mathrm{J}=\frac{1}{2}$ : $n \mathrm{~S}_{1 / 2}$ and $\mathrm{nP} \mathrm{P}_{1 / 2}$.

Müller et al. pointed out that although van der Waals interactions are significantly weaker between Rydberg ions than they are between neutral Rydberg atoms (see Table 1.1), sizeable interactions between Rydberg ions may be generated by using MW radiation to couple Rydberg $n S_{1 / 2}$ and $n^{\prime} P_{1 / 2}$ states. The MW-dressed states can have large dipole moments which rotate with the frequency of the MW radiation. Two MW-dressed Rydberg ions may then interact strongly via dipoledipole interaction. Müller et al. proposed a trapped ion quantum computer in which qubits are stored in low-lying electronic states and two-qubit gates are carried out by exciting ion qubits to stronglyinteracting Rydberg states, similar to the Rydberg gates implemented 
in a system of neutral atoms [54]. ${ }^{3}$ Such Rydberg gates are envisaged to be fast (see Section 2.6) and offer an alternate route towards a scalable trapped ion quantum computer or simulator. They also proposed a scheme for simulating a spin model in a chain of trapped Rydberg ions.

To mitigate unwanted entanglement between electronic and motional degrees of freedom of ions which is generated during Rydberg excitation (discussed by Müller et al. [49]), in 2014 Li et al. [68] proposed engineering interacting MW-dressed Rydberg states with vanishing electric polarisabilities. Such dressed states should exist because $n S_{1 / 2}$ and $n^{\prime} P_{1 / 2}$ states have polarisabilities with opposite signs. 4 Unfortunately such dressed states have non-maximal dipole moments and thus they do not produce the strongest interactions.

In $2013 \mathrm{Li}$ et al. [69] proposed shaping the motional mode spectrum of an ion crystal using Rydberg ions. They showed that the motional mode structure of a linear string of 100 ions is significantly altered when two ions are excited to Rydberg states, due to the altered trapping potential experienced by Rydberg ions [49]. Motional modes appear which are localised on the ions that lie between the Rydberg ions. The entanglement between these Rydberg-flanked ions may then be manipulated using the common protocol in which the localised motional modes are used as an intermediary [45]. After deexcitation from the Rydberg state the vibrational mode spectrum returns to normal. In this proposal Rydberg ions are not used directly for qubit manipulation, but rather for segmenting the string. In 2015 Nath et al. [70] suggested using the shaping of the motional mode spectrum by Rydberg ions as a way to implement a quantum magnetism simulation in a two-dimensional ion crystal [70].

In a linear Paul trap ions may form linear strings or zigzag crystals, depending on the ratio of the radial mode frequencies and the axial mode frequency. A transition between these two structures can be

3 Rydberg states are not used for storing qubits because the lifetimes of the low-lying states typically used as qubits $(\sim 1 \mathrm{~s})$ are around five orders of magnitude longer than Rydberg states with $n=50(\sim 10 \mu \mathrm{s})$, while similar Rabi frequencies are used to manipulate low-lying states and to excite Rydberg states $(\sim 2 \pi \times 1 \mathrm{MHz})$.

4 Throughout this thesis Russell-Saunders term symbols $\mathrm{L}_{\mathrm{J}}$ describe total angular momentum quantum numbers. The multiplicity $2 S+1=2$ is omitted. 
induced by changing the radial mode frequencies. In $2012 \mathrm{Li}$ et al. [71] proposed starting with a system close to the structural transition and inducing the structural phase transition by exciting an ion to a Rydberg state, then reversing the transition by deexcitation. The transition is induced because the Rydberg ion experiences altered trapping frequencies [49]. Large coherent forces accompany the transition and a range of potential applications are detailed in the proposal.

The principle behind Rydberg mode shaping and Rydberg-induced structural phase transitions was experimentally demonstrated in [72], though Rydberg ions were not used. Instead ${ }^{\circ} \mathrm{Ca}^{+}$ions were irreversibly doubly-ionised and a ${ }^{\circ} \mathrm{Ca}^{+}$ion crystal was compared with a mixed species ion crystal of ${ }^{\circ} \mathrm{Ca}^{+}$and ${ }^{40} \mathrm{Ca}^{2+}$.

\subsubsection{Experimental realisations}

Trapped ions are excited to Rydberg states in two experiments: ${ }^{\circ} \mathrm{Ca}^{+}$ ions are used in the group of Ferdinand Schmidt-Kaler at the University of Mainz and ${ }^{88} \mathrm{Sr}^{+}$ions are excited in the group of Markus Hennrich, which started in 2012 at the University of Innsbruck and moved to Stockholm University in 2015. This thesis was carried out in the ${ }^{88} \mathrm{Sr}^{+}$experiment.

Both ion species are alkaline earth metals, with one valence electron. Both have $S_{1 / 2}$ ground states and metastable $D_{3 / 2}$ and $D_{5 / 2}$ states with lifetimes $\sim 1 \mathrm{~s}$. Neither species has a hyperfine structure, and in each species optical qubits are stored in a Zeeman sublevel of the $S_{1 / 2}$ ground state and a Zeeman sublevel of the metastable $D_{5 / 2}$ state. In both experiments ions are excited to Rydberg states from either of the D-states.

In the ${ }^{40} \mathrm{Ca}^{+}$experiment ions are excited to Rydberg states by driving a single-photon transition using vacuum-ultraviolet (VUV) laser light between $122 \mathrm{~nm}$ and $123 \mathrm{~nm}$. From the metastable 3D-states electric dipole transition selection rules allow excitation to either Rydberg P- or Rydberg F-states. Thus far they have excited only Rydberg F-states; they do not know why P-states have been unattainable.

In the ${ }^{88} \mathrm{Sr}^{+}$experiment we excite ions in a two-photon excitation scheme using $243 \mathrm{~nm}$ and $304 \mathrm{~nm}$ to $309 \mathrm{~nm}$ ultraviolet (UV) laser light. 
Excitation proceeds from the metastable 4D-states to Rydberg S- or Rydberg D-states using excitation laser light which is detuned from resonance with the intermediate 6P-states, as shown in Fig. 1.1.
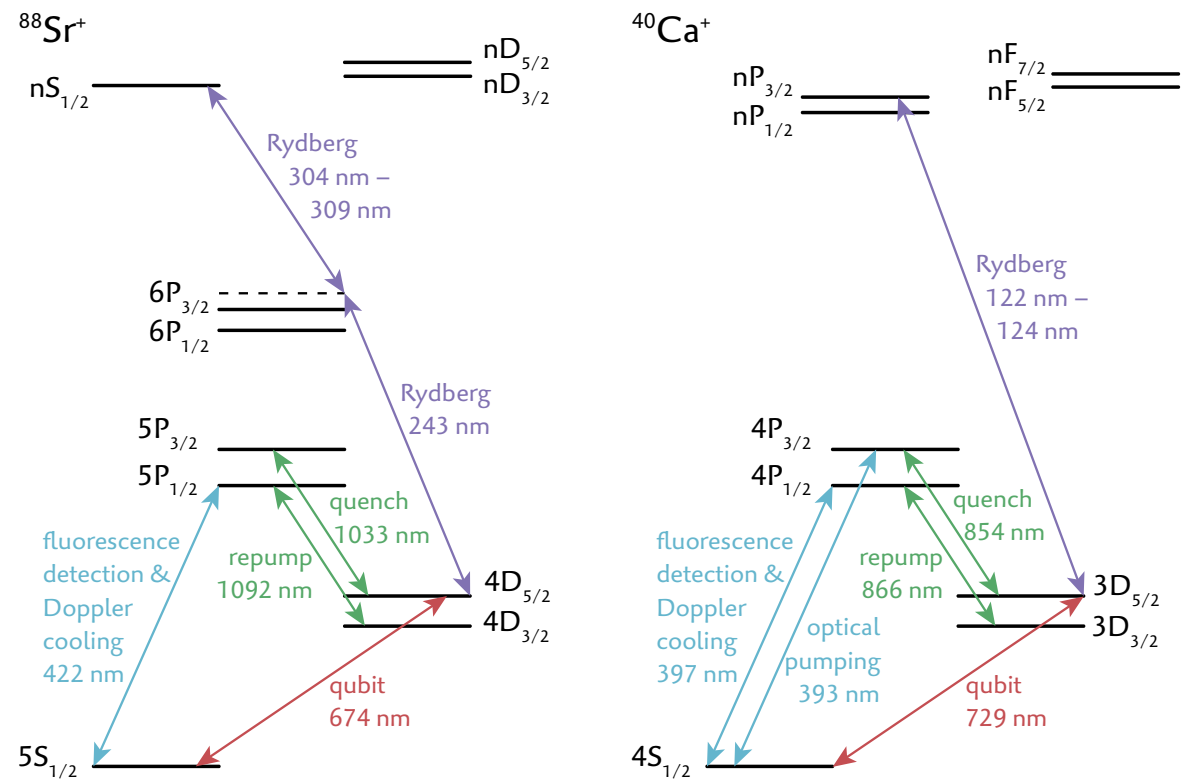

Figure 1.1: The ion species and laser systems in the two trapped Rydberg ion experiments. In Stockholm S- and D-Rydberg states of ${ }^{8} \mathrm{Sr}^{+}$ are excited via two-photon transitions, while in Mainz P- and FRydberg states of ${ }^{40} \mathrm{Ca}^{+}$may be excited via single-photon transitions. In both systems qubits are stored in sublevels of the ground state and the metastable $\mathrm{D}_{5 / 2}$ state.

The ${ }^{4} \mathrm{Ca}^{+}$experiment began first and achieved the first trapped Rydberg ions [73, 74]. Further progress in this experiment has been impeded by the broad linewidths of their Rydberg resonance lines $(\approx 2 \pi \times 4 \mathrm{MHz}$ for $22 \mathrm{~F}, 2 \pi \times 60 \mathrm{MHz}$ for $52 \mathrm{~F}$ [75]), which makes investigation of trap effects difficult and coherent excitation of Rydberg states unobtainable. The linewidths have technical contributions, including the VUV laser linewidth, uncompensated electric dipole fields which perturb the Rydberg state, and the poorly-defined VUV laser polarisation which causes multiple non-degenerate transitions to be driven - these transitions are not individually resolved. The linewidths are also Doppler broadened because the relatively high mo- 
mentum of a VUV photon makes it difficult to access the Lamb-Dicke regime (Section 3.1.1). Additionally the oscillating electric trapping field couples different Rydberg states, this leads to non-resolved Floquet sidebands (Section 6.2) which also contribute to the linewidth of the $52 \mathrm{~F}$ resonance. A further experimental obstacle is the difficulty of maintaining the VUV Rydberg-excitation laser.

In the ${ }^{88} \mathrm{Sr}^{+}$experiment we have progressed further. We measure narrower Rydberg resonances $(\approx 2 \pi \times 300 \mathrm{kHz}$, likely limited by the Rydberg-excitation lasers linewidths) thanks to our state-of-the-art ion trap (Section 3.2), the counter-propagating two-photon Rydberg excitation setup (Section 4.4), addressing of individual transitions (Section 4.5) and near-ground-state cooling which allows effects of the trapping electric fields on the highly-polarisable Rydberg ion to be mitigated (Section 6.1). The narrow Rydberg resonances allow us to investigate effects of the trap on a Rydberg ion (Chapter 6) and to coherently excite Rydberg states (Chapter 7).

Our experiment proceeds despite an objection raised to the twophoton excitation scheme; higher laser powers are required in a multiphoton excitation scheme, which in turn make ion loss by photoionisation more likely [76]. Ion loss is the topic of Chapter 5 . 



\section{PROPERTIES OF STRONTIUM RYDBERG IONS}

In this chapter the electronic structure of Rydberg ions is described before key properties are reviewed. The relevant length scales in a Rydberg ion system are described in Section 2.3, Rydberg state lifetimes are presented in Section 2.4, and the interaction strength of MW-dressed Rydberg ions and the fidelity of a two-qubit Rydberg gate are discussed in Section 2.6.

\section{I ELECTRONIC STRUCTURE}

${ }^{88} \mathrm{Sr}^{+}$is a multi-electron system; 37 electrons orbit the nucleus of charge +38 . Rydberg states are well-described by a single valence electron orbiting a spherical core with charge $Z=+2$, since the other 36 electrons form closed shells and shield the nuclear charge. Rydberg states of ${ }^{88} \mathrm{Sr}^{+}$thus follow the Rydberg scaling relations in Table 1.1. Because the valence electron wavefunction extends inside the core, the core electrons do not completely shield the nucleus. As a result the scaling relations are followed in terms of the effective principal quantum number $n^{*}=n-\mu$, where the quantum defect $\mu$ is determined empirically. ${ }^{1} \mu$ depends on the atomic species as well as the angular momentum quantum numbers $L$ and J. For example the binding energy formula is adapted as follows:

$$
E=-\frac{Z^{2} R}{n^{2}} \rightarrow-\frac{Z^{2} R}{(n-\mu)^{2}},
$$

where $\mathrm{R}$ is the Rydberg constant. $\mathrm{Sr}^{+}$energy series are displayed in Fig. 2.1, literature values are used for $\mu$ [67]. The ${ }^{88} \mathrm{Sr}^{+} \mathrm{nS}_{1 / 2}$ energy series is measured in this work, the results are in Section 4.6.

1 The symbol $\delta$ is commonly used for the quantum defect. In this work $\mu$ is used to avoid confusion with the partial derivative symbol in Section 4.6. 


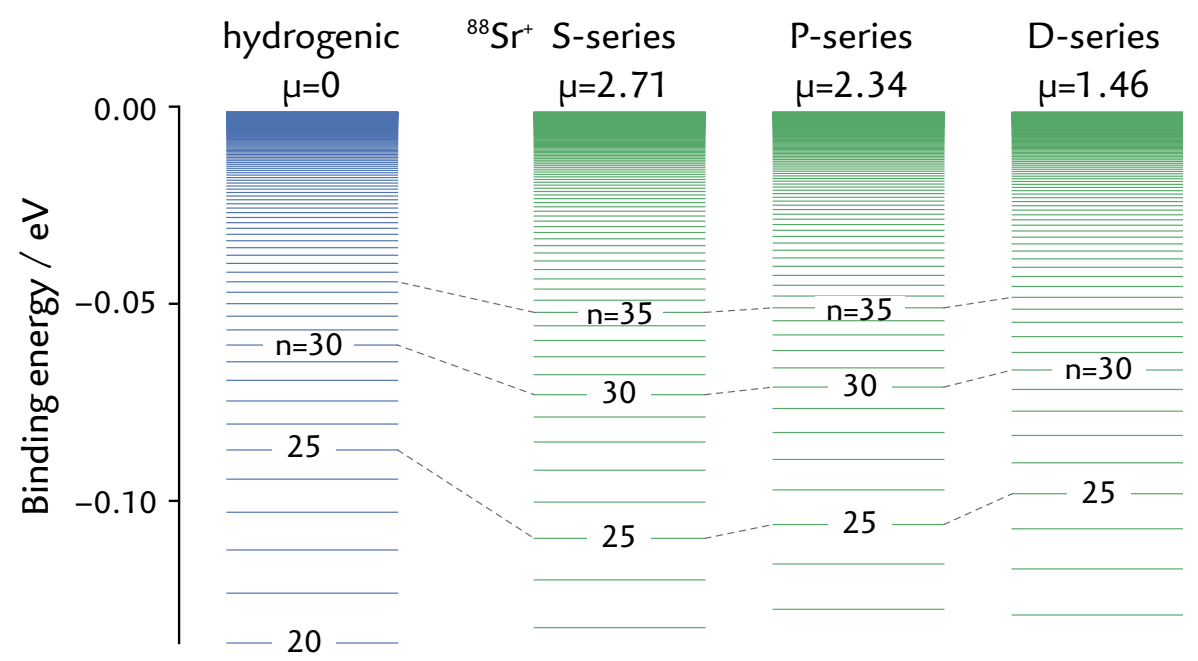

Figure 2.1: Rydberg state energies depend on the principal quantum number and the quantum defect $\mu$. The energy levels of a hydrogenlike system with $Z=+2$ and of different ${ }^{88} \mathrm{Sr}^{+}$series do not match because the quantum defects $(\mu)$ are different. Principal quantum numbers $(n)$ are labelled and $\mu$ values are taken from [67].

\subsection{ATOMIC WAVEFUNCTIONS}

Atomic wavefunctions describing the multi-electron ${ }^{88} \mathrm{Sr}^{+}$ion are derived by reducing the many-body problem to a two-body problem; the inner electrons form closed shells which screen the nuclear Coulomb potential and thus the valence electron experiences a screened Coulomb potential. The screened Coulomb potential is described by parameters from [77]. The Schrödinger equation for the valence electron moving around the core is solved numerically to give atomic wavefunctions. This method is described more completely in [76].

Wavefunctions of ${ }^{88} \mathrm{Sr}^{+}$were calculated by Weibin Li at the University of Nottingham and used to produce theoretical values of Rydberg state polarisabilities (Section 6.1), quadrupole moments (Section 6.2), and natural lifetimes (Section 7.4.2), as well as branching ratios of decays from excited states. I calculated similar wavefunctions and Rydberg state properties by adapting the Python package ARC [78] 
which was developed for calculations of neutral Rydberg atom properties.

\subsection{LENGTH SCALES}

The Rydberg orbital radius scales with $\mathrm{n}^{* 2}$, and so Rydberg ions are much bigger than ground state ions. The Rydberg ion experiments (Section 1.3.2) and most of the theory proposals (Section 1.3.1) involve a string of ions confined in a linear Paul trap (Section 3.2), with ions separated by $\sim 5 \mu \mathrm{m}$. For the Rydberg states considered in this work ( $n<60)$, the orbital radius is much less than the inter-ion spacing and thus the overlap of two Rydberg-ion wavefunctions is negligible, as shown in Fig. 2.2.

Each ion is trapped in a $3 \mathrm{D}$ pseudo-harmonic potential. Unlike point-like ground-state ions, the Rydberg orbital radius $(\approx 100 \mathrm{~nm}$ for $\left.53 S_{1 / 2}\right)$ exceeds the extent of the ion motion $(\sim 10 \mathrm{~nm})$ and trap effects specific to Rydberg ions emerge (Chapter 6).
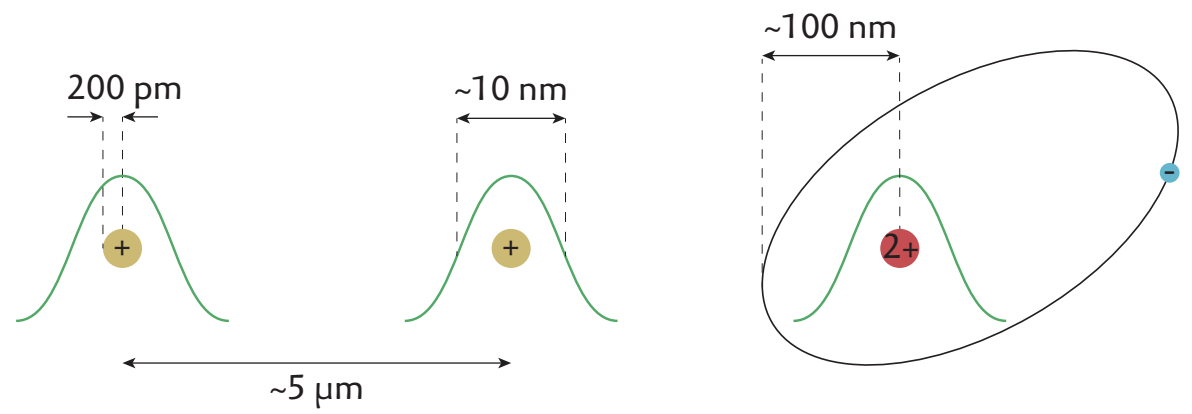

Figure 2.2: Ground state ions (yellow) are much smaller than the extent of a trapped ion's motion (green). The orbital radius of Rydberg states considered in this thesis is larger than the extent of the ion motion and smaller than the separation between two trapped ions. The Rydberg electron orbital is classically represented. The orbital radius is $\approx 100 \mathrm{~nm}$ for $53 S_{1 / 2}$. 


\subsection{STATE LIFETIME}

The natural lifetime of a trapped Rydberg ion may be shortened by transitions driven by blackbody radiation and transitions driven by the trapping electric fields.

The natural lifetime of an atomic state $m$ is given by the inverse of the sum of radiative decay rates

$$
\tau_{\mathrm{m}, \text { nat }}=\left(\sum_{\mathrm{m}^{\prime}} A_{\mathrm{m} \mathrm{m}^{\prime}}\right)^{-1},
$$

where the Einstein A-coefficient $A_{m m^{\prime}}$ is the radiative decay rate from $m$ to $m^{\prime}$. Radiative decay of Rydberg states is strongest to low-lying states. Higher Rydberg states overlap less with low-lying states and as a result higher Rydberg states have higher natural lifetimes. The natural lifetime scales as $n^{* 3}$, as shown in Fig. 2.3.

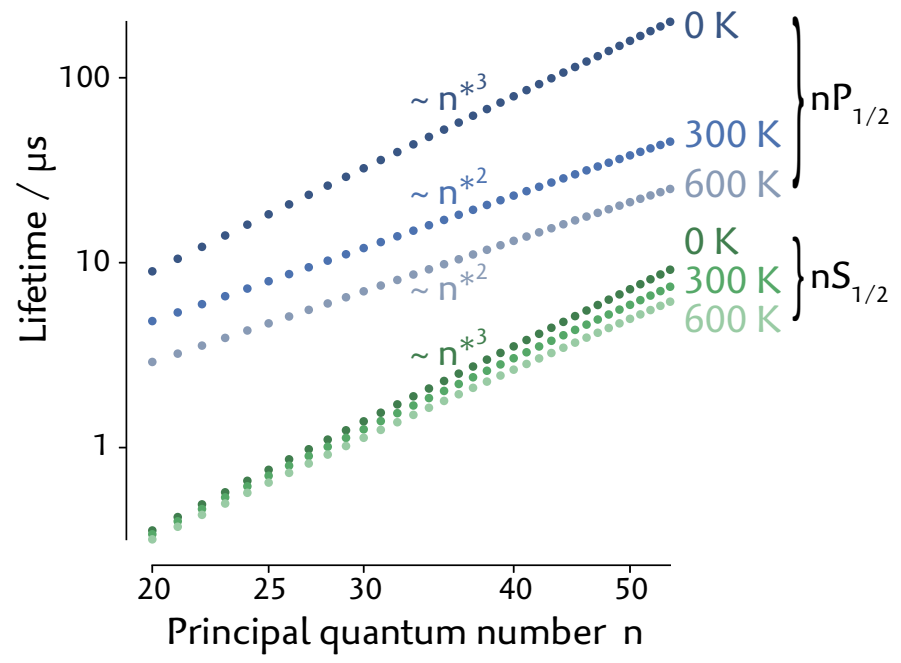

Figure 2.3: Lifetimes of Rydberg $S_{1 / 2^{-}}$and $\mathrm{P}_{1 / 2}$-states of ${ }^{88} \mathrm{Sr}^{+}$with different temperatures of surroundings. Natural lifetimes scale with $n^{* 3}$, lifetimes limited by blackbody radiation scale with $n^{* 2}$. Trap effects do not affect the lifetimes of the states which are shown. Logarithmic scales are used. 
Blackbody radiation drives transitions between states and shortens the lifetime of atomic state $\mathrm{m}$ [79]

$$
\tau_{m, B B R}=\left(\sum_{m^{\prime}} A_{m m^{\prime}}+\sum_{m^{\prime}} A_{m m^{\prime}} \bar{n}+\sum_{m^{\prime \prime}} A_{m^{\prime \prime} m} \bar{n}\right)^{-1},
$$

where the photon occupation number is

$$
\bar{n}=\left(e^{\Delta / k T}-1\right)^{-1}
$$

and $\Delta$ is the energy difference between the states $m$ and $m^{\prime}$ or $m$ and $\mathrm{m}^{\prime \prime}$. While population only moves to states lower in energy $\left(m^{\prime}\right)$ by radiative decay, blackbody radiation also drives transitions to higher-energy states $\left(\mathrm{m}^{\prime \prime}\right)$. Because of the $\bar{n}$-dependence, blackbody radiation drives transitions between energetically-similar states most strongly. Rydberg state lifetimes scale as $n^{* 2}$ if they are limited by blackbody radiation, as shown in Fig. 2.3.

The trapping electric fields strongly drive transitions between Rydberg states with $\mathrm{J}>\frac{1}{2}$, since these states have large electric quadrupole moments (see Section 6.2). The lifetimes of Rydberg states with $J>\frac{1}{2}$ are thus significantly shortened by trapping electric fields [76]. Transitions driven by typical trapping fields are negligible for $\mathrm{J}=\frac{1}{2}$ Rydberg states up to at least $n=50$ [49]; as a result $J=\frac{1}{2}$ Rydberg states have longer lifetimes, which are limited by natural decay or blackbody radiation driven transitions. Because Rydberg gate fidelities are higher for longer lived Rydberg states [80] the Rydberg ion gate proposals (Section 1.3.1) employ $n S_{1 / 2}$ and $n P_{1 / 2}$ states (which have $\mathrm{J}=\frac{1}{2}$ ).

As shown in Fig. 2.3 Rydberg states $50 \mathrm{~S}_{1 / 2}$ and $50 \mathrm{P}_{1 / 2}$ have lifetimes $\sim 10 \mu$ s when the temperature of the surroundings is $300 \mathrm{~K}$ (similar to the laboratory temperature). Coherent Rydberg excitation and Rydberg gates thus require Rabi frequencies $>\sim 2 \pi \times 1 \mathrm{MHz}$.

In Section 7.4.2 the measurement of the $42 S_{1 / 2}$ lifetime is shown.

\subsection{RYDBERG EXCITATION RABI FREQUENCY}

As the principal quantum number is increased the overlap of a Rydberg state with a low-lying state is reduced, and as a result the trans- 
ition dipole moment between a low-lying state and a Rydberg state becomes smaller. The transition dipole moment and the Rydbergexcitation Rabi frequency scale as $n^{*-3 / 2}$, as shown in Fig. 2.4.

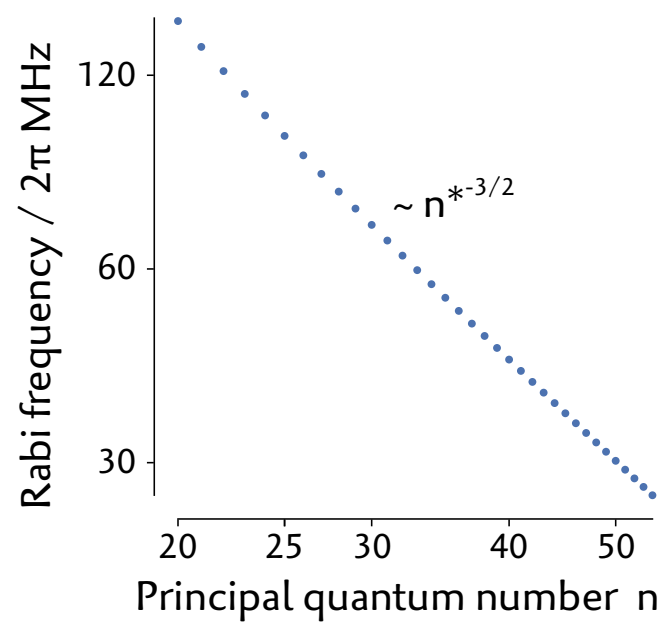

Figure 2.4: It becomes progressively more difficult to drive a transition between a low-lying state and a Rydberg state as the principal quantum number $\mathrm{n}$ is increased. The Rabi frequencies for $6 \mathrm{P}_{3 / 2}, \mathrm{~m}_{\mathrm{J}}=-\frac{3}{2} \leftrightarrow n \mathrm{nS}_{1 / 2}, \mathrm{~m}_{\mathrm{J}}=-\frac{1}{2}$ transitions driven by $10 \mathrm{~mW}$ of laser light focussed to a $10 \mu \mathrm{m}$ beam waist are shown. These transitions are constituents of the two-photon transitions used in the experiment. The plotted Rabi frequencies use calculated dipole moments of the $6 \mathrm{P}_{3 / 2}, \mathrm{~m}_{\mathrm{J}}=-\frac{3}{2} \leftrightarrow n S_{1 / 2}, \mathrm{~m}_{\mathrm{J}}=$ $-\frac{1}{2}$ transition. Logarithmic scales are used.

Provided the Rydberg state decay rate is limited by natural decay or by blackbody radiation-induced transitions, coherent excitation of higher Rydberg states may be achieved with lower laser light intensities, due to difference in scaling between the Rydberg excitation Rabi frequency $\left(n^{*-3 / 2}\right)$ and the Rydberg state decay rate $\left(n^{*-3}\right.$ for natural decay or $\mathrm{n}^{*-2}$ for blackbody radiation-induced decay).

\subsection{TWO-QUBIT RYDBERG GATE}

The Rydberg ion gate proposals (Section 1.3.1) involve coupling $n \mathrm{~S}_{1 / 2}$ and $n \mathrm{P}_{1 / 2}$ states using MW radiation. The coupled states have dipole 
moments, with magnitudes which scale as $n^{* 2}$. Orbital sizes and the transition dipole moment $\left\langle n L J|e r| n L^{\prime} J^{\prime}\right\rangle$ scale in the same fashion. The strength of the dipole-dipole interaction between two MWdressed Rydberg states thus scales as $n^{* 4}$, as shown in Fig. 2.5(a). The interaction strength also depends on the separation between the ions $\Delta z$; the strength varies as $\Delta z^{-3}$.

Two-qubit Rydberg gates using the dipole-dipole interaction are more efficient when higher Rydberg states with stronger interactions and longer lifetimes are used, ${ }^{2}$ as shown in Fig. 2.5(b). A Rydberg blockade gate is described in [8o]; the gate error is proportional to $\left(U \tau_{r}\right)^{-2 / 3}$, where $U$ is the interaction strength and $\tau_{r}$ is the Rydberg state lifetime. This gate requires higher Rydberg excitation Rabi frequencies for higher Rydberg states; the optimal Rabi frequency is proportional to $\sqrt[3]{\frac{\mathrm{u}^{2}}{\tau}}$. This means higher laser light intensities are required when higher Rydberg states are used for the blockade gate. A Rydberg interaction gate is described in [81]; the gate error is proportional to $\left(U \tau_{r}\right)^{-1}$. The future implementation of such a gate in our system is discussed at the end of Section 7.4.3.

These gates may be carried out over sub-microsecond timescales, as shown in Fig. 2.5(c). The time required for the blockade gate is inversely proportional to the Rydberg excitation Rabi frequency, while the time required for the interaction gate is inversely proportional to the interaction strength.

2 Higher principal quantum number states have longer lifetimes provided they are limited by natural decay or blackbody radiation-induced transitions. 
a

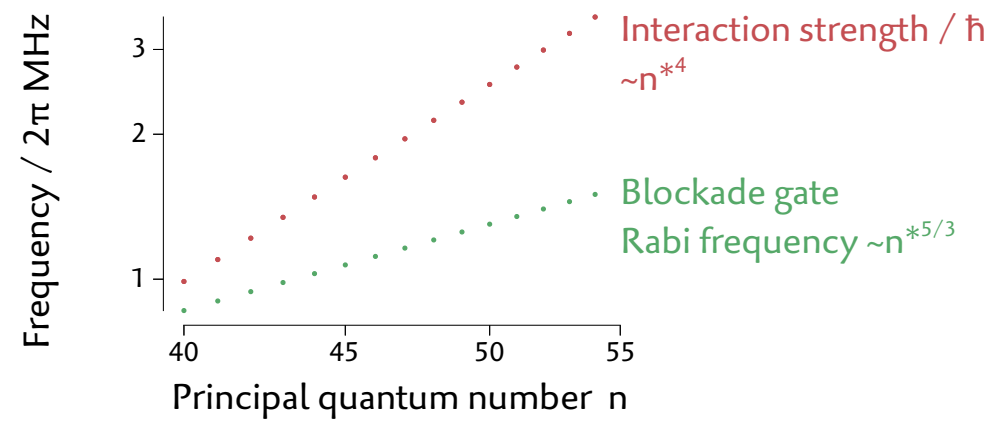

b

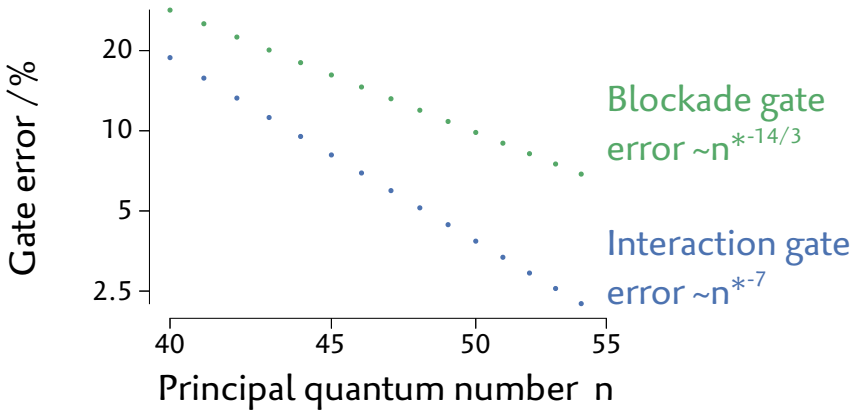

C

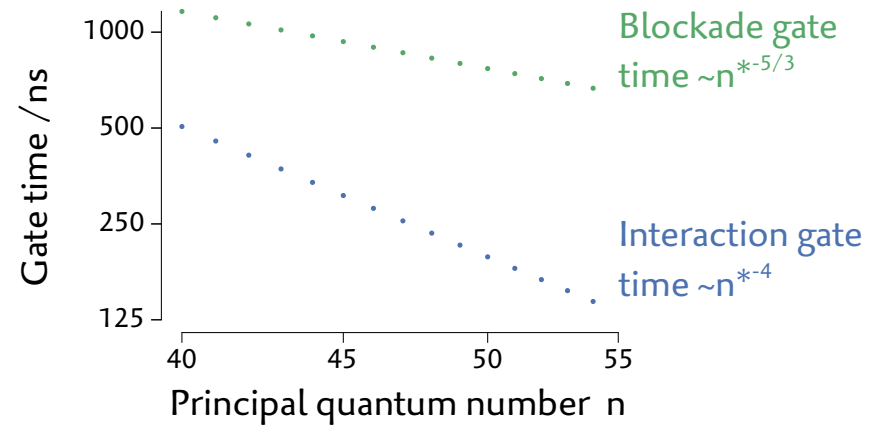

Figure 2.5: Higher Rydberg states interact more strongly and can be used for faster gates with lower errors. (a) The dipole-dipole interaction strength is calculated for two MW-dressed ions separated by $4 \mu \mathrm{m}$; the rest of the calculations use the same ion separation. For the blockade gate, higher Rydberg states require higher Rydberg excitation Rabi frequencies. (b) The gate errors are lower for higher Rydberg states with stronger interactions and longer lifetimes; the states considered here have lifetimes limited by natural decay. (c) Sub-microsecond gate times may be obtained. Logarithmic scales are used. 
This chapter deals with the main parts of the laboratory and how they come together for experiments to be carried out. During experiments trapped ions are manipulated using laser pulses. In Section 3.1 general experimental sequences are described and experimental requirements are established. In the subsequent sections systems developed to meet these requirements are presented, namely the linear Paul trap (Section 3.2), laser systems (Section 3.3) and electronic control systems (Section 3.4).

\subsection{EXPERIMENTS WITH A SINGLE TRAPPED ION}

A single trapped ion is probed in most of the experiments in this thesis. Experiments involve sequences of laser pulses which drive transitions between states of an ion. The laser-driven transitions are described in Section 3.1.1, thereafter the key steps in an experiment are introduced. A typical experiment consists of the following steps:

1. Laser cooling

2. State initialisation

3. Transitions are driven by the Rydberg-excitation lasers or the qubit laser

4. Measurement

The laser cooling, state initialisation and measurement steps are described in Sections 3.1.2, 3.1.3 and 3.1.4 respectively.

\subsubsection{Laser-driven transitions}

Within a linear Paul trap ions are confined in effective harmonic trapping potentials in the three spatial dimensions. The trapping po- 
tentials are described in more detail in Section 6.1.1. Trapped ions have electronic degrees of freedom and motional degrees of freedom; laser light is used to drive transitions between electronic and motional states.

\section{Electronic states of ${ }^{88} \mathrm{Sr}^{+}$}

The eigenenergies of different electronic states are represented in the level scheme in Fig. 3.1. A magnetic field splits the levels into Zeeman

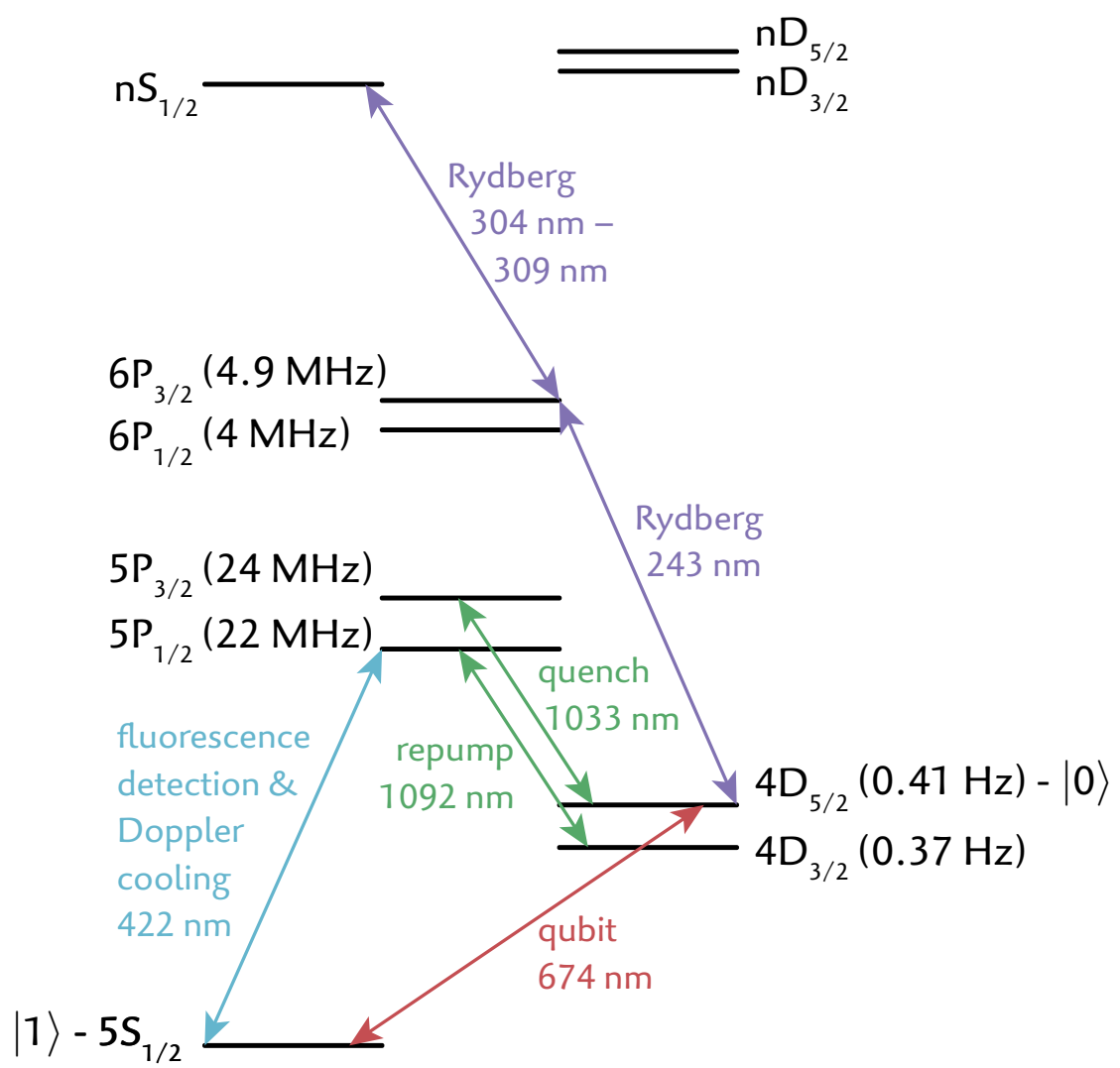

Figure 3.1: ${ }^{88} \mathrm{Sr}^{+}$level scheme including natural decay rates and the lasers used for driving transitions. Natural decay rates $\Gamma / 2 \pi$ are taken from $[82-85]$ and the $6 \mathrm{P}_{3 / 2}$ decay rate is measured in Section 4.1.1. Zeeman sublevels are not shown. 
sublevels, which are labelled by the magnetic quantum number $m_{J}$ (see Section 3.2.6).

A Zeeman sublevel of the ground state $5 S_{1 / 2}$ and a Zeeman sublevel of $4 \mathrm{D}_{5 / 2}$ are used to store an ion qubit. Electric quadrupole transitions between $5 S_{1 / 2}$ and $4 D_{5 / 2}$ sublevels are driven by $674 \mathrm{~nm}$ laser light with Rabi frequencies $\sim 2 \pi \times 100 \mathrm{kHz}$. These Rabi frequencies greatly exceed the $4 \mathrm{D}_{5 / 2}$ natural decay rate $2 \pi \times 410 \mathrm{mHz}$ [84], and thus qubit coherence times can greatly exceed quantum gate times. $5 \mathrm{~S}_{1 / 2} \leftrightarrow 4 \mathrm{D}_{5 / 2}$ transitions are also used for resolved sideband cooling (Section 3.1.2), optical pumping (Section 3.1.3), micromotion compensation (Section 3.2.8) and ion temperature measurements (described later in this section).

The rest of the lasers are used to drive electric dipole transitions. The $2 \pi \times 22 \mathrm{MHz}$ natural decay rate of $5 \mathrm{P}_{1 / 2}$ [85] means photons are scattered at a high rate when the $5 \mathrm{~S}_{1 / 2} \leftrightarrow 5 \mathrm{P}_{1 / 2}$ transition is strongly driven by $422 \mathrm{~nm}$ laser light. Owing in part to this, the $5 \mathrm{~S}_{1 / 2} \leftrightarrow 5 \mathrm{P}_{1 / 2}$ transition is used for ion measurement (which relies on detection of scattered photons, see Section 3.1.4) as well as Doppler cooling (Section 3.1.2).

When the $422 \mathrm{~nm}$ laser is employed $1092 \mathrm{~nm}$ laser light is often used to prevent optical pumping to $4 \mathrm{D}_{3 / 2}$, which is caused by decay from $5 \mathrm{P}_{1 / 2}$ to $4 \mathrm{D}_{3 / 2}$ in $6 \%$ of cases [86]. $1033 \mathrm{~nm}$ laser light is used to remove population from $4 \mathrm{D}_{5 / 2}$ and initialise the system (Section 3.1.3), it is also used for carrying out resolved sideband cooling.

Laser light at $243 \mathrm{~nm}$ and $304 \mathrm{~nm}$ to $309 \mathrm{~nm}$ is used to drive twophoton transitions from either of the metastable 4D states to Rydberg S- or D-states (Chapter 4).

\section{Laser-driven transitions between electronic and motional states}

Spectra include carrier lines which are flanked by sidebands. A carrier line corresponds to a transition in which the electronic state is changed and the number of phonons is kept the same. When a sideband transition is excited the electronic state and the number of phonons are changed. Blue sidebands are more energetic and correspond to transitions in which the phonon number is increased, red sidebands are less energetic and correspond to transitions in which the 
phonon number is decreased. Phonon-number-changing transitions occur because of the momentum kick imparted to the trapped ion when a photon is absorbed. These transitions are shown in Fig. 3.2.
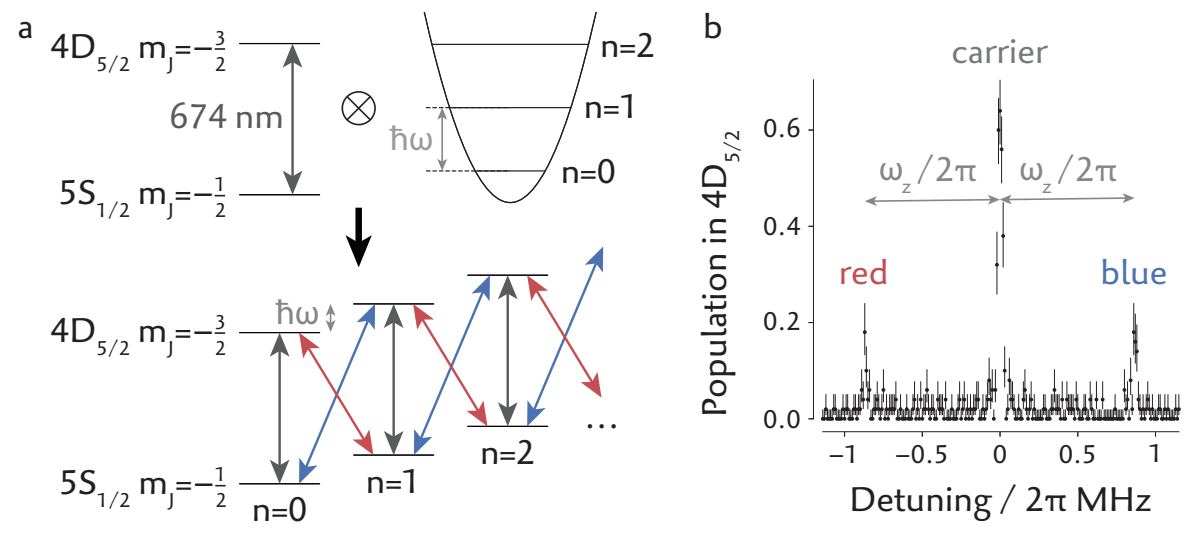

Figure 3.2: (a) Laser light drives transitions between electronic states and motional states. (b) Spectrum showing the $5 S_{1 / 2}, m_{J}=-\frac{1}{2} \leftrightarrow$ $4 D_{5 / 2}, m_{J}=-\frac{3}{2}$ carrier transition resonance and axial sidebands. The axial frequency is $\omega_{z}=2 \pi \times 867 \mathrm{kHz}$. Only firstorder sidebands are shown in (a) and (b). Error bars indicate quantum projection noise (68\% confidence interval).

The relative strength with which phonon-number-changing transitions are driven depends on the ratio of the recoil energy to the spacing of the quantum harmonic oscillator energy levels. This is described by the Lamb-Dicke parameter $\eta[87]$

$$
\begin{aligned}
\eta^{2} & =\left(\frac{\hbar^{2} k^{2} \cos ^{2} \phi}{2 M}\right) / \hbar \omega \\
& =\frac{\hbar k^{2} \cos ^{2} \phi}{2 M \omega} .
\end{aligned}
$$

Here $M$ is the ion mass, $\omega$ is the frequency of a particular motional mode, $k$ is the laser light wavevector and $\phi$ is the angle between the laser beam and the mode axis. When the recoil energy is much smaller than the quantised energy spacing, the Lamb-Dicke parameter is small and phonon-number-changing transitions are driven less strongly. The Lamb-Dicke parameter is different for different transitions, as well as different motional modes. 
The relative strength with which sideband transitions and carrier transitions are driven depends not only on the Lamb-Dicke parameter, but also on the number of phonons in a particular mode, $n .{ }^{1}$ The Lamb-Dicke regime is defined by the inequality

$$
\eta^{2}(2 n+1) \ll 1 .
$$

Within the Lamb-Dicke regime transitions which change the motional quantum number by more than one are strongly suppressed.

Working within the Lamb-Dicke regime is required for transitions to be driven coherently with high efficiency (since unwanted driving of sideband transitions causes dephasing), for resolved sideband cooling (which requires the phonon number to be usually preserved during spontaneous decay, see Section 3.1.2) and it is advantageous for high-precision spectroscopy (to avoid Doppler broadening of resonance lines). The Lamb-Dicke parameter for the axial motional mode and a $674 \mathrm{~nm}$ laser beam (used to drive the qubit transition) at $45^{\circ}$ to the trap axis is 0.05 when typical trapping parameters are used (for typical trapping parameters see Section 3.2.1). This laser beam is represented in Fig. 3.3(a). After Doppler cooling (Section 3.1.2) an ion has $\approx 16$ phonons in the axial mode and the Lamb-Dicke regime inequality [Eq. (3.2)] is satisfied for this particular laser beam and the axial motional mode.

Within the Lamb-Dicke regime the relative strengths with which carrier transitions and first-order sideband transitions are driven are given by [87]

$$
\begin{aligned}
\Omega_{n \rightarrow n} & =\left(1-\eta^{2} n\right) \Omega_{0}, \\
\Omega_{n \rightarrow n+1} & =\eta \sqrt{n+1} \Omega_{0}, \\
\Omega_{n \rightarrow n-1} & =\eta \sqrt{n} \Omega_{0},
\end{aligned}
$$

written to second order in $(\eta \sqrt{n}) ; \Omega_{0}$ is the Rabi frequency of carrier transitions when $n=0$. The ion temperature, that is the mean phonon number, is determined by comparing the Rabi frequencies with which sideband transitions are driven [87].

1 This $n$-dependence can be understood in terms of the $n$-dependence of the ladder operators $\hat{a}$ and $\hat{a}^{\dagger}$. 


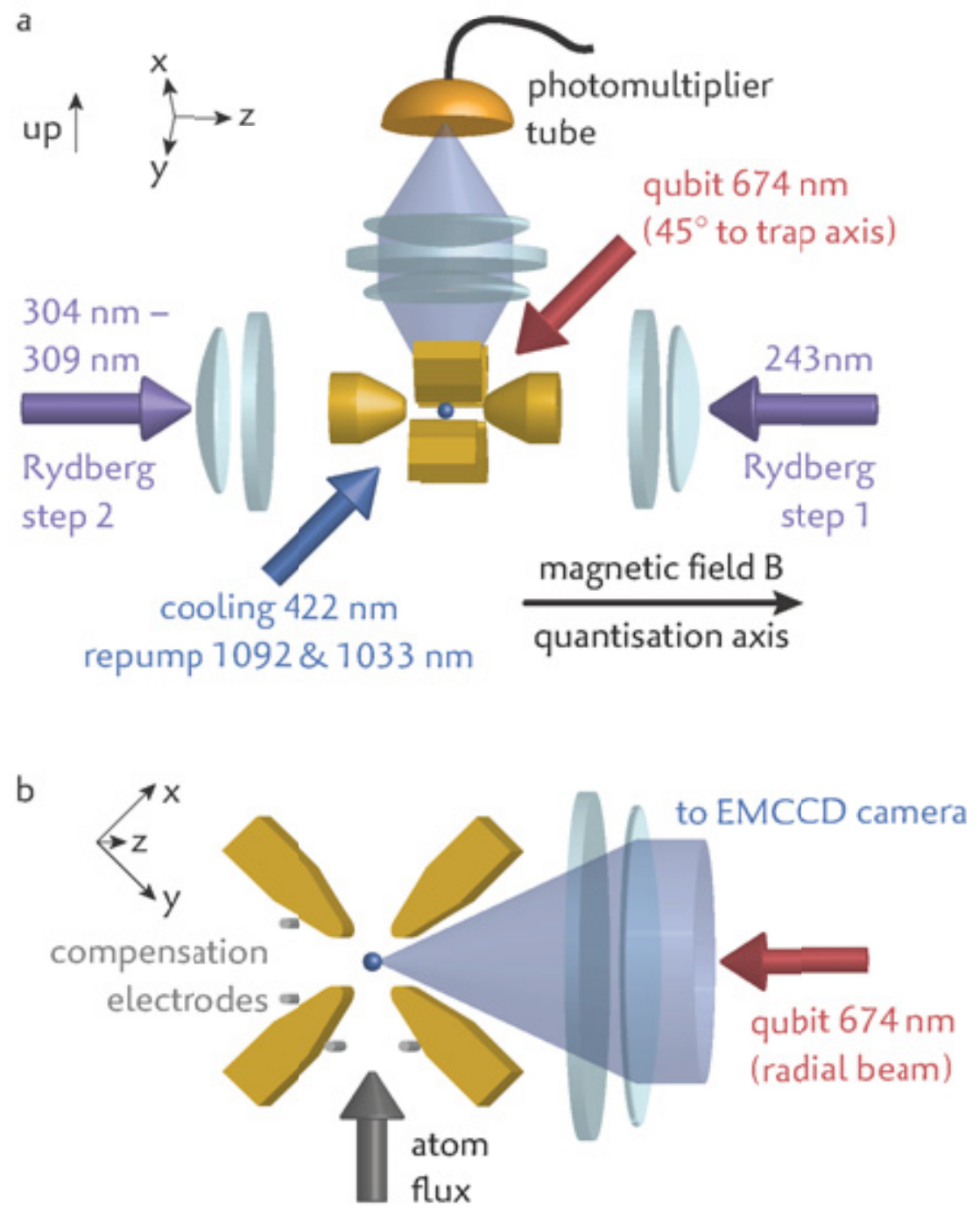

Figure 3.3: An ion is trapped between the linear Paul trap 'blade' and 'endcap' electrodes, which are gold-coated. Laser beams enter the vacuum chamber through viewports; the UV lasers pass through holes in the endcaps. Each micromotion compensation electrode consists of a pair of steel rods (shown in silver). Ion fluorescence is collected on the PMT and the EMCCD camera. Ions are loaded by resistivity-heating a Sr source: a flux of $\mathrm{Sr}$ atoms passes through the trap and lasers collinear with the $243 \mathrm{~nm}$ laser photo-ionise atoms. (a) Side view, (b) along the trap axis. 
Two-photon transitions between 4D states and Rydberg states are described by an effective Lamb-Dicke parameter, which is introduced in Section 4.4.

\subsubsection{Laser cooling}

At the beginning of each experiment run the ion is Doppler cooled. In many experiments resolved sideband cooling is also employed. Doppler cooling is used to cool an ion to the Lamb-Dicke regime ${ }^{2}$ while resolved sideband cooling is used to cool an ion below the Doppler cooling limit close to the motional ground state.

\section{Doppler cooling}

Doppler cooling proceeds as follows [87]: Due to the Doppler effect, the absorption coefficient of a laser-driven transition depends on the ion velocity. If a laser is appropriately detuned from the transition resonance, the radiation pressure force exerted by the laser light on the ion behaves as a drag force. The kinetic energy of the ion is then reduced and the ion is cooled.

In our lab the ion is Doppler cooled using $422 \mathrm{~nm}$ laser light reddetuned from the $5 \mathrm{~S}_{1 / 2} \leftrightarrow 5 \mathrm{P}_{1 / 2}$ resonance. The $422 \mathrm{~nm}$ laser beam k-vector has components along all three of the trap axes [Fig. 3.3(a)], this allows Doppler cooling in all directions with a single $422 \mathrm{~nm}$ laser beam [87]. After Doppler cooling the mean phonon number in the axial mode is $\bar{n}_{z} \approx 16$ and the mean phonon numbers in the radial modes are $\bar{n}_{x}, \bar{n}_{y} \approx 12$ when typical trapping frequencies are used. The mean phonon numbers dictate the temperatures of the motional modes, and they are determined as described in Section 3.1.1.

$1033 \mathrm{~nm}$ and $1092 \mathrm{~nm}$ laser light is used together with $422 \mathrm{~nm}$ laser light; $1092 \mathrm{~nm}$ laser light prevents optical pumping to $4 \mathrm{D}_{3 / 2}$, while $1033 \mathrm{~nm}$ laser light removes any ion population which was initially in $4 \mathrm{D}_{5 / 2}$. Doppler cooling is typically carried out for $1 \mathrm{~ms}$.

2 with regards the transitions driven in the experiment and the typical trapping frequencies used. 


\section{Resolved sideband cooling}

Resolved sideband cooling is a process in which ion population is optically pumped close to the motional ground state [87]. The process proceeds as follows (schematic in Fig. 3.4): The red sideband of a $5 S_{1 / 2} \leftrightarrow 4 D_{5 / 2}$ transition is driven; each excitation to $4 D_{5 / 2}$ reduces the phonon number by 1 . From $4 \mathrm{D}_{5 / 2}$ population may be driven back to $5 S_{1 / 2}$ (and the lost phonon is regained) or population may decay to $5 S_{1 / 2}$. Within the Lamb-Dicke regime the decay usually preserves the phonon number and thus on average the phonon number is reduced during the process. The process is sped up by coupling $4 \mathrm{D}_{5 / 2}$ and $5 \mathrm{P}_{3 / 2}$ such that the effective lifetime of $4 \mathrm{D}_{5 / 2}$ is reduced.

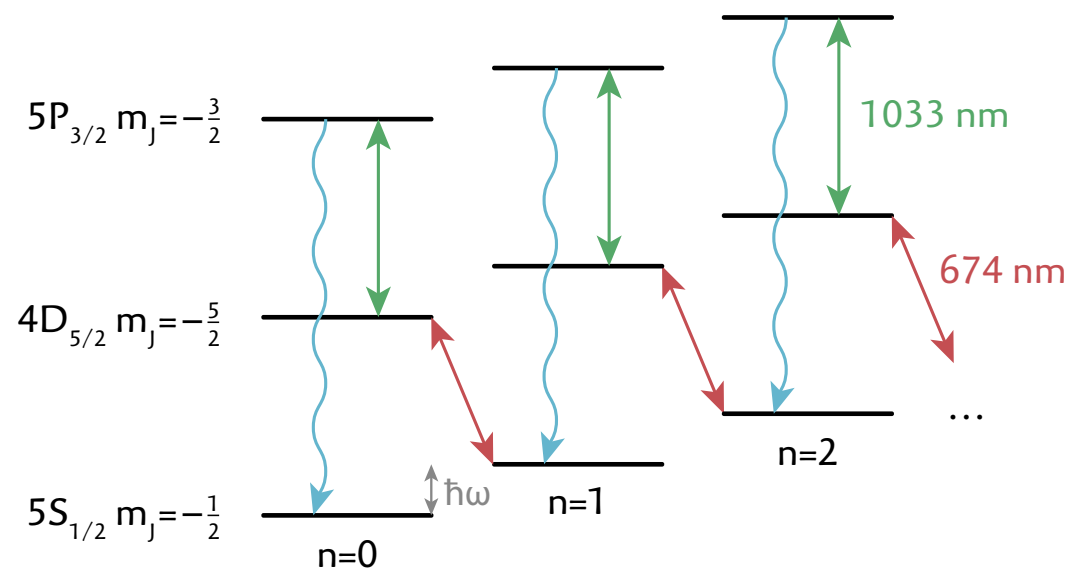

Figure 3.4: During sideband cooling each excitation from $5 S_{1 / 2}$ to $4 D_{5 / 2}$ reduces the phonon number $(n)$ by 1 , and decay to $5 S_{1 / 2}$ usually preserves the phonon number (within the Lamb-Dicke regime). The effective lifetime of the excited state is shortened by coupling $4 D_{5 / 2}$ and $5 P_{3 / 2}$. The energy spacing of the motional levels is $\hbar \omega$.

The radial $674 \mathrm{~nm}$ laser beam in Fig. 3.3 is used to cool radial motional modes, the angled beam is used to cool the axial motional mode. Typically sideband cooling is carried out for $1 \mathrm{~ms}$ for the axial mode and for $1 \mathrm{~ms}$ for the two radial modes. After sideband cooling the mean phonon numbers in the radial modes and in the axial mode are $\bar{n}_{x}, \bar{n}_{y}, \bar{n}_{z} \approx 0.2$ when typical trapping frequencies are used. 


\subsubsection{State initialisation}

Different experiments require the ion to be initialised in different states. The initialisation procedures are described in this section.

Initialisation in $|1\rangle \equiv 5 S_{1 / 2}, m_{J}=-\frac{1}{2}$ by frequency-resolved optical pumping

There are two $5 S_{1 / 2}$ states, labelled by $m_{J}= \pm \frac{1}{2}$. State $5 S_{1 / 2}, m_{J}=$ $-\frac{1}{2} \equiv|1\rangle$ is used together with $4 D_{5 / 2}, m_{J}=-\frac{5}{2} \equiv|0\rangle$ to store a qubit. Initialisation in $|1\rangle$ proceeds as follows: First, any ion population residing in the metastable $4 \mathrm{D}$ states is removed and sent to $5 S_{1 / 2}$ via the 5P states using $1033 \mathrm{~nm}$ and $1092 \mathrm{~nm}$ laser light. Then any ion population residing in $5 S_{1 / 2}, m_{J}=+\frac{1}{2}$ is optically pumped to qubit state $|1\rangle \equiv 5 S_{1 / 2}, m_{J}=-\frac{1}{2}$ by driving the following cycle $\sim 10$ times:

1. A $\pi$ pulse is applied on the frequency-resolved $5 S_{1 / 2}, m_{J}=$ $+\frac{1}{2} \leftrightarrow 4 \mathrm{D}_{5 / 2}, \mathrm{~m}_{\mathrm{J}}=-\frac{3}{2}$ transition. Any population residing in $|1\rangle$ is unaffected, while population in $5 S_{1 / 2}, m_{J}=+\frac{1}{2}$ is excited to $4 D_{5 / 2}, m_{J}=-\frac{3}{2}$.

2. Population is removed from the $4 \mathrm{D}$ states and returned to $5 S_{1 / 2}$ via the $5 \mathrm{P}$ states using $1033 \mathrm{~nm}$ and $1092 \mathrm{~nm}$ laser light.

Initialisation in $|0\rangle \equiv 4 \mathrm{D}_{5 / 2}, \mathrm{~m}_{\mathrm{J}}=-\frac{5}{2}$

The ion is prepared in $|1\rangle$ by optical pumping and then excited to qubit state $|0\rangle \equiv 4 \mathrm{D}_{5 / 2}, \mathrm{~m}_{\mathrm{J}}=-\frac{5}{2}$ by applying a $\pi$ pulse on the $674 \mathrm{~nm}$ $|1\rangle \leftrightarrow|0\rangle$ transition. Electron shelving (Section 3.1.4) is then used to confirm excitation to $|0\rangle$; cases in which initialisation is unsuccessful are removed during data analysis.

Initialisation in $|1\rangle+e^{i \phi}|0\rangle$

The ion is prepared in $|1\rangle$ by optical pumping and then the superposition $|1\rangle+e^{i \phi}|0\rangle$ is excited by driving a $\frac{\pi}{2}$ pulse on the $674 \mathrm{~nm}|1\rangle \leftrightarrow|0\rangle$ transition with laser phase $\phi+\frac{\pi}{2}$. 


\section{Initialisation in a phonon number state}

The ion is prepared in a state with $n_{x}$ and $n_{y}$ radial phonons in the $x$ and $y$ radial modes as follows:

1. The radial motional modes are sideband cooled close to their ground states.

2. The ion is prepared in $|1\rangle$ by optical pumping.

3. The following cycle is carried out $n_{x}$ times:

a) A $\pi$ pulse is applied on the $x$-motional mode blue sideband of the $|1\rangle \leftrightarrow|0\rangle$ transition. The pulse length accounts for the phonon-number-dependent Rabi frequency described by Eq. (3.3).

b) Electron shelving is used to confirm excitation to $|0\rangle$.

c) A $\pi$ pulse is applied on the $|1\rangle \leftrightarrow|0\rangle$ carrier transition to return population to $|1\rangle$.

d) $1033 \mathrm{~nm}$ and $1092 \mathrm{~nm}$ laser light is used to remove any residual population from the 4D states.

4. The cycle is repeated $n_{y}$ times, this time the $y$-motional mode blue sideband is driven.

5. In some experiments the ion is then transferred to $|0\rangle$ by driving a $\pi$ pulse on the $|1\rangle \leftrightarrow|0\rangle$ transition.

This method allows us to prepare an ion with 40 radial phonons with $\approx 20 \%$ efficiency. We check the initialisation by preparing an ion in $|1\rangle$ with $n_{y}$ phonons in the y-mode and then driving Rabi oscillations on the y-motional mode blue sideband of the $|1\rangle \leftrightarrow|0\rangle$ transition, results are shown in Fig. 3.5. The Rabi frequency scales as $\sqrt{n_{y}+1}$, as expected from Eq. (3.3).

\section{Initialisation in $4 \mathrm{D}_{3 / 2}$}

Population is optically pumped from $5 S_{1 / 2}$ to $4 \mathrm{D}_{3 / 2}$ by driving the $422 \mathrm{~nm} 5 \mathrm{~S}_{1 / 2} \leftrightarrow 5 \mathrm{P}_{1 / 2}$ transition; from $5 \mathrm{P}_{1 / 2}$ the ion decays to $4 \mathrm{D}_{3 / 2}$ in $6 \%$ of the cases [86]. A mixture of $4 \mathrm{D}_{3 / 2}$ Zeeman sublevels is populated. 
a

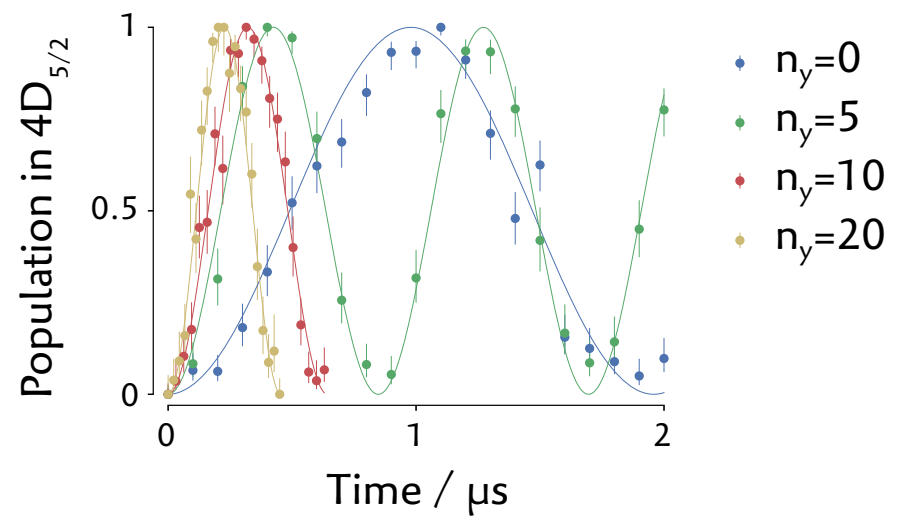

b

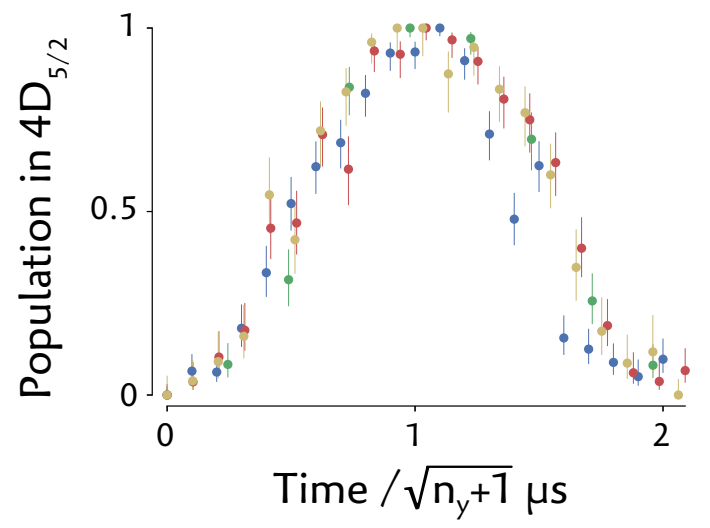

- $\mathrm{n}_{\mathrm{y}}=0$

- $n_{y}=5$

- $\mathrm{n}_{\mathrm{y}}=10$

$n_{y}=20$

Figure 3.5: Preparation of phonon number states is demonstrated by driving high-contrast Rabi oscillations on a $5 S_{1 / 2} \leftrightarrow 4 \mathrm{D}_{5 / 2}$ blue motional sideband transition and observing the $\sqrt{n_{y}+1}$-scaling of the Rabi frequency with the phonon number $n_{y}$ in the $y$ radial mode. (a) The horizontal axis is the excitation time, (b) the time axis is scaled to make the $\sqrt{n_{y}+1}$-dependence clear. Sinusoidal fit functions in (a) serve to guide the eye. Error bars indicate quantum projection noise ( $68 \%$ confidence interval).

\subsubsection{Measurement}

Measurements involve a technique called electron shelving. Electron shelving distinguishes an ion in state $4 \mathrm{D}_{5 / 2}$ from an ion in states $5 \mathrm{~S}_{1 / 2}$ or $4 \mathrm{D}_{3 / 2}$, as described below. 
In terms of a qubit stored in $5 \mathrm{~S}_{1 / 2}$ and $4 \mathrm{D}_{5 / 2}$, electron shelving is a projective measurement in the $\hat{\sigma}_{z}$ basis. By driving a $\frac{\pi}{2}$ pulse on the $|1\rangle \leftrightarrow|0\rangle$ transition with laser phase $0\left(\frac{\pi}{2}\right)$ before carrying out electron shelving, a projective measurement in the $-\hat{\sigma}_{y}\left(-\hat{\sigma}_{x}\right)$ basis is carried out. Measurements in different bases allow us to carry out quantum state tomography and quantum process tomography, these techniques are described in Section 7:4.3.

To distinguish population in $5 \mathrm{~S}_{1 / 2}$ and $4 \mathrm{D}_{3 / 2}$ we first transfer population from the $5 S_{1 / 2}$ sublevels to $4 D_{5 / 2}$ sublevels by driving $674 \mathrm{~nm}$ transitions and then we use electron shelving.

\section{Electron shelving}

The ion is illuminated by $422 \mathrm{~nm}$ and $1092 \mathrm{~nm}$ laser light and we look for scattered $422 \mathrm{~nm}$ photons using a PMT. When the PMT detects a photon it sends a digital signal to a counter, which is connected to a PC (Section 3.4). If the ion was initially in $5 S_{1 / 2}$ or $4 D_{3 / 2} 422 \mathrm{~nm}$ photons are scattered by the ion, whereas if the ion is in state $4 D_{5 / 2}$ no light is scattered by the ion and the PMT shows only a background signal due to background light and PMT dark counts. Measuring for $500 \mu \mathrm{s}$ allows us to distinguish between an ion in state $4 \mathrm{D}_{5 / 2}$ and an ion in state $5 S_{1 / 2}$ or $4 D_{3 / 2}$ with $\sim 99.9 \%$ efficiency, as shown in Fig. 3.6. The $500 \mu \mathrm{s}$ detection time greatly exceeds the lifetimes of all the atomic states except for the metastable 4D states (lifetimes $\sim 400 \mathrm{~ms}[82,84])$ and the ground state $5 \mathrm{~S}_{1 / 2}$.

The states of multiple ions are measured by imaging the ions on a camera and using the electron shelving technique. However, most of the measurements in this thesis are concerned with a single ion.

\subsection{LINEAR PAUL TRAP}

In this experiment ions are trapped in a macroscopic linear Paul trap in an ultra-high vacuum $\left(<10^{-10} \mathrm{mbar}\right)$. In this section key features of the trap are presented. Information about the assembly of the trap and the vacuum chamber may be found in the master's thesis of Fabian Pokorny [88]. 


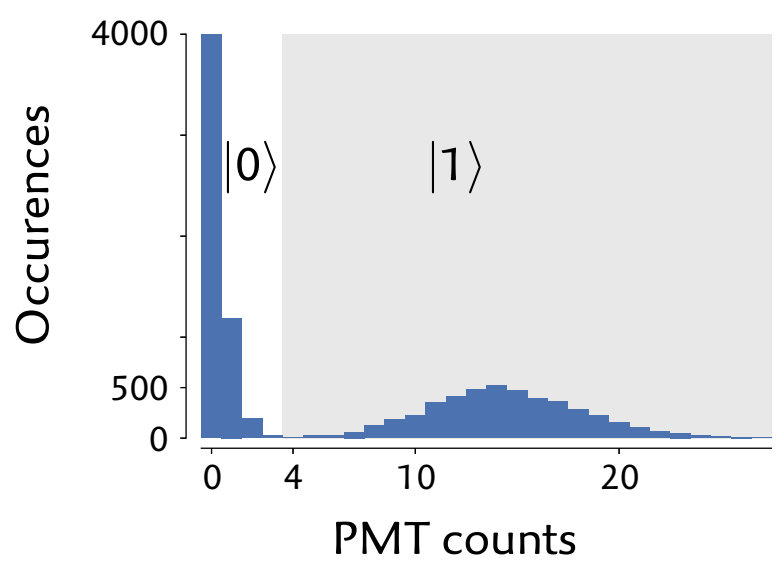

Figure 3.6: Electron shelving using photon counts measured with the PMT. If 4 or more counts are recorded during the $500 \mu$ s measurement time the ion is most probably in state $5 S_{1 / 2}$ (qubit state $|1\rangle$ ). If 3 or fewer counts are recorded the ion is likely in state $4 D_{5 / 2}$

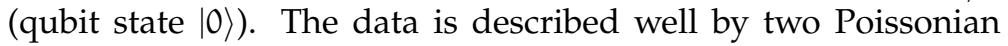
distributions with mean values 0.3 and 14 , the overlap of the distributions is $0.04 \%$. This overlap as well as decay from $4 D_{5 / 2}$ during the measurement time cause errors $\sim 0.1 \%$.

\subsubsection{Ions in the trapping potential}

The linear Paul trap uses a combination of static and oscillating electric quadrupole fields which result in effective harmonic trapping potentials in the three spatial dimensions, as described in Section 6.1.1. The oscillating field is driven at $\Omega_{\mathrm{rf}}=2 \pi \times 18.2 \mathrm{MHz}$. Typically the oscillating field has gradient $\alpha \approx 8.4 \times 10^{8} \mathrm{~V} \mathrm{~m}^{-2}$ and the static field has gradient $\beta \approx 6.8 \times 10^{6} \mathrm{~V} \mathrm{~m}^{-2}$. Trapping frequencies $\omega_{x, y} \approx$ $2 \pi \times 1.7 \mathrm{MHz}$ and $\omega_{z} \approx 2 \pi \times 840 \mathrm{kHz}$ result, where $x$ and $y$ are the radial directions and $z$ is the axial direction of the trap (see Fig. 3.3). The confinement is weaker along the trap axis such that multiple trapped ions form a string; the mutual repulsion between the ions keeps them apart by $\approx 5 \mu \mathrm{m}$. 
Ion fluorescence is collected by a $\mathrm{PMT}^{3}$ and it is also used to image individual ions on an EMCCD camera ${ }^{4}$, as shown in Fig. 3.7.

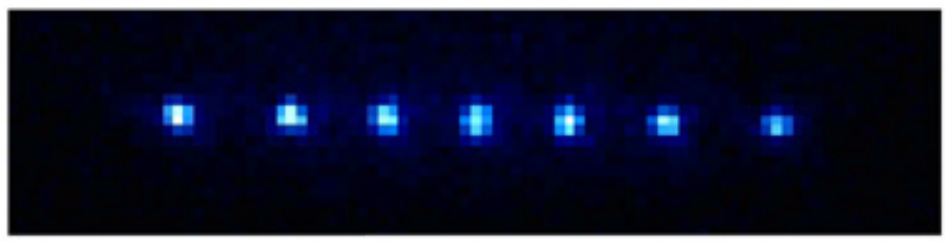

Figure 3.7: String of seven ${ }^{88} \mathrm{Sr}^{+}$ions imaged on the EMCCD camera.

\subsubsection{Trap components}

The trap parts were fabricated at the Institute for Quantum Optics and Quantum Information (IQOQI), Innsbruck. The linear Paul trap consists of titanium electrodes mounted in a sapphire holder, which itself is in a titanium mount. The electrodes are coated in gold. A trapped ion is separated from the blade electrodes by $500 \mu \mathrm{m}$. Holes in the endcap electrodes provide optical access along the trap axis with numerical aperture 0.058 . This design builds upon previous macroscopic linear Paul traps machined in Innsbruck.

\subsubsection{Trap temperature}

In a trap of the same design it was found that the typical powers used for driving the oscillating trapping field $(\approx 1 \mathrm{~W})$ increase the trap temperature by $\approx 1.4^{\circ} \mathrm{C}[89]$. Temperature increases two orders of magnitude higher have been recorded elsewhere [90] resulting in increased blackbody radiation which causes shifts in atomic clock transitions. In our experiment increased blackbody radiation would decrease Rydberg state lifetimes (see Section 2.4).

The temperature change measured in the trap of the same design as ours is relatively small because of the high thermal conductivity of the trap components and the high electrical conductivity of the gold-

3 Hamamatsu Photonics H10682-210

4 Andor iXon3 897 
coated electrodes. We use similar powers for driving the oscillating trapping field in our experiment and we expect similar temperature changes $\left(\sim 1.4^{\circ} \mathrm{C}\right)$.

\subsubsection{Photo electrons induced by UV light}

The gold coating on the electrodes also means photo electrons are unlikely to be produced by stray UV laser light illuminating an electrode surface, owing to the high work function of gold $>5.1 \mathrm{eV}$ [91], cf. $243 \mathrm{~nm}$ photons carry $5.1 \mathrm{eV}$ energy.

Photo electrons cause the build-up of stray charges inside the chamber which disturb the trapping potential (Section 3.2.8), additionally a flux of photo electrons may perturb highly-sensitive Rydberg states [75].

In one of the traps used in the Rydberg ion experiment in Mainz stray charges were generated by VUV laser light hitting surfaces; these charges disturbed the trapping potential and this contributed to their broad Rydberg resonance linewidths [73] (see Section 1.3.2).

\subsubsection{Motional heating}

Motional heating is detrimental to quantum manipulation of trapped ions [17] and is likely harmful in trapped Rydberg ions systems since the energy for Rydberg excitation depends on the number of phonons in the system (Section 6.1). Furthermore motional heating is itself a symptom of electric field noise which may perturb Rydberg ions directly.

The upper bounds of heating rates in our trap are relatively low; in the axial mode the rate is $<18$ phonon $\mathrm{s}^{-1}$ and in the radial modes the rate is $<4$ phonon $\mathrm{s}^{-1}$ when $\omega_{x, y} \approx 2 \pi \times 1.7 \mathrm{MHz}$ and $\omega_{z}=2 \pi \times$ $870 \mathrm{kHz}$. The heating rate is inferred by first cooling the ion and then measuring the ion temperature (Section 3.1.1) after different time delays, the same method is used in [92]. The heating rate is not precisely determined because servo bumps of the qubit laser drive off-resonant transitions making temperature measurements difficult for phonon numbers $<\sim 0.2$. 


\subsubsection{Magnetic field}

Coils outside the chamber apply a magnetic field along the trap axis with strength $\sim 0.3 \mathrm{mT}$ at the position of trapped ions. Since the magnetic quantum number is a good quantum number in our system (for low-lying states and Rydberg S-states), the field defines the quantisation axis. The magnetic field is aligned along the trap axis as follows: A beam of circularly-polarised $422 \mathrm{~nm}$ laser light is sent along the trap axis through holes in the endcap electrodes. When the magnetic field direction is collinear with the laser beam propagation the $422 \mathrm{~nm}$ laser light drives only one $\sigma$-transition between $5 \mathrm{~S}_{1 / 2}$ and $5 \mathrm{P}_{1 / 2}$ sublevels, and a sublevel of $5 S_{1 / 2}$ becomes a dark state. Currents in the coils are varied until a dark state appears and the ion fluorescence is minimised.

\subsubsection{Ion loading}

In our experiment a trapped ${ }^{88} \mathrm{Sr}^{+}$ion is lost by double ionisation after typically several hundred excitations to a Rydberg state (see Chapter 5). Double ionisation occurs once in every 2300 excitations to a Rydberg state in the Mainz experiment [75]. Rydberg ion experiments thus require fast and reliable ion loading.

Until recently, and for most of the experiments in this thesis, single ${ }^{88} \mathrm{Sr}^{+}$ions were loaded by resistively heating a strontium oven to produce a beam of strontium atoms which passes through the trap, atoms were then ionised in a two-step photoionisation process [93] and the ions were Doppler cooled (see Section 3.1.2). Around 10 minutes were spent loading a ${ }^{88} \mathrm{Sr}^{+}$ion. After loading we let the oven cool for an additional 10 minutes before carrying out Rydberg experiments, since we suspect blackbody radiation increases the likelihood of losing a Rydberg ion by double-ionisation.

Recently laser ablation loading was introduced to our system and now ions are loaded in less than 30 seconds without the system being heated. 


\subsubsection{Micromotion compensation}

In a linear Paul trap ions are confined in a combination of oscillating and static electric quadrupole fields. Ideally the nulls of the electric quadrupole fields overlap and the ion is trapped at the null where the electric field strength is zero. Imperfections in the construction of the trap and stray charges (Section 3.2.4) cause the nulls of the quadrupole fields to be at different positions. This results in oscillating and static electric dipole fields at the average position of the ion which drive motion called 'excess micromotion' [94, 95].

Excess micromotion alters atomic transition lineshapes, disrupts laser cooling and shifts resonance frequencies [94, 95]. In quantum information processing experiments and atomic clock experiments steps are taken to minimise excess micromotion. Rydberg ions have extremely large polarisabilities; electric fields cause Rydberg resonance lineshapes to be altered (see Section 6.1) and they can also cause field ionisation [49]. Minimisation of micromotion and thus the electric fields experienced by the Rydberg ion is important in our experiment.

Our linear Paul trap has electrodes for minimisation of radial micromotion [see Fig. 3.3(b)]. By applying appropriate static voltages to these electrodes the position of the null of the static electric quadrupole field is made to overlap with the null of the oscillating electric quadrupole field.

We find appropriate voltages for minimisation of micromotion using established techniques [94, 95]; we monitor the displacement of a trapped ion as the trap depth is changed by imaging the ion on the EMCCD camera, we use the cross-correlation technique, and we employ the resolved sideband method using the $5 \mathrm{~S}_{1 / 2} \leftrightarrow 4 \mathrm{D}_{5 / 2}$ transition. After minimising micromotion using the resolved sideband method the typical strength of the residual oscillating field at the position of the ion is $\sim 10 \mathrm{~V} \mathrm{~m}^{-1}$ when typical trapping frequencies are used.

In Section 6.1.2 we investigate the effects of non-overlapping electric quadrupole trapping fields on a Rydberg ion and introduce a novel method for minimising micromotion using a trapped Rydberg ion. 


\section{$3 \cdot 3$ LASER SYSTEMS}

Laser systems are described in this section. Laser beams pass from the laser sources (Section 3.3.1), through acousto-optic modulators (AOMs) used for laser pulse shaping (Section 3.3.2) before they are brought close the to the experiment chamber via single-mode optical fibres (Section 3.3.3). Laser pulses then enter the chamber through viewports (Fig. 3.3) and are focussed onto trapped ions.

So that the transitions in Fig. 3.1 may be driven efficiently laser frequencies are stabilised by using optical resonators as frequency references (Section 3.3.4). A schematic of a typical beam path is shown in Fig. 3.8.

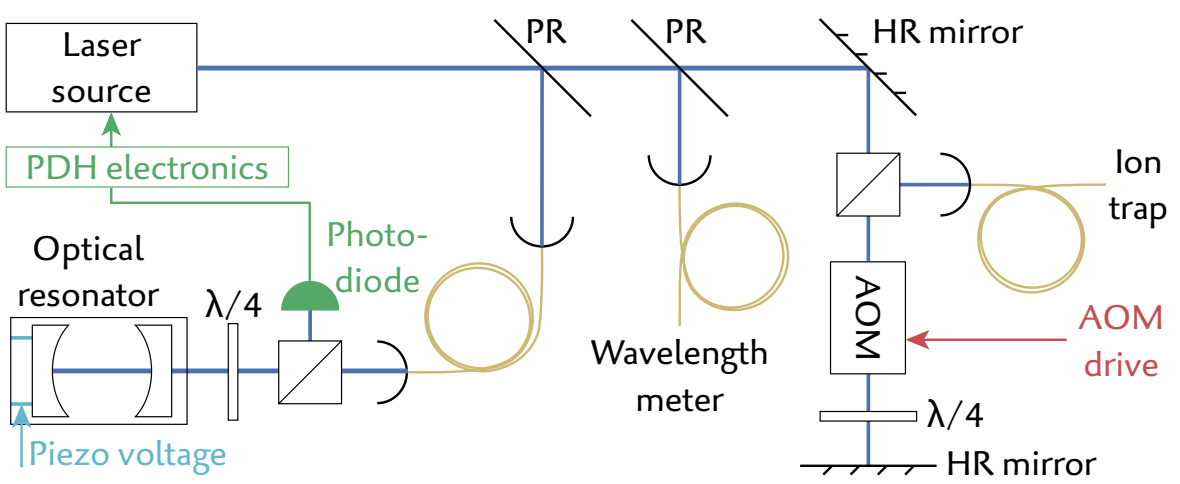

Figure 3.8: Schematic of a typical laser setup. Highly-reflective and partiallyreflective mirrors are labelled HR and PR. Wavelength meter HighFinesse WS6-200 is used.

\subsubsection{Laser sources}

Laser light for driving the transitions between low-lying states is common to other ${ }^{88} \mathrm{Sr}^{+}$experiments which study quantum computing [96-98] and atomic clocks [99, 10o]. UV laser light for Rydberg excitation is specific to our experiment. 
Laser light for transitions between low-lying states

We use diode lasers 5 to produce $422 \mathrm{~nm}, 1033 \mathrm{~nm}$ and $1092 \mathrm{~nm}$ laser light and a diode-laser-pumped tapered-amplifier system to produce 674 nm laser light. ${ }^{6}$

\section{Laser light for the first Rydberg-excitation step}

Laser light at $243 \mathrm{~nm}$ for the first Rydberg-excitation step is produced in a commercial system, 7 as follows: Laser light at $970 \mathrm{~nm}$ is produced by a diode-laser-pumped tapered-amplifier system. Two cascaded second harmonic generation (SHG) stages are used to upconvert the $970 \mathrm{~nm}$ fundamental to $243 \mathrm{~nm}$ laser light. Each SHG stage consists of a nonlinear crystal in a bow-tie cavity. Crystals of lithium triborate (LBO) and barium metaborate (BBO) are used in the first and second stages respectively. Similar laser systems are used for spectroscopy of atomic hydrogen [101].

\section{Laser light for the second Rydberg-excitation step}

Tunable laser light between $304 \mathrm{~nm}$ and $309 \mathrm{~nm}$ is required for the second Rydberg-excitation step. The tunability allows a range of states to be excited, from principal quantum number $n=24$ up to the second ionisation threshold. This laser light is produced in two steps:

5 Toptica DL pro

6 Toptica TA pro; a high-power source is used because the $674 \mathrm{~nm} 5 \mathrm{~S}_{1 / 2} \leftrightarrow 4 \mathrm{D}_{5 / 2}$ transition is electric-dipole-forbidden and relatively high laser light intensities are required to drive it.

7 Toptica TA-FHG pro 
First $1551 \mathrm{~nm}$ laser light from a diode-laser ${ }^{8}$ fibre-amplifier ${ }^{9}$ system and tunable laser light between $1000 \mathrm{~nm}$ and $1030 \mathrm{~nm}$ from a diodelaser-pumped tapered-amplifier system ${ }^{10}$ pass through a periodicallypoled lithium niobate (PPLN) crystal $^{11}$ and tunable laser light in the range $608 \mathrm{~nm}$ to $618 \mathrm{~nm}$ is produced by sum frequency generation (SFG). To achieve quasi-phase matching across the entire $608 \mathrm{~nm}$ to $618 \mathrm{~nm}$ range the crystal temperature is varied and crystal channels with different poling periodicities are used. This first step was the master's thesis work of Christine Maier [102].

In the second step $608 \mathrm{~nm}$ to $618 \mathrm{~nm}$ laser light is upconverted to $304 \mathrm{~nm}$ to $309 \mathrm{~nm}$ laser light in a commercial system consisting of a LBO crystal in a bow-tie cavity, ${ }^{12}$ here angle-phase matching is used. Similar laser systems are used in experiments with trapped ${ }^{9} \mathrm{Be}^{+}$ions $[103,104]$.

\section{Laser light for photoionisation of ${ }^{88} \mathrm{Sr}$ atoms}

Ion loading (Section 3.2.7) involves two-step photoionisation of neutral ${ }^{88} \mathrm{Sr}$ atoms using laser light produced by diode lasers at $405 \mathrm{~nm}^{13}$ and $461 \mathrm{~nm} \cdot{ }^{14}$

8 Originally a TeraXion PS-NLL DFB semiconductor laser was used. The power spectrum consists of a Lorentzian line superposed onto a broad envelope. While $80 \%$ of the laser power lies within $2 \pi \times 12 \mathrm{kHz}$ of the centre frequency, $8 \%$ of the laser power lies outside $2 \pi \times 1 \mathrm{MHz}$ of the centre frequency (using an observation time of $1 \mathrm{~ms}$ ). The broad envelope does not allow stable locking of the $608 \mathrm{~nm}$ to $618 \mathrm{~nm}$ laser light to the reference cavity, and it inhibits coherent excitation of Rydberg states using $304 \mathrm{~nm}$ to $309 \mathrm{~nm}$ laser light. We now use a NKT Koheras BASIK E15 DFB fibre laser which has a narrow power spectrum and allows stable locking of the $608 \mathrm{~nm}$ to $618 \mathrm{~nm}$ laser light.

9 Manlight EYFA-CW-SLM-P-TKS

10 Toptica TA pro

11 Covesion MSFG612-0.5-40

12 Toptica SHG pro

13 InsaneWare blu-ray diode used originally, recently replaced by a fibre-coupled system Thorlabs LP405-SFio.

14 Toptica DL pro 


\subsubsection{Laser pulse generation using AOMs}

Most of the lasers setups include AOMs in double-pass configurations [105]. Laser pulses are engineered by controlling the amplitude, frequency and phase of radiofrequency AOM drive signals (Section 3.4). Laser pulses can be produced with constant phase relations between them. The time taken for sound waves in an AOM crystal to move across a laser beam limits the minimum pulse length to $\sim 50 \mathrm{~ns}$.

\subsubsection{Optical fibres}

Single-mode fibres allow stable beam pointing onto the ions and they also clean up the laser beams. Polarisation-maintaining single-mode step-index fibres transmit $405 \mathrm{~nm}, 422 \mathrm{~nm}, 461 \mathrm{~nm}, 674 \mathrm{~nm}, 1033 \mathrm{~nm}$ and $1092 \mathrm{~nm}$ laser light close to the experiment chamber. Before focussing onto the ions $422 \mathrm{~nm}, 1033 \mathrm{~nm}$ and $1092 \mathrm{~nm}$ laser light is overlapped in an endlessly single-mode photonic crystal fibre. ${ }^{15}$

\section{Single-mode fibres for UV laser light}

Conventional single-mode step-index fibres made of silicon dioxide are unsuitable for transmitting UV laser light; they degrade due to ultraviolet solarisation. We use hydrogen-loaded photonic crystal fibres with relatively large mode field diameters $\sim 8 \mu \mathrm{m}$ which resist UV solarisation [106]. ${ }^{15}$ Details can be found in the master's thesis of Johannes Haag [107].

Typically up to $3 \mathrm{~mW}$ of $243 \mathrm{~nm}$ laser light and $50 \mathrm{~mW}$ of $304 \mathrm{~nm}$ to $309 \mathrm{~nm}$ laser light is focussed onto the ions. Techniques developed for focussing these laser beams onto trapped ions are described in Section 4.2.

\subsubsection{Laser frequency stabilisation}

To efficiently drive the transitions in Fig. 3.1 lasers must be resonant with the transitions and their linewidths must be lower than the cor- 
responding transition linewidths. Most of the diode lasers have freerunning linewidths $\sim 2 \pi \times 200 \mathrm{kHz}$. The lasers' linewidths are reduced and their centre frequencies are controlled by using optical resonators as frequency references. The Pound-Drever-Hall (PDH) technique $[108,109]$ is used to confer the stability of the lengths of optical resonators onto the frequency stability of lasers.

Most of the lasers are stabilised using optical resonators built inhouse. The $674 \mathrm{~nm}$ laser is locked to a highly-stable commercial optical resonator which has a high finesse.

\section{Stabilisation using optical resonators built in-house}

Eight optical resonators with stable lengths were built following the design in [110]. A clear schematic is in [111].

In each optical resonator setup one of the mirrors is mounted on piezoelectric rings; by changing the voltages applied to the piezo rings the length of each resonator is controlled. Each resonator is used to stabilise the frequency of one laser. Because the length of each resonator can be changed by more than half of the laser wavelength, the stabilised lasers can be tuned to any frequency.

The eight optical resonators were designed for eight different wavelengths of laser light: $422 \mathrm{~nm}, 608 \mathrm{~nm}$ to $618 \mathrm{~nm}, 674 \mathrm{~nm}, 970 \mathrm{~nm}$, $1000 \mathrm{~nm}$ to $1030 \mathrm{~nm}, 1033 \mathrm{~nm}, 1092 \mathrm{~nm}$ and $1550 \mathrm{~nm}$. The mirror coatings of each of the resonators were chosen for a particular laser wavelength.

The $422 \mathrm{~nm}, 1033 \mathrm{~nm}$ and $1092 \mathrm{~nm}$ lasers are stabilised to optical resonators with finesses $\sim 1000$ and the stabilised laser linewidths are $\sim 2 \pi \times 100 \mathrm{kHz}$. The $608 \mathrm{~nm}$ to $618 \mathrm{~nm}$ and $970 \mathrm{~nm}$ laser light is stabilised to optical resonators with finesses $\sim 15000$. The $608 \mathrm{~nm}$ to $618 \mathrm{~nm}$ laser light is locked by sending feedback to the $1000 \mathrm{~nm}$ to $1030 \mathrm{~nm}$ fundamental laser. The frequency stability of the fundamental lasers is conferred onto the Rydberg-excitation laser light; this results in laser light with linewidth $\sim 2 \pi \times 100 \mathrm{kHz}$ at both $243 \mathrm{~nm}$ and $304 \mathrm{~nm}$ to $309 \mathrm{~nm}$.

Optical resonators for $1000 \mathrm{~nm}$ to $1030 \mathrm{~nm}$ and $1550 \mathrm{~nm}$ laser light are used for diagnostic purposes. An optical resonator for $674 \mathrm{~nm}$ laser light was set up. It has finesse $\sim 10000$ and it may be used in the 
future to implement a two-stage laser lock [111, 112] together with the commercial high-finesse optical resonator described below.

The stabilised laser linewidths are estimated by an in-loop method: the gradient of the PDH error signal is used to convert the standard deviation of the error signal of a locked laser to a laser linewidth.

Sidebands are required for laser frequency stabilisation by the PDH technique. Sidebands are introduced by modulating the laser diode current of the $422 \mathrm{~nm}, 970 \mathrm{~nm}, 1000 \mathrm{~nm}$ to $1030 \mathrm{~nm}, 1033 \mathrm{~nm}$ and $1092 \mathrm{~nm}$ lasers. The sidebands persist in the laser light produced by SFG and SHG, and they are used to stabilise the lengths of the SHG bow-tie cavities to the laser frequencies by employing the PDH technique.

\section{Stabilisation using the high-finesse resonator}

For coherent operations between the qubit states (sublevels of $5 S_{1 / 2}$ and $4 \mathrm{D}_{5 / 2}$ ) it is necessary to have a highly-stable $674 \mathrm{~nm}$ laser. Using a commercial optical resonator ${ }^{16}$ with a high-finesse $\sim 100000$ the laser frequency was stabilised to $\sim 2 \pi \times 1 \mathrm{kHz}$ during the master's thesis work of Florian Kress [113].

Because sidebands in the laser light would disrupt coherent manipulation of the ion, we introduce sidebands to the $674 \mathrm{~nm}$ laser using an electro-optic modulator immediately before the optical resonator. The length of the high-finesse cavity cannot be scanned; to make up for the $\approx 2 \pi \times 1.41 \mathrm{GHz}$ mismatch between the high-finesse cavity resonance and the $5 S_{1 / 2} \leftrightarrow 4 \mathrm{D}_{5 / 2}$ atomic resonance multiple AOMs are used.

Frequency drifts of the high-finesse resonator are countered by using the ion as a frequency reference in a Ramsey-type scheme described in [114, 115]. This method also allows fluctuations in the magnetic field strength at the position of the ion to be compensated such that transitions between different Zeeman sublevels of $5 \mathrm{~S}_{1 / 2}$ and $4 \mathrm{D}_{5 / 2}$ may be resonantly driven. 


\subsection{ELECTRONICS FOR EXPERIMENTAL CONTROL}

A PC controls two electronic systems which are used for running experiments: the pulse sequencer box and a bus system. The pulse sequencer box controls the timing of each experiment run. It outputs digital signals and RF signals with a timing resolution of $10 \mathrm{~ns}$. It is described in detail in [116, 117]. The bus system also controls RF signals, though its outputs are updated over microsecond timescales. It is described in detail in [111, 118]. A schematic representation of the control systems is in Fig. 3.9. We use the Trapped Ion Control Software (TrICS) developed by the Blatt group in Innsbruck as a user interface for controlling these systems and collecting experimental data.

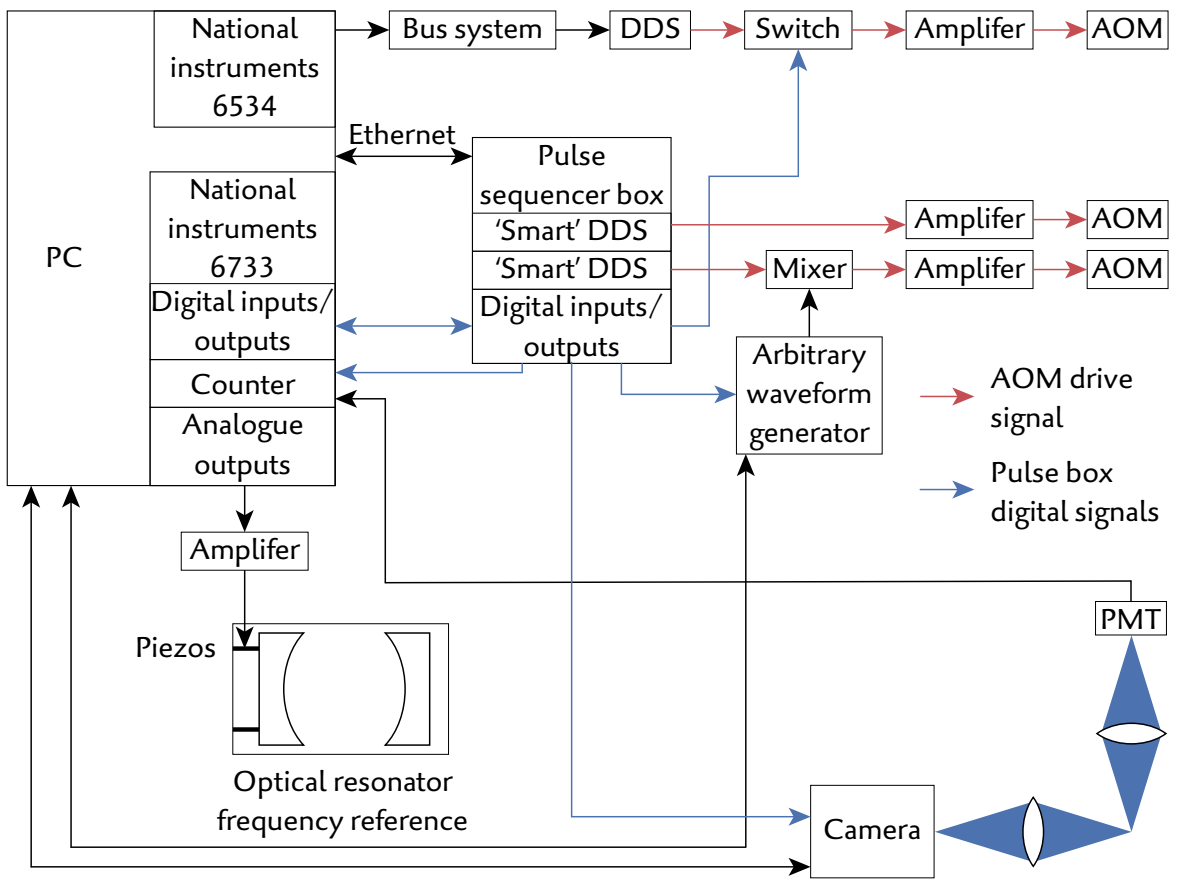

Figure 3.9: Schematic of electronic control systems.

During a single experiment run a sequence of laser pulses is applied to trapped ions and then the ions' states are measured (Section 3.1). The laser pulses are produced by driving AOMs with RF 
signals (Section 3.3.2). RF signals with $10 \mathrm{~ns}$ timing resolution are output directly from the pulse sequencer box or they are produced by switching the RF signals from the bus system using digital signals from the pulse sequencer box. Measurements are choreographed by using digital signals from the pulse sequencer box to gate the photon counter and to trigger the EMCCD camera.

The radiofrequency AOM drive signals are produced by direct digital synthesizer (DDS) chips. The DDS chips are either programmed via the bus system or they are included in the pulse sequencer box. The "smart" DDS boards within the pulse sequencer box produce versatile AOM drive signals which allow phase-coherent frequency switching and amplitude shaping of laser pulses during a single experiment run. Phase-coherent frequency switching is required for coherent manipulation of an ion state involving different transitions [117]. The amplitude shaping has bandwidth $\approx 2 \pi \times 5 \mathrm{MHz}$. The "smart" DDSs are usually reserved for control of the $674 \mathrm{~nm}$ laser beams and the Rydberg-excitation lasers.

Digital signals from the pulse sequencer box are used to trigger and switch other devices, such as arbitrary waveform generators used to shape laser pulses with high bandwidth $(\sim 2 \pi \times 20 \mathrm{MHz})$ for coherent Rydberg excitations (Chapter 7).

Experiment runs are typically repeated $\sim 100$ times with the same experimental parameters, and sets of experiment runs are repeated as a parameter is scanned. Laser frequencies, powers and phases are scanned by reprogramming the bus system DDSs or the pulse sequencer box settings between sets of experiment runs.

The PC communicates with the bus system via National Instruments card 6534 and it communicates with the pulse sequencer box via National Instruments card 6733 and an Ethernet connection. Card 6733 includes the counter which records signals from the PMT (see Section 3.1.4) and it supplies analogue voltages which control the lengths of the optical resonators used as frequency references (Section 3.3.4). 

This chapter is concerned with the first Rydberg ion experiments carried out in our laboratory. We use a two-photon excitation scheme, which differs notably from the single-photon excitation scheme used in the experiment in Mainz, described in Section 1.3.2. The twophoton excitation scheme allows for a lower light-induced coupling between internal and external degrees of freedom compared with the single-photon excitation scheme (Section 4.3). This allows us to observe narrower Rydberg resonances than the Mainz experiment and to resolve resonance structure.

The experimental techniques developed in this chapter allow us to investigate Rydberg ion-trap effects (Chapter 6) and to coherently control Rydberg ions (Chapter 7).

\section{I THE FIRST EXCITATION STEP}

In our experiment a trapped ${ }^{88} \mathrm{Sr}^{+}$ion is excited to Rydberg S- and Dstates by two-photon excitation, driven by $243 \mathrm{~nm}$ and $\sim 307 \mathrm{~nm}$ laser light (see Fig. 3.1). $243 \mathrm{~nm}$ laser light drives the first excitation step from the metastable state $4 \mathrm{D}_{5 / 2}$ near to $6 \mathrm{P}_{3 / 2}$ or from the metastable state $4 \mathrm{D}_{3 / 2}$ near to $6 \mathrm{P}_{1 / 2}$.

\subsection{1 $4 \mathrm{D}_{5 / 2} \leftrightarrow 6 \mathrm{P}_{3 / 2}$ transition}

The $4 \mathrm{D}_{5 / 2} \leftrightarrow 6 \mathrm{P}_{3 / 2}$ transition is probed as follows [schematic in Fig. 4.1(a)]:

o. The ion is prepared in a Zeeman sublevel of $4 \mathrm{D}_{5 / 2}$, as described in Section 3.1.3.

1. The $4 \mathrm{D}_{5 / 2} \leftrightarrow 6 \mathrm{P}_{3 / 2}$ transition is driven by $243 \mathrm{~nm}$ laser light; excited population decays mostly to $5 S_{1 / 2}$. 
2. Electron shelving: Detection of fluorescence from the $5 S_{1 / 2} \leftrightarrow$ $5 \mathrm{P}_{1 / 2}$ transition heralds successful excitation to $6 \mathrm{P}_{3 / 2}$ (see Section 3.1.4).

Using calculated electronic wavefunctions (Section 2.2) we find population in $6 \mathrm{P}_{3 / 2}$ decays mostly to $5 S_{1 / 2}$ by multi-step decay processes. The resonance lineshape is shown in Fig. 4.I(b).

a

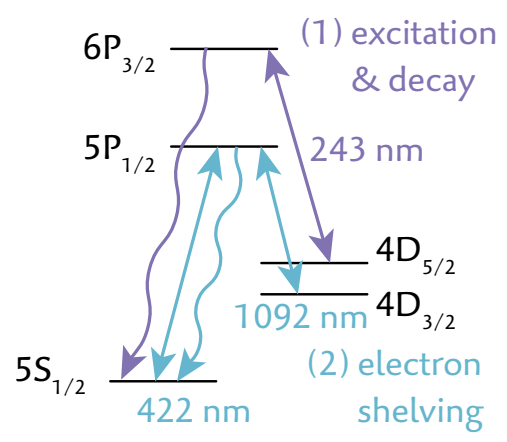

b

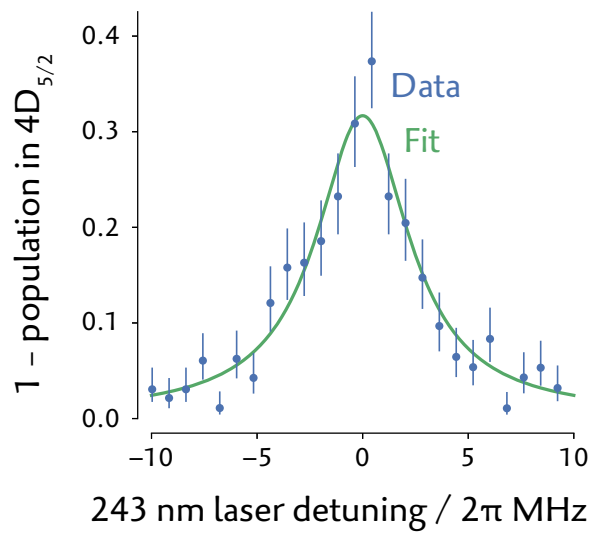

Figure 4.1: Measurement of the $4 \mathrm{D}_{5 / 2} \leftrightarrow 6 \mathrm{P}_{3 / 2}$ resonance. (a) First $243 \mathrm{~nm}$ light drives the transition to $6 \mathrm{P}_{3 / 2}$ and population in $6 \mathrm{P}_{3 / 2}$ decays to $5 S_{1 / 2}$, the ion state is then measured by electron shelving. (b) Because detection of successful excitation involves absorption of a $243 \mathrm{~nm}$ photon and optical pumping out of $4 \mathrm{D}_{5 / 2}$, the resonance lineshape is the Lorentzian absorption profile in the exponent of the exponential function. Error bars indicate quantum projection noise ( $68 \%$ confidence interval).

The excitation proceeds incoherently because the transition Rabi frequency is much lower than the $6 \mathrm{P}_{3 / 2}$ decay rate. The entire process can be viewed as optical pumping from $4 \mathrm{D}_{5 / 2}$ to $5 \mathrm{~S}_{1 / 2}$ and to $4 \mathrm{D}_{3 / 2}$ via $6 \mathrm{P}_{3 / 2}$, and the population in $4 \mathrm{D}_{5 / 2}$ decays according to

$$
\mathrm{P}_{4 \mathrm{D}_{5 / 2}}=\mathrm{e}^{-\mathcal{R}\left(\omega_{243}\right) t_{\mathrm{ex}}},
$$

where the frequency-dependent absorption rate

$$
\mathcal{R}\left(\omega_{243}\right)=\frac{\Omega_{1}^{2}}{\Gamma_{6 \mathrm{P}_{3 / 2}}}\left(1+\frac{4\left(\omega_{243}-\omega_{\mathrm{o}}\right)^{2}}{\Gamma_{6 \mathrm{P}_{3 / 2}}^{2}}\right)^{-1}
$$


and $t_{e x}$ is the excitation time, $\Omega_{1}$ is the transition Rabi frequency, $\Gamma_{6 P_{3 / 2}}$ is the natural decay rate of $6 \mathrm{P}_{3 / 2}, \omega_{243}$ is the $243 \mathrm{~nm}$ laser frequency and $\omega_{\mathrm{o}}$ is the resonance frequency of the transition. $\mathcal{R}\left(\omega_{243}\right)$ has a Lorentzian profile with linewidth $\Gamma_{6 \mathrm{P}_{3 / 2}}$ unless there is Doppler broadening. From the fit in Fig. 4.I (b) we extract $\Gamma_{6 \mathrm{P}_{3 / 2}}=2 \pi \times(4.9 \pm$ $0.4) \mathrm{MHz}$, the theoretically-determined values are $2 \pi \times 4.71 \mathrm{MHz}$ [82] and $2 \pi \times 4.26 \mathrm{MHz}$ [83]. We also extract $\Omega_{1}$ and $\omega_{0}$ from the fit.

\subsection{2 $4 \mathrm{D}_{3 / 2} \leftrightarrow 6 \mathrm{P}_{1 / 2}$ transition}

The $4 \mathrm{D}_{3 / 2} \rightarrow 6 \mathrm{P}_{1 / 2}$ transition is probed as follows [schematic in Fig. 4.2(a)]:

o. The ion is prepared in a mixture of the four Zeeman sublevels of $4 \mathrm{D}_{3 / 2}$ (Section 3.1.3).

1. Laser light at $243 \mathrm{~nm}$ drives the $4 \mathrm{D}_{3 / 2} \leftrightarrow 6 \mathrm{P}_{1 / 2}$ transition, population excited to $6 \mathrm{P}_{1 / 2}$ decays mostly to $5 \mathrm{~S}_{1 / 2}$.

2. Population in $5 S_{1 / 2}$ is transferred to $4 D_{5 / 2}$.

3. Electron shelving: Successful excitation to $6 \mathrm{P}_{1 / 2}$ is heralded when no fluorescence from the $5 \mathrm{~S}_{1 / 2} \leftrightarrow 5 \mathrm{P}_{1 / 2}$ transition is detected (Section 3.1.4).

Using calculated electronic wavefunctions (Section 2.2) we find population in $6 \mathrm{P}_{1 / 2}$ decays mostly to $5 \mathrm{~S}_{1 / 2}$ by multi-step decay processes. Resonance lineshapes are shown in Fig. 4.2(b).

At the position of the ion the $243 \mathrm{~nm}$ laser beam propagation is parallel with the magnetic field and thus laser light with circular polarisation drives the two $4 \mathrm{D}_{3 / 2}, \mathrm{~m}_{\mathrm{J}}=-\frac{3}{2} \rightarrow 6 \mathrm{P}_{1 / 2}, \mathrm{~m}_{\mathrm{J}}=-\frac{1}{2}$ and $4 \mathrm{D}_{3 / 2}, \mathrm{~m}_{\mathrm{J}}=-\frac{1}{2} \rightarrow 6 \mathrm{P}_{1 / 2}, \mathrm{~m}_{\mathrm{J}}=\frac{1}{2} \sigma^{+}$transitions or the two respective $\sigma^{-}$transitions.

Each peak in Fig. 4.2(b) corresponds to two overlapping resonances which are not individually resolved because the $2 \pi \times 670 \mathrm{kHz}-$ Zeeman splitting of the resonance frequencies (in a magnetic field of strength $0.36 \mathrm{mT}^{1}$ ) is much less than the theory value of the resonance

I Throughout this work Landé g-factors are used for magnetic moments. 

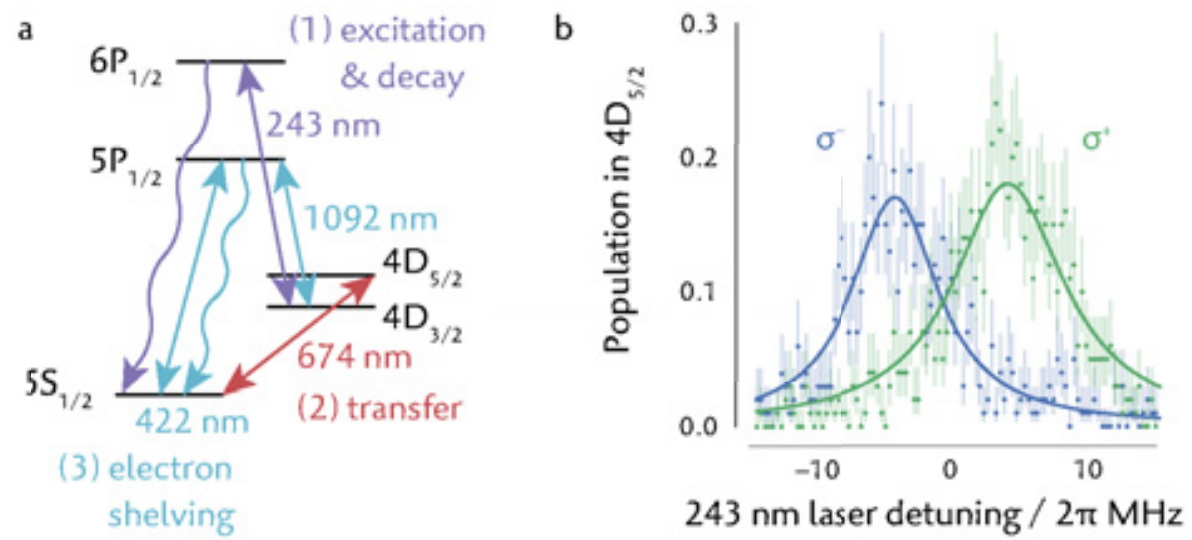

Figure 4.2: Measurement of the $4 \mathrm{D}_{3 / 2} \leftrightarrow 6 \mathrm{P}_{1 / 2}$ resonance. (a) First $243 \mathrm{~nm}$ light drives the transition to $6 \mathrm{P}_{1 / 2}$ and population in $6 \mathrm{P}_{1 / 2}$ decays to $5 S_{1 / 2}$, then $674 \mathrm{~nm}$ light transfers population from $5 S_{1 / 2}$ to $4 D_{5 / 2}$, finally the ion state is measured. (b) Either $\sigma^{+}$or $\sigma^{-}$ transitions are driven by using the appropriate laser polarisation. The transitions are non-degenerate due to the Zeeman splittings of the atomic sublevels. The solid curves are fits to the experimental data. Error bars indicate quantum projection noise $(68 \%$ confidence interval).

linewidth $\Gamma_{6 \mathrm{P}_{1 / 2}} \approx 2 \pi \times 4 \mathrm{MHz}[82,83]$. Each peak in Fig. 4.2(b) is fit using a similar method as in Section 4.1.1, only the absorption rate $\mathcal{R}\left(\omega_{243}\right)$ includes two Lorentzian functions for the two transitions weighted by their respective Clebsch-Gordan coefficients. The fit function accounts for the imperfect population transfer from $5 S_{1 / 2} \rightarrow$ $4 D_{5 / 2}$ and assumes the initial population is uniformly spread across the $4 D_{3 / 2}$ sublevels. The frequency difference of the two peaks is consistent with the expected Zeeman splitting between the pairs of transitions. The resonance linewidth inferred from the data $2 \pi \times$ $(7.2 \pm 0.4) \mathrm{MHz}$ exceeds the theory value $\approx 2 \pi \times 4 \mathrm{MHz}\left[8_{2}, 8_{3}\right]$, possibly due to Doppler broadening. 


\subsection{FOCUSSING RYDBERG EXCITATION LASERS ON AN ION}

\subsubsection{UV laser setups at the experiment chamber}

Rydberg excitation laser light is delivered near to the experiment chamber through single-mode fibres, as described in Section 3.3.3.

After each fibre each laser beam propagates through two identical achromatic lenses and into the experiment chamber. The laser beams propagate through holes in the endcap electrodes [Fig. 3.3(a)]. The lenses image the fibre modes (with diameters $\sim 8 \mu \mathrm{m}$ ) onto trapped ions with magnification 1 . Because the lenses are achromatic the laser beams may be sent from either direction, or both may be sent from the same direction. A single ion is used to optimise the focussing of each Rydberg excitation laser as described in the following subsections.

\subsubsection{Focussing $243 \mathrm{~nm}$ light on an ion}

The focussing of $243 \mathrm{~nm}$ light onto the ion is optimised by moving the two lenses (which are mounted on translation stages between the fibre output and the experiment chamber) and maximising the strength with which the 4D $\leftrightarrow$ 6P transitions in Section 4.I are driven.

The ion is also used to profile the laser beam: The strength with which the $243 \mathrm{~nm}$ laser drives the 4D $\leftrightarrow 6 \mathrm{P}$ transitions is recorded as the laser beam is shifted. The laser beam is shifted by displacing the second lens in the directions perpendicular to the laser beam propagation direction. Because the laser beam is collimated at the position of the second lens, displacement of this lens displaces the focus by the same amount at the position of the ion. A beam profile measured using the ion is shown in Fig. 4.3.

At the ion position the beam is elliptical, with Gaussian beam waists $w_{x}=4.9 \mu \mathrm{m}$ and $w_{y}=10.7 \mu \mathrm{m}$. The laser beam propagates through holes in the trap endcaps which limit the numerical aperture to 0.058 . In principle a focus with waist $1.3 \mu \mathrm{m}$ may be attained with an improved optical setup. 


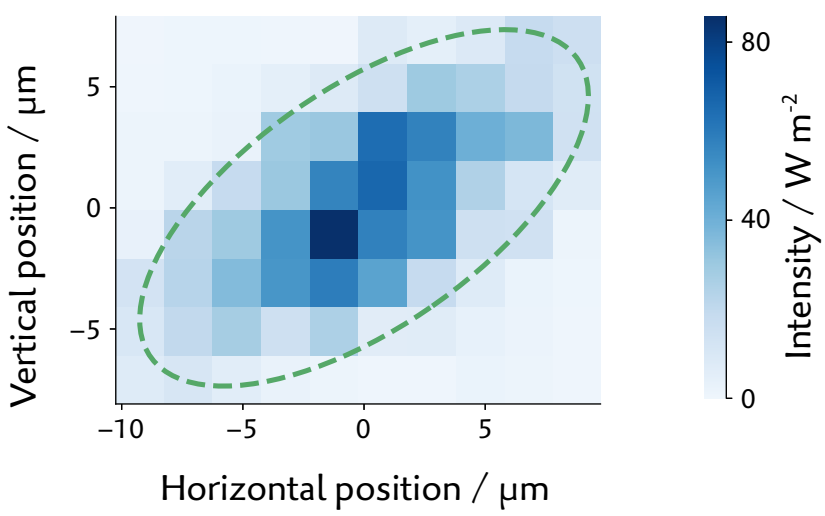

Figure 4.3: The $243 \mathrm{~nm}$ laser beam profile at the position of the ion is determined by moving the laser beam and measuring the strength with which the $4 \mathrm{D}_{5 / 2} \leftrightarrow 6 \mathrm{P}_{3 / 2}$ transition is driven. The dashed green line represents the $1 / e^{2}$ beam waist. The relative intensity is converted to the absolute laser light intensity with $44 \%$ uncertainty. This uncertainty arises because the laser power is not measured at the ion position; rather the laser power is measured before and after the chamber and the surrounding optics, and only $32 \%$ of the laser power $\left[(1-0.44)^{2}\right]$ is transmitted through the system.

\subsubsection{Focussing $\sim 307 \mathrm{~nm}$ light on an ion}

Laser light near $307 \mathrm{~nm}$ is required for the second Rydberg excitation step. This laser light shifts the energies of $5 S_{1 / 2}$ and $4 D_{5 / 2}$ Zeeman sublevels according to the ac-Stark effect, by an amount which is proportional to the $307 \mathrm{~nm}$ laser light intensity. The focussing of $\sim 307 \mathrm{~nm}$ light onto the ion is optimised by moving the two lenses and maximising the resonance frequency shift induced onto one of the $5 \mathrm{~S}_{1 / 2} \leftrightarrow 4 \mathrm{D}_{5 / 2}$ transitions. The resonance frequency shift is measured in a Ramsey experiment.

This Ramsey-type method is also used to measure the $307 \mathrm{~nm}$ laser beam profile at the position of the ion, which is shown in Fig. 4.4. The beam is near-round, with Gaussian beam waists $w_{x}=5.3 \mu \mathrm{m}$ and $w_{y}=6.1 \mu \mathrm{m}$. With the current setup the focus likely cannot be improved, given the expected fibre mode field diameter $\approx 8 \mu \mathrm{m}$. 


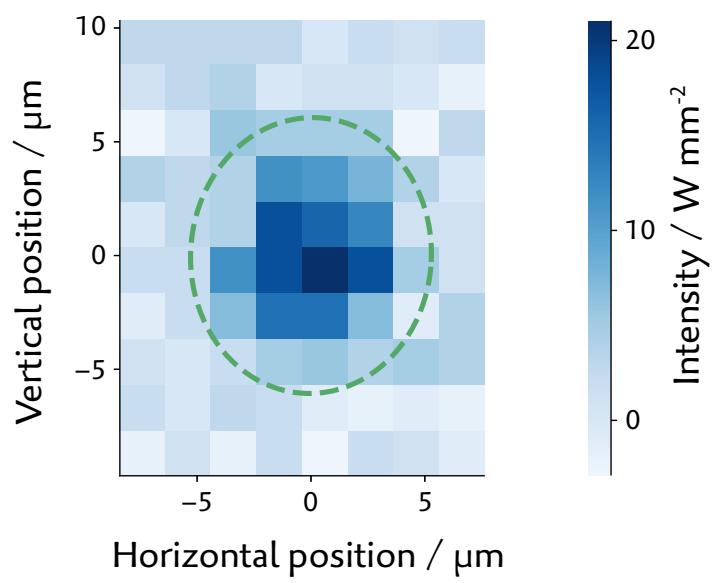

Figure 4.4: The $307 \mathrm{~nm}$ laser beam profile at the position of the ion is determined by moving the laser beam and at each position the relative $307 \mathrm{~nm}$ laser light intensity is measured in a Ramsey-type experiment. The dashed green line represents the $1 / e^{2}$ beam waist. The relative intensity is converted to the absolute laser light intensity with $16 \%$ uncertainty. This uncertainty arises because the laser power is not measured at the ion position; rather the laser power is measured before and after the chamber and the surrounding optics, and $70 \%$ of the laser power $\left[(1-0.16)^{2}\right]$ is transmitted through the system. The intensity scale includes nonphysical negative values, this is due to statistical fluctuations of the Ramsey measurement results.

This laser beam also propagates through holes in the trap endcaps which limit the numerical aperture to 0.058 , and thus a beam waist of $1.7 \mu \mathrm{m}$ can be attained for this laser beam with an improved optical setup.

\section{4 -3 TWO-PHOTON RESONANCE CONDITION}

Rydberg states are excited and detected using a similar methodology as in Section 4.1; UV laser light at $243 \mathrm{~nm}$ and $\sim 307 \mathrm{~nm}$ drives a twophoton transitions from a metastable 4D-state to a Rydberg S- or Dstate. Each UV laser is detuned from resonance to the intermediate $6 \mathrm{P}$ state by typically $2 \pi \times 200 \mathrm{MHz}$, the detunings are opposite such 
that two-photon resonance is achieved. Using calculated electronic wavefunctions (Section 2.2) we find population in Rydberg S- and $D$-states decays mostly to $5 S_{1 / 2}$ by multi-step decay processes.

The effective Rabi frequency of the two-photon transition is

$$
\Omega_{\text {eff }}=\frac{\Omega_{1} \Omega_{2}}{2 \Delta},
$$

where $\Delta$ is the magnitude of the detuning of each laser from the intermediate state. This formula is derived in Section 7.3.1.

We use $\Delta \gg \Omega_{1}, \Gamma_{6 \mathrm{P}}$ to minimise scattering off the intermediate state which causes a background signal. The rate at which population from the initial state scatters off the intermediate state is

$$
\Gamma_{\text {scat }}=\frac{\Omega_{1}^{2} \Gamma_{6 \mathrm{P}}}{4 \Delta^{2}} \text {. }
$$

Results are shown in Fig. 4.5 for the excitation from $4 \mathrm{D}_{3 / 2} \rightarrow$ $25 \mathrm{~S}_{1 / 2}$ which exemplify the two-photon resonance condition. In each dataset the frequency of the $243 \mathrm{~nm}$ laser has a different intermediatestate detuning while the frequency of the $309 \mathrm{~nm}$ laser is scanned; the Rydberg resonance structure is observed when the two-photon resonance condition is met. Each dataset shows a four-peak resonance structure corresponding to four Zeeman-split transitions, this structure is explained in Section 4.5 .

\subsection{COUNTERPROPAGATING RYDBERG-EXCITATION LASERS}

The two UV laser beams counterpropagate along the trap axis, collinear with the magnetic field [Fig. 3.3(a)]. On two-photon absorption the momentum kicks from the two photons on the ion largely cancel and the ion motion is not greatly disturbed. This means phononnumber-changing transitions (Section 3.1.1) are less likely to occur with counterpropagating laser beams than with copropagating laser beams or with a single Rydberg excitation step. More thoroughly, it is easier to achieve excitation within the Lamb-Dicke regime when counterpropagating laser beams are used. By adapting Eq. (3.1) we find the effective Lamb-Dicke parameter

$$
\eta_{\text {eff }}=\left(k_{1}-k_{2}\right) \sqrt{\frac{\hbar}{2 M \omega_{z}}},
$$




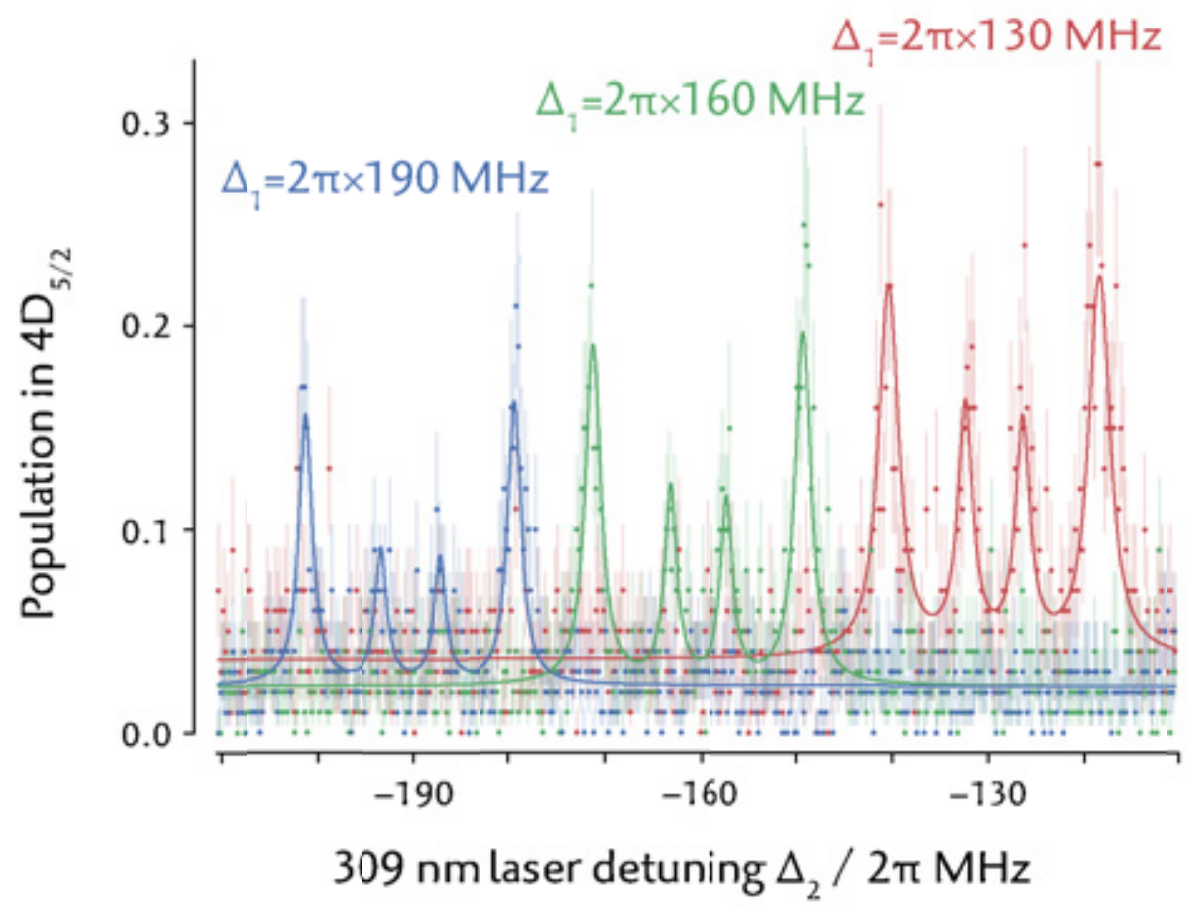

Figure 4.5: Two-photon excitation from $4 \mathrm{D}_{3 / 2}$ to $25 \mathrm{~S}_{1 / 2}$. The resonance structure is centred on the position where the two-photon detuning $\Delta_{1}+\Delta_{2}=0$. The solid lines show fits to the data. The absolute value of the detuning of the $309 \mathrm{~nm}$ laser from the $6 \mathrm{P}_{1 / 2} \leftrightarrow 25 \mathrm{~S}_{1 / 2}$ resonance is inferred from all three of the data sets. Error bars indicate quantum projection noise $(68 \%$ confidence interval).

where $k_{1}, k_{2}$ are the wavevectors of the $243 \mathrm{~nm}$ and $\sim 307 \mathrm{~nm}$ laser light, $M$ is the ${ }^{88} \mathrm{Sr}^{+}$mass, and $\omega_{z}$ is the axial trapping frequency. $\eta_{\text {eff }} \approx 0.045$ when typical axial trapping frequencies are used. After Doppler cooling the inequality in Eq. (3.2) is satisfied and the ion is in the Lamb-Dicke regime with regards the two-photon transition using counter-propagating lasers. Working within the Lamb-Dicke regime allows us to investigate Rydberg ion-trap effects (Chapter 6).

If instead the UV laser beams were copropagating, the Lamb-Dicke parameter would be 9 times larger and sub-Doppler cooling would be required to prepare an ion in the Lamb-Dicke regime. 
The opportunity to lower the effective Lamb-Dicke parameter by using counterpropagating laser beams is a major advantage of the two-photon excitation scheme. In the Mainz experiment $\sim 122 \mathrm{~nm}$ laser light drives the single-photon Rydberg-excitation transition and sub-Doppler cooling is required to prepare an ion in the Lamb-Dicke regime. Phonon-number-changing transitions are driven during Rydberg excitation in their experiment; this contributes to their relatively broad resonances (see Section 1.3.2).

\subsection{ZEEMAN-SPLIT RYDBERG S-STATE RESONANCES}

Transitions driven by the trapping electric fields (Section 6.2) are negligible for Rydberg S-states up to at least $n=50$ [49], and thus $m_{J}$ is a 'good' quantum number both for low-lying states and for the Rydberg S-states investigated in this work. The Zeeman effect describes the ion in a magnetic field; field strengths $\approx 0.3 \mathrm{mT}$ are used. ${ }^{2}$

The resonance structure obtained when a Rydberg S-state is excited is understood in terms of the Zeeman effect: The four resonance peaks in Figs. 4.5 and 4.6(a) correspond to the four transitions between sublevels of $4 \mathrm{D}_{3 / 2}$ and sublevels of $n S_{1 / 2}$. The transitions are non-degenerate because the sublevels of $4 \mathrm{D}_{3 / 2}$ and of $n S_{1 / 2}$ are Zeeman-split.

The configuration of the laser beams determines the transitions that may be driven [displayed in Fig. 4.6(b)]: The two Rydberg-excitation laser beams counterpropagate along the trap axis, collinear with the magnetic field. Each laser beam may drive $\sigma^{+}$transitions, $\sigma^{-}$transitions, or both transitions depending on the laser light polarisation. The different amplitudes of the resonance peaks result from the different Clebsch-Gordan coefficients of the constituent transitions, which are shown in Fig. 4.6(b).

A single transition between Zeeman sublevels may be driven by using the appropriate UV laser polarisations, as shown in Fig. 4.6(a), or by relying on the transition being frequency-resolved. When using high Rabi frequencies and short pulse lengths for coherent Rydberg

2 The Zeeman effect is much smaller than the fine-structure splitting until $n \sim 200$. The paramagnetic Zeeman term dominates the diamagnetic term also until $n \sim 200$. 


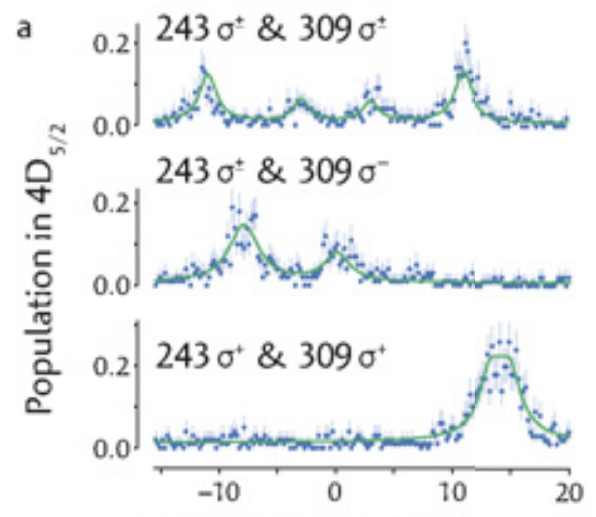

Two-photon detuning / $2 \pi \mathrm{MHz}$

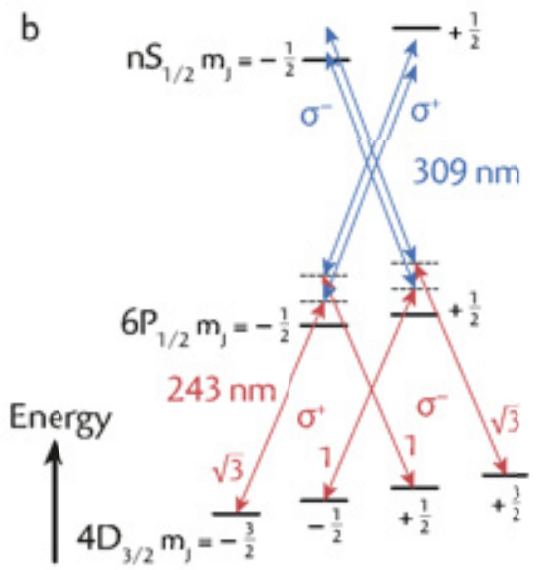

Figure 4.6: The $4 \mathrm{D}_{3 / 2} \leftrightarrow 25 \mathrm{~S}_{1 / 2}$ resonance structure results from Zeeman splitting of the $4 \mathrm{D}_{3 / 2}$ and $25 \mathrm{~S}_{1 / 2}$ sublevels. At most four non-degenerate two-photon transitions are driven, since the Rydberg excitation laser beams are collinear with the magnetic field. (a) Individual transitions are selected by using appropriate laser polarisations. The solid lines are fits to the data, the magnetic field strength is constrained to $0.356 \mathrm{mT}$ - it is independently determined from the $5 \mathrm{~S}_{1 / 2} \leftrightarrow 4 \mathrm{D}_{5 / 2}$ spectrum. The amplitudes of the peaks depend on the Clebsch-Gordan coefficients of the first transition step; the relative magnitudes of the Clebsch-Gordan coefficients are shown in (b). As the $309 \mathrm{~nm}$ laser polarisation is changed the resonances shift by $\approx 2 \pi \times 2 \mathrm{MHz}$ likely because of the ac-Stark effect. The fit to the lowest spectrum appears clipped at 0.22 due to saturation of the absorption signal; the maximum signal is limited by $\approx 25 \%$ of the population being initialised in each $4 \mathrm{D}_{3 / 2}$ sublevel and the $\approx 87 \% 5 \mathrm{~S}_{1 / 2} \rightarrow 4 \mathrm{D}_{5 / 2}$ transfer efficiency during the measurement step. Error bars indicate quantum projection noise ( $68 \%$ confidence interval).

excitation (as in Chapter 7) Fourier broadening causes the frequencyresolving power to be reduced and it is then best to select a single transition using laser polarisations. 


\subsection{RYDBERG ENERGY SERIES}

We have excited a trapped ${ }^{88} \mathrm{Sr}^{+}$ion to Rydberg S-states with principal quantum numbers in the range 25 to 57 . The state energies are determined by employing a wavelength meter $^{3}$ to measure the frequencies of the fundamental lasers used to generate the Rydberg excitation lasers. Uncertainties in the wavelength measurements limit the determination of the state energies to $\hbar 2 \pi \times 300 \mathrm{MHz}$.

The state energies $E_{n L J}$ are fit following the methodology of [67] with the Rydberg energy series formula

$$
E_{n L J}=I^{++}-\frac{Z^{2} R_{M}}{\left(n-\mu\left(E_{n L J}\right)\right)^{2}},
$$

where $\mathrm{I}^{++}$is the second ionisation threshold and the Rydberg constant for ${ }^{88} \mathrm{Sr}^{+}$with mass $\mathrm{M}=87.9 \mathrm{u}$ [119] is

$$
R_{M}=R_{\infty} \frac{M}{M+m_{e}},
$$

$R_{\infty}$ is the Rydberg constant, $m_{e}$ is the electron mass, and the quantum defect is a linear function of the binding energy

$$
\begin{aligned}
\mu\left(E_{n L J}\right) & =\mu\left(I^{++}\right)+\frac{\partial \mu}{\partial E}\left(E_{n L J}-I^{++}\right) \\
& =\mu\left(I^{++}\right)-\frac{\partial \mu}{\partial E} \frac{Z^{2} R_{M}}{\left(n-\mu\left(E_{n L J}\right)\right)^{2}} \\
& \approx \mu\left(I^{++}\right)-\frac{\partial \mu}{\partial E} \frac{Z^{2} R_{M}}{\left(n-\mu\left(I^{++}\right)\right)^{2}} .
\end{aligned}
$$

The energy series and the mismatch between measured values and the fit are shown in Fig. 4.7. Resonance frequency shifts due to trap effects (Chapter 6) and the ac-Stark effect are small compared with the wavelength meter uncertainty.

The energy series of Rydberg S-, D-, F- and G-states of $\mathrm{Sr}^{+}$in free space were previously measured by Lange et al. [67]. They measured states energies accurate to $\hbar 2 \pi \times 6 \mathrm{GHz}$, limited by the linewidth of

3 HighFinesse WS6-200 

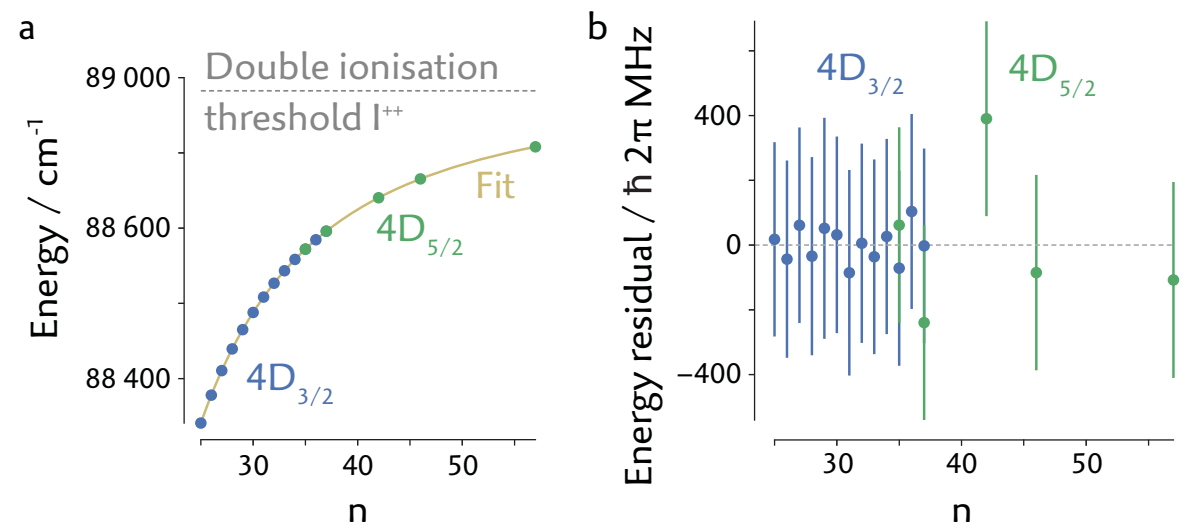

Figure 4.7: ${ }^{88} \mathrm{Sr}^{+}$S-state energies are described well by the Rydberg energy series formula. State energies are measured using excitation from $4 D_{3 / 2}$ and from $4 D_{5 / 2}$. (a) Binding energies scale as $n^{*-2}$. (b) Differences between the data and the fit are generally within the $68 \%$ confidence interval indicated by the error bars. Experimental uncertainties are likely correlated; they are dominated by the wavelength meter which contributes $2 \pi \times 300 \mathrm{MHz}$ to the error bars.

their Rydberg-excitation laser at $\sim 280 \mathrm{~nm}$. Our estimates of the double ionisation threshold and the S-state quantum defect are consistent with the previous experimental investigation, as shown in Table 4.1.

We have also excited two Rydberg D-states and estimate quantum defects $\mu\left(24 \mathrm{D}_{3 / 2}\right)=1.4563 \pm 0.0003$ and $\mu\left(27 \mathrm{D}_{3 / 2}\right)=1.4563 \pm$ 0.0004 , which are consistent with the values from Lange et al.

Improved spectroscopy allows for more accurate calculations of electronic wavefunctions (Section 2.2) and thus more accurate theoretical predictions of Rydberg state properties. Our institute has recently purchased a wavelength meter accurate to $2 \pi \times 2 \mathrm{MHz} .{ }^{4}$ We plan to repeat the energy series measurement of Rydberg $S$-states, as well as Rydberg P- and D-states. ${ }^{5}$

4 HighFinesse WS8-2

5 Rydberg P-states were recently excited in our experiment using two UV photons and one MW photon; this work forms part of the PhD thesis project of Fabian Pokorny, see the Outlook in Chapter 8. 


\begin{tabular}{lcc}
\hline & This work & Lange et al. [67] \\
\hline $\begin{array}{l}\text { Double ionisation threshold } \\
\mathrm{I}^{++} / \mathrm{cm}^{-1}\end{array}$ & $88965.022 \pm 0.011$ & $88965.18 \pm 0.02$ \\
$\begin{array}{l}\mathrm{S} \text {-series quantum defect } \\
\mu\left(\mathrm{I}^{++}\right)\end{array}$ & $2.7063 \pm 0.0009$ & $2.707 \pm 0.002$ \\
$\begin{array}{l}\text { S-series quantum defect } \\
\text { gradient }(\partial \mu / \partial \mathrm{E}) / \mathrm{Ryd}^{-1}\end{array}$ & $-0.04 \pm 0.09$ & $-0.055 \pm 0.015$ \\
\hline
\end{tabular}

Table 4.1: The ${ }^{88} \mathrm{Sr}^{+}$double ionisation threshold and S-state quantum defect determined in this work is consistent with previous work [67]. Our value of $\mathrm{I}^{++}$uses the $4 \mathrm{D}_{5 / 2}$ level energy from [99]. 
In our experiment a trapped ${ }^{88} \mathrm{Sr}^{+}$ion is lost by double ionisation after typically several hundred excitations to a Rydberg state. Similarly double ionisation occurs in $\sim 0.3 \%$ excitations to a Rydberg state in the Mainz experiment [75]. Double ionisation thus presents an obstacle in trapped Rydberg ion experiments, discussed in Section 5.1. We know the ion is lost by double ionisation by measuring the final product to be ${ }^{88} \mathrm{Sr}^{2+}$, these measurements are described in Section 5.2.

We suspect Rydberg states with higher principal quantum numbers are more prone to double ionisation loss and that blackbody radiation increases the likelihood of double ionisation, though we have yet to carry out a systematic investigation of the effects of different parameters on the ion loss rate. Müller et al. [49] briefly discuss double ionisation driven by the electric fields of the trap.

\subsection{OBSTACLES PRESENTED BY ION LOSS}

Ion loss has made data collection in this experiment cumbersome. Until recently ion loading was carried out using a resistively-heated oven (Section 3.2.7) and each double ionisation event caused the experiment to be interrupted by around 20 minutes. During these recurrent interruptions the frequencies of the Rydberg excitation lasers drifted by $\sim 2 \pi \times 300 \mathrm{kHz}$ because the lengths of the optical resonator frequency references (Section 3.3.4) are not perfectly stable. Interruptions due to ion loss are now reduced to less than 30 seconds through introduction of laser ablation loading. A Rydberg ion quantum computer would certainly require faster ablation loading [120, 121] or fast loading from an ion reservoir [75].

Ion loss threatens the gate fidelity in a Rydberg ion quantum computer, though it may be tolerable. Provided the loss rate is below a threshold value, quantum error correction protocols may be used 
to correct for ion loss. For instance a single ionic qubit lost due to double ionisation may be replaced by shuttling an ion in state $|1\rangle$ from a reservoir [48], the qubit state may then be corrected using the same quantum error correction protocols which correct for qubit state decay $|0\rangle \rightarrow|1\rangle$ [122].

Atom loss presents similar difficulties in neutral Rydberg atom experiments $[52,56]$. In optical traps intense trapping fields drive photoionisation of Rydberg states at rates which may exceed radiative decay rates [123-125]. To get around this problem optical traps are routinely switched off during Rydberg excitation, ${ }^{1}$ and some researchers are pursuing blue-detuned (dark) optical traps [56, 80]. Blackbody radiation also drives photoionisation of Rydberg atoms [126]. Additionally ground-state atoms are lost from optical traps due to collisions with background gas particles.

\subsection{MEASUREMENTS OF THE LOSS PRODUCT}

We know the ion is lost by double ionisation because we measure the loss product to be ${ }^{88} \mathrm{Sr}^{2+}$. In Section 5.2.1 we establish the loss product has charge $+2 \mathrm{e}$ by imaging ions on an electron-multiplying charge-coupled device (EMCCD) camera. In Section 5.2.2 we find the loss product has the same mass as ${ }^{88} \mathrm{Sr}^{+}$by measuring motional mode frequencies.

We also find that ions may be doubly-ionised not only from a Rydberg state, but also from a metastable 4D state when intense $243 \mathrm{~nm}$ laser light is used. In such cases double ionisation likely proceeds via absorption of two $243 \mathrm{~nm}$ photons.

\subsubsection{Imaging ions with an EMCCD camera}

${ }^{88} \mathrm{Sr}^{+}$ions scatter $422 \mathrm{~nm}$ laser light, and are imaged on an EMCCD camera, as shown in Fig. 5.1. Multiple trapped ions crystallise as a linear string along the trap axis when the confining potential is

1 Switching off the trap is not an option in our experiment because Coulomb repulsion between two ions initially separated by $5 \mu \mathrm{m}$ would cause them to become separated by $30 \mu \mathrm{m}$ in just $1 \mu \mathrm{s}$. 


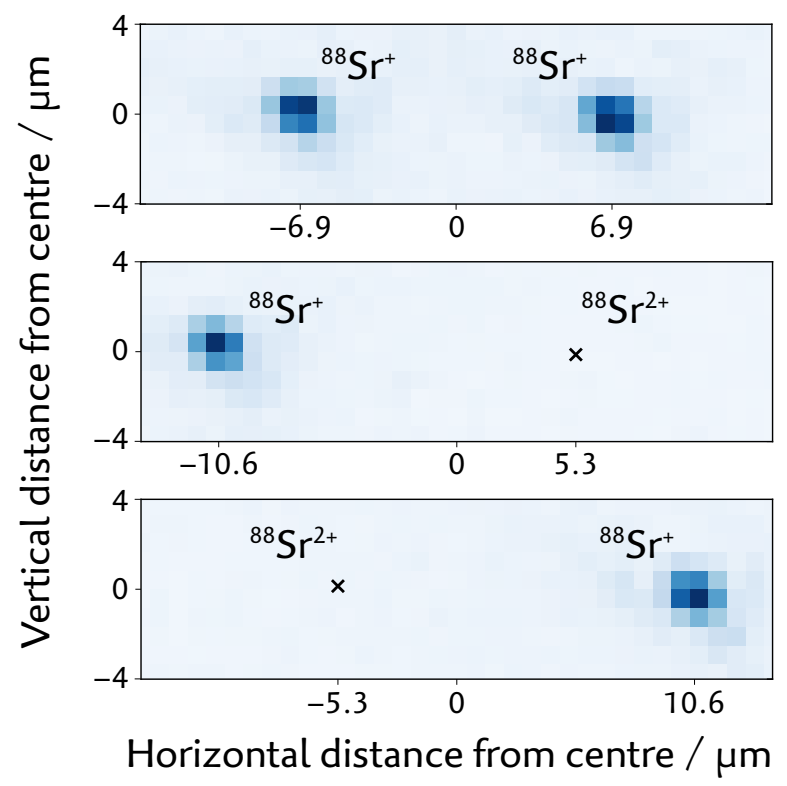

Figure 5.1: ${ }^{88} \mathrm{Sr}^{+} \_{ }^{88} \mathrm{Sr}^{+}$ion crystal compared with ${ }^{88} \mathrm{Sr}^{+}-{ }^{88} \mathrm{Sr}^{2+}$ crystals. ${ }^{88} \mathrm{Sr}^{+}$ions are imaged on an EMCCD camera using scattered $422 \mathrm{~nm}$ laser light, ${ }^{88} \mathrm{Sr}^{2+}$ ions are not observed directly. In the lower two images the distance of the ${ }^{88} \mathrm{Sr}^{+}$ion from the trap centre is consistent with a mixed crystal containing a doublycharged ion. In each image the $X$ marks the spot where the ${ }^{88} \mathrm{Sr}^{2+}$ ion lies.

much weaker in the axial direction than the radial directions. The equilibrium positions of multiple trapped ions are found by balancing the Coulomb repulsion between the ions and the confining harmonic trap forces [127].

Two ions each with charge $+\mathrm{e}$ have equilibrium positions $\pm 2^{-2 / 3} \mathrm{~L}$ relative to the trap centre, where

$$
L=\sqrt[3]{\frac{e^{2}}{4 \pi \epsilon_{0} M \omega_{z}^{2}}},
$$

$e$ is the elementary charge, $M$ is the ion mass and $\omega_{z}$ is the axial mode frequency. $\omega_{z}$ is determined by spectroscopy on the qubit transition or by 'tickling' the trap electrons (see Section 5.2.2). By imaging two 
ions on the EMCCD camera $^{2}$ the image magnification is determined to be 19.2 .

The equilibrium positions of ions with different charges is determined by generalising the calculation in [127]. An ion with charge $+e$ and an ion with charge $+2 e$ form crystals with equilibrium positions $\left\{ \pm 2 \times 3^{-2 / 3} \mathrm{~L}, \mp 3^{-2 / 3} \mathrm{~L}\right\}$ relative to the trap centre.

We start with two trapped ${ }^{8} \mathrm{Sr}^{+}$ions, shown in Fig. 5.1. We then alter one of the ions during Rydberg excitation. The altered ion remains trapped, although it does not scatter $422 \mathrm{~nm}$ laser light. The unmodified ${ }^{88} \mathrm{Sr}^{+}$ion is still visible on the EMCCD camera and may be stably trapped at positions $\pm 2 \times 3^{-2 / 3} \mathrm{~L}$, as shown in the lower images of Fig. 5.1. From this we determine the altered ion (which is not imaged) has +2 e charge.

Crystals consisting of one ion with charge $+e$ and one ion with charge $+2 e$ are also obtained when we begin with only one ${ }^{88} \mathrm{Sr}^{+}$ion in the trap and then alter it during Rydberg excitation before loading another ${ }^{88} \mathrm{Sr}^{+}$ion into the trap. This indicates the double ionisation process does not rely upon having two ions in the trap. The same crystal is also obtained when intense $243 \mathrm{~nm}$ laser light is used to doubly-ionise an ion and no $307 \mathrm{~nm}$ laser light is employed.

The equilibrium ion positions are obtained by balancing electrostatic forces; they do not depend upon ion masses. Eq. (5.1) shows no mass dependence, since $\omega^{2} \sim \frac{1}{M}$. The mass of the doubly-charged ion is determined by measuring motional mode frequencies, as is described in the following section.

\subsubsection{Measuring the axial mode frequency by tickling electrodes}

We measure the mass of the altered ion to determine the loss product is ${ }^{88} \mathrm{Sr}^{2+}$ rather than some other (possibly molecular) doubly-charged species. We measure the mass of the altered ion with accuracy better than $1 \mathrm{u}$, and find the mass is consistent with the mass of ${ }^{88} \mathrm{Sr}^{+}$. We do so by measuring the frequency of a motional mode shared by a ${ }^{88} \mathrm{Sr}^{+}$ion and the doubly-charged loss product.

2 Andor iXon3 897 with pixel size $16 \mu \mathrm{m}$. 
The motional modes of $\mathrm{N}$ ions of the same mass and charge confined in a harmonic potential and arranged in a linear string is found in [127] by first using the potential of the N-ion system to find the equilibrium positions $\left\{z_{i, 0}\right\}$, then writing the Lagrangian in terms of small displacements from the equilibrium positions; for the $i^{\text {th }}$ ion the displacement $\mathrm{q}_{i}(\mathrm{t})=z_{\mathfrak{i}}(\mathrm{t})-z_{i, 0}$. Generalising this to a string of ions with different masses and different charges involves writing the Lagrangian in terms of mass-weighted displacements $p_{i}(t)=\sqrt{M_{i}} q_{i}(t)$. The same rescaling is applied in [128] to find the motional modes of different species of ions in an anharmonic trap.

Two ions with the same mass and the same charge trapped in a harmonic trap with frequency $\omega$ have motional modes with frequencies $\omega$ (the centre-of-mass mode) and $\sqrt{3} \omega$ (the breathing mode). If the mass and charge of one of the ions is different, the motional mode frequencies change. We calculate the centre-of-mass mode frequencies for one ${ }^{88} \mathrm{Sr}^{+}$ion (charge $+\mathrm{e}$, mass $87.9 \mathrm{u}$ [119]) trapped together with an ion with charge $+2 e$ and mass $M_{2}$; results are shown in Fig. 5.2. A

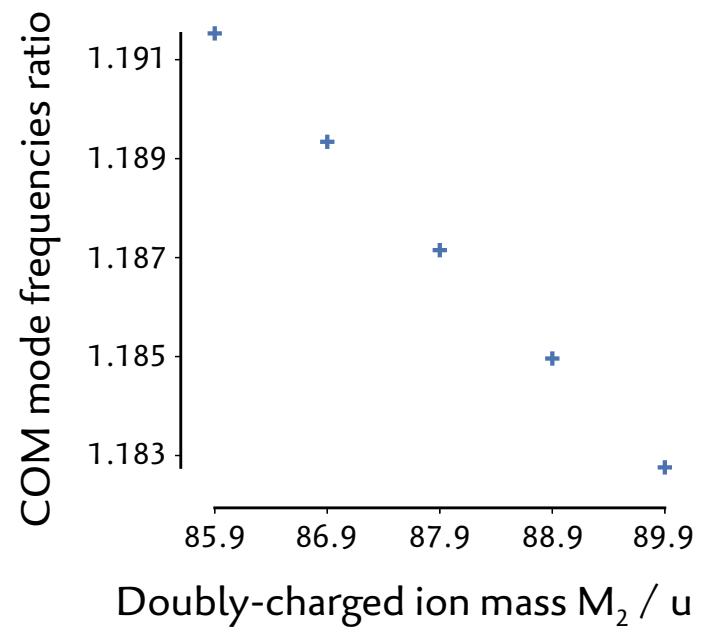

Figure 5.2: Motional mode frequencies depend on the ion masses. Shown here is the calculated ratio of the centre-of-mass mode frequency for a ${ }^{88} \mathrm{Sr}^{+}{ }^{88} \mathrm{Sr}^{+}$crystal and a mixed crystal containing ${ }^{88} \mathrm{Sr}^{+}$ and a doubly-charged ion of variable mass $M_{2}$. This ratio is measured in the experiment to find that the mass of the loss product is consistent with the mass of ${ }^{88} \mathrm{Sr}^{+}(87.9 \mathrm{u}$ [119]). 
change in $M_{2}$ of $1 \mathrm{u}$ results in a change in the mixed-species centre-ofmass mode frequency of around $2 \times 10^{-3} \times \omega$. In the experiment we measure the centre-of-mass frequencies of both the ${ }^{88} \mathrm{Sr}^{+}{ }^{88} \mathrm{Sr}^{+}$crystal and the mixed-species crystal. We determine the ratio between these frequencies with accuracy $<2 \times 10^{-4}$ and find no significant difference between the loss product mass $M_{2}$ and the ${ }^{88} \mathrm{Sr}^{+}$mass $87.9 \mathrm{u}$. In this way we determine the loss product is ${ }^{88} \mathrm{Sr}^{2+}$.

The motional frequencies can be determined from the frequencies of motional sidebands in the $5 S_{1 / 2} \leftrightarrow 4 D_{5 / 2}$ spectrum, however this requires ac-Stark shifts to be carefully accounted for. Instead we use the 'tickle' method [129]: a RF voltage is applied to the endcap electrodes, if it is resonant with a motional mode in the axial direction the axial motion is driven and the width of the ions in the EMCCD camera image is increased. The widths of the ions are recorded as the frequency of the 'tickle' voltage is scanned and in this fashion the motional mode frequencies are accurately measured. Typical results are shown in Fig. 5.3.

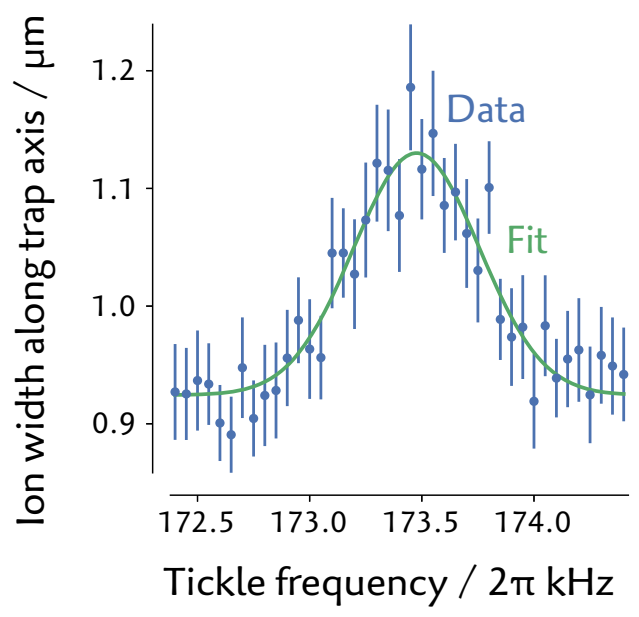

Figure 5.3: A driving field causes the ion crystal to heat if the field is resonant with a motional mode. The ions then become diffuse in the image. The data displayed is generated by fitting image data by a two-dimensional Gaussian function; error bars indicate fit uncertainties (68\% confidence interval). This data is in turn fit by the Gaussian function shown in green; motional mode frequencies are then determined to 1 part in 7000. 
Rydberg states are extremely sensitive to electric fields. In the seminal theoretical investigation by Müller et al. [49] effects of strong electric trapping fields on highly-sensitive Rydberg ions were predicted. Experimental observation of these effects is presented here. In this chapter we also investigate a Rydberg ion in trapping regimes beyond the consideration of Müller et al.; both theoretically and experimentally.

Two classes of trap effects emerge, one related to the Rydberg ion electric polarisability, the other related to the Rydberg ion electric quadrupole moment.

The Rydberg state polarisability scales as $n^{7}$, this makes Rydberg atoms sensitive electric field probes [14]. Effects of the trapping electric fields on highly-polarisable Rydberg ions are explored in Section 6.1.

Rydberg states with $\mathrm{J}>\frac{1}{2}$ have large electric quadrupole moments, which scale as $n^{4}$. Effects of the trapping electric quadrupole fields on states with large electric quadrupole moments (in particular a Rydberg $D_{3 / 2}$ state) are explored in Section 6.2. This second class of effects is negligible for $\mathrm{J}=\frac{1}{2}$ states, namely $n S_{1 / 2}$ and $n P_{1 / 2}$, with $n<50$ [49].

\subsection{EFFECTS ON HIGHLY-POLARISABLE RYDBERG IONS}

Owing to their large polarisabilities, Rydberg ions experience a different trapping pseudopotential than ions in low-lying states. This leads to different effects, which depend on whether or not the nulls of the oscillating and static electric quadrupole fields overlap.

Trap effects were theoretically studied in [49] for a trap in which the electric field nulls overlap. This corresponds to the case with 
no excess micromotion. We experimentally investigated these effects [130], the results are presented in Section 6.1.1.

We have also experimentally investigated the effects which appear when the field nulls do not overlap, and explain the results by extending the theory. When the field nulls do not overlap there is excess micromotion in the system. This work is described in Section 6.1.2.

\subsubsection{With overlapping quadrupole field nulls}

Due to the large Rydberg state electric polarisability, the radial trapping frequencies of a Rydberg ion are different to the radial trapping frequencies of an ion in a low-lying state. This means the energy required for Rydberg excitation depends on the number of phonons in radial motional modes. Before the experimental results are presented, this effect is described using a semi-classical approach. A full quantum mechanical description is found in $[49,68,130]$.

\section{Description using semi-classical theory}

Near the centre of a linear Paul trap the electric trapping potential $\Phi$ is composed of two electric quadrupole potentials, one which oscillates and one which is static:

$$
\Phi=\alpha \cos \Omega_{\mathrm{rf}} \mathrm{t}\left(\mathrm{x}^{2}-\mathrm{y}^{2}\right)-\beta\left[(1+\epsilon) x^{2}+(1-\epsilon) \mathrm{y}^{2}-2 z^{2}\right],
$$

where $\alpha$ is the time-dependent field gradient which oscillates with radiofrequency $\Omega_{\mathrm{rf}}, \beta$ is the static field gradient, and the parameter $\epsilon$ breaks the axial symmetry of the trap and lifts the radial mode degeneracy.

In the axial direction the trapping potential is harmonic with frequency $\omega_{z}$ given by

$$
\omega_{z}^{2}=\frac{4 e \beta}{M},
$$

where $e$ is the elementary charge (i.e. the ion charge) and $M$ is the ion mass.

The radial motion of an ion near the centre of the trapping potential is described in terms of the Mathieu equation. When $\beta \ll \alpha \ll \frac{M \Omega_{\mathrm{rf}}^{2}}{4 e}$ 
the first-order solution describes ion motion in each radial direction composed of two parts: harmonic motion with frequency $\omega_{x}, \omega_{y}$ called secular motion and driven motion with frequency $\Omega_{\mathrm{rf}}$ called micromotion, where

$$
\begin{aligned}
& \omega_{x}^{2}=2\left(\frac{e \alpha}{M \Omega_{\mathrm{rf}}}\right)^{2}-\frac{2 e \beta(1+\epsilon)}{M}, \\
& \omega_{y}^{2}=2\left(\frac{e \alpha}{M \Omega_{\mathrm{rf}}}\right)^{2}-\frac{2 e \beta(1-\epsilon)}{M} .
\end{aligned}
$$

The secular approximation involves neglecting the fast oscillating micromotion and interpreting the secular motion as generated by a time-independent harmonic pseudopotential

$$
\mathrm{U}_{\text {trap }}=\frac{1}{2} \mathrm{M}\left(\omega_{\mathrm{x}}^{2} \mathrm{x}^{2}+\omega_{\mathrm{y}}^{2} \mathrm{y}^{2}+\omega_{z}^{2} z^{2}\right) .
$$

The equilibrium position of a trapped ion is $\vec{r}_{0}=\left(\begin{array}{lll}0 & 0 & 0\end{array}\right)$; at this position both electric quadrupole potentials have saddle points and thus both electric quadrupole fields have nulls.

Rydberg ions have giant polarisabilities $\rho$ which scale with the principal quantum number as $n^{7}$. In an electric field of strength $\mathcal{E}$ a Rydberg ion experiences an energy shift due to the quadratic Stark effect

$$
\Delta \mathrm{E}=-\frac{1}{2} \rho \mathcal{E}^{2} .
$$

From Eq. (6.1) the trapping electric field is given by

$$
\overrightarrow{\mathcal{E}}=-\vec{\nabla} \Phi=-2 \alpha \cos \Omega_{\mathrm{rf}} \mathrm{t}\left(\begin{array}{c}
x \\
-\mathrm{y} \\
0
\end{array}\right)+2 \beta\left(\begin{array}{c}
(1+\epsilon) x \\
(1-\epsilon) \mathrm{y} \\
-2 z
\end{array}\right) .
$$

Rydberg excitation is carried out over a much longer timescale than the trap period $\left[\left(\frac{\Omega_{\text {eff }}}{2 \pi}\right)^{-1} \gg\left(\frac{\Omega_{\mathrm{rf}}}{2 \pi}\right)^{-1}=55 \mathrm{~ns}\right.$ in this system] and thus we time-average the squared electric field strength, and use $\alpha^{2} \gg 2 \beta^{2}$ to find

$$
\left\langle\mathcal{E}^{2}\right\rangle \approx 2 \alpha^{2}\left(x^{2}+y^{2}\right) .
$$


And thus in addition to the trap pseudopotential [Eq. (6.4)] the highlypolarisable Rydberg ion experiences the additional harmonic potential

$$
\mathrm{U}_{\mathrm{add}}=-\frac{1}{2} \rho\left\langle\varepsilon^{2}\right\rangle \approx-\rho \alpha^{2}\left(x^{2}+y^{2}\right),
$$

and thus the radial trapping frequencies become

$$
\begin{aligned}
& \omega_{x} \rightarrow \omega_{x}^{\prime} \approx \sqrt{\omega_{x}^{2}-\frac{2 \rho \alpha^{2}}{M}}, \\
& \omega_{y} \rightarrow \omega_{y}^{\prime} \approx \sqrt{\omega_{y}^{2}-\frac{2 \rho \alpha^{2}}{M}},
\end{aligned}
$$

while the axial trapping frequency is still described by Eq. (6.2), because the z-dependence in Eq. (6.7) is negligible.

The energy for Rydberg excitation is thus shifted depending on radial phonon numbers

$$
\Delta E=n_{x} \hbar \Delta \omega_{x}+n_{y} \hbar \Delta \omega_{y},
$$

where

$$
\begin{aligned}
\Delta \omega_{x} & =\omega_{x}^{\prime}-\omega_{x}, \\
\Delta \omega_{y} & =\omega_{y}^{\prime}-\omega_{y},
\end{aligned}
$$

as sketched in Fig. 6.1(a).

The altered trapping pseudopotential also causes phonon-numberchanging transitions to be driven more strongly during Rydberg excitation. This effect is more noticeable when the nulls of the quadrupole trapping fields do not overlap, and it is discussed in Section 6.1.2.

\section{Experimental results}

The dependence of the energy required for Rydberg excitation on the number of radial phonons [Eq. (6.10)] is observed directly by preparing an ion with a known number of radial phonons $\left(n_{x}\right.$ and $\left.n_{y}\right)$ and measuring the Rydberg resonance frequency. The results in Fig. 6.1(b) are consistent with Eq. (6.10). Preparation of phonon-number Fock states is described in Section 3.1.3. 

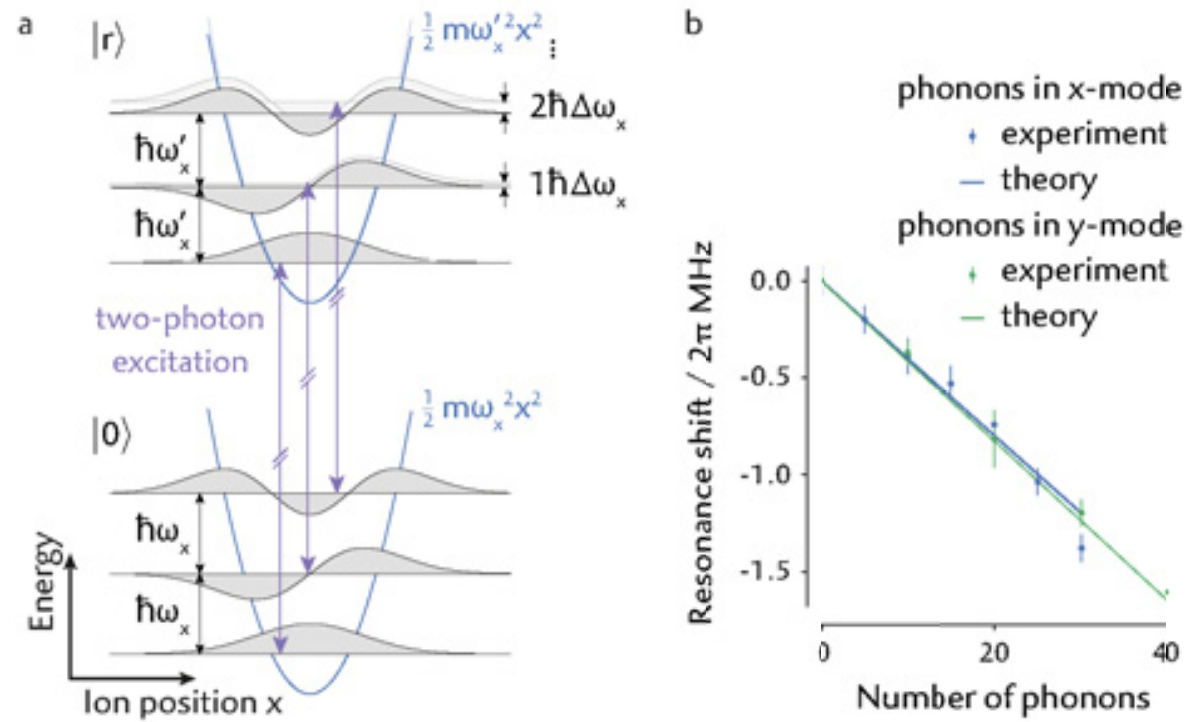

Figure 6.1: Rydberg ions experience an altered trapping potential, and thus the frequency of the Rydberg excitation transition depends linearly on the number of phonons in motional modes. (a) The trapping pseudopotentials are in blue, Rydberg excitation transitions are in purple. (b) Measurement results and theory show excellent agreement; the theory returns $\Delta \omega_{\mathrm{x}}=-2 \pi \times 40 \mathrm{kHz}$ and $\Delta \omega_{y}=-2 \pi \times 41 \mathrm{kHz}$ for $46 \mathrm{~S}_{1 / 2}$ and the trapping parameters used. Error bars are dominated by laser frequency drifts $(68 \%$ confidence interval).

Because of this effect the Rydberg resonance linewidth depends on the widths of the radial phonon distributions. As shown in Fig. 6.2(a), a narrow Rydberg resonance is obtained when a sideband cooled ion (with narrow phonon distributions) is excited, while a broader Rydberg resonance is observed when a Doppler cooled ion (with broader phonon distributions) is excited. The resonance lineshape obtained with a Doppler cooled ion reflects the population distribution over the two radial modes, shown in Fig. 6.2(b). Radial sideband cooling is important for coherent excitation of Rydberg states, because the efficiency of coherent excitation depends on the Rydberg state linewidth (see Section 7.3.2). 


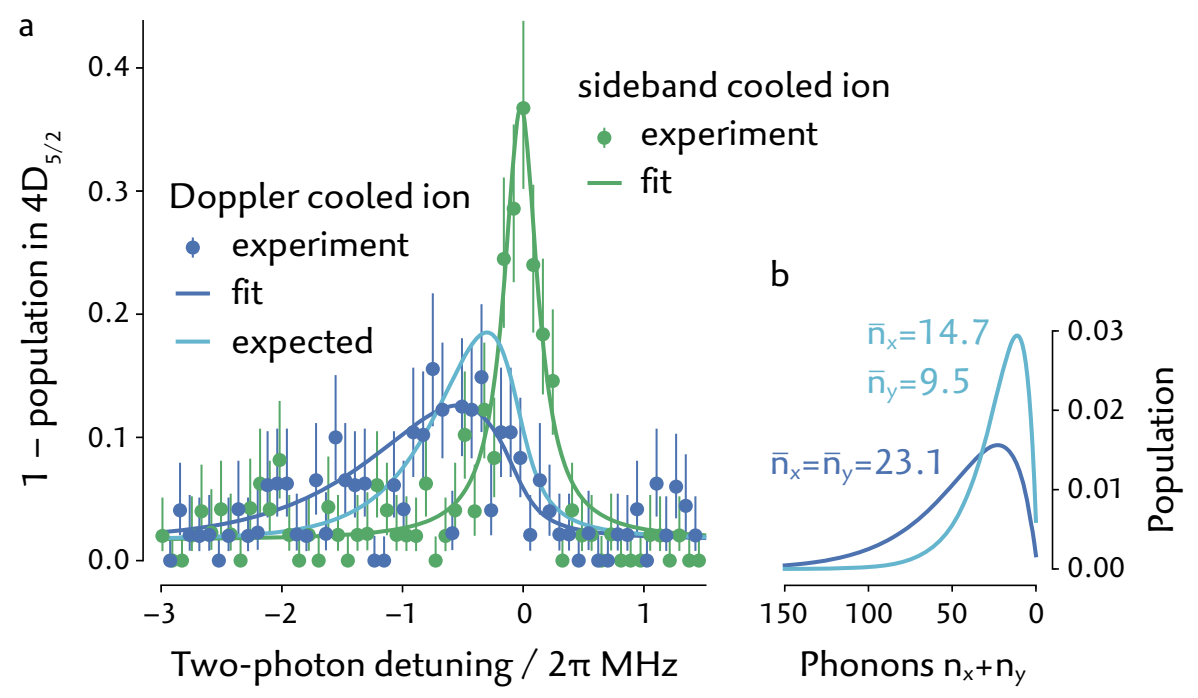

Figure 6.2: (a) Because the Rydberg excitation transition frequency depends on the number of phonons in radial modes, the Rydberg resonance linewidth is broader for a Doppler-cooled ion (with broad phonon distributions) than a sideband-cooled ion (with narrow phonon distributions). The fit of the Doppler cooled ion resonance lineshape reflects thermal phonon distributions with $\bar{n}_{x}=\bar{n}_{y}=23.1 \pm 2.1$. The expected lineshape of the Doppler cooled ion resonance does not match the data as well, it uses independent estimates of $\bar{n}_{x}=14.7 \pm 0.9, \bar{n}_{y}=9.5 \pm 0.8$ and no fit parameters. The $42 S_{1 / 2}$ polarisability and the trap parameters used result in $\Delta \omega_{x} \approx \Delta \omega_{y}=-2 \pi \times 20 \mathrm{kHz}$. Error bars indicate quantum projection noise ( $68 \%$ confidence interval). Distributions of the total number of radial phonons are shown in (b).

\subsubsection{With non-overlapping quadrupole field nulls}

Stray electric fields can cause the electric quadrupole field nulls to be separated [94], and excess micromotion results from this (see Section 3.2.8). When the electric quadrupole field nulls do not overlap additional effects emerge on a trapped Rydberg ion: phonon-numberchanging transitions are driven more strongly during Rydberg excitation, and the energy required for Rydberg excitation depends on the 
distance between the field nulls. These effects stem from the altered trapping pseudopotential of the Rydberg ion.

Before the experimental observation of these effects is shown, the theory underlying these effects is presented.

\section{Background theory}

In this regime the null of the oscillating electric quadrupole field $\left(\vec{r}_{\mathrm{rf}}\right)$ and the null of the static electric quadrupole field $\left(\overrightarrow{\mathrm{r}}_{\mathrm{dc}}\right)$ do not overlap $\left(\vec{r}_{\mathrm{rf}} \neq \overrightarrow{\mathrm{r}}_{\mathrm{dc}}\right)$. Then the equilibrium position of an ion in a low-lying state $\left(\vec{r}_{\mathrm{g}}\right)$ does not correspond to either null $\left(\overrightarrow{\mathrm{r}}_{\mathrm{g}} \neq \overrightarrow{\mathrm{r}}_{\mathrm{rf}}, \overrightarrow{\mathrm{r}}_{\mathrm{dc}}\right)$. Due to the large Rydberg state electric polarisability the equilibrium position of the Rydberg ion $\left(\vec{r}_{R}\right)$ does not overlap with the equilibrium position of an ion in a low-lying state $\left(\vec{r}_{R} \neq \vec{r}_{\mathrm{g}}\right)$.

When the oscillating component of the trapping potential is centred along the $\mathrm{z}$-axis $\overrightarrow{\mathrm{r}}_{\mathrm{rf}}=\left(\begin{array}{lll}0 & 0 & z\end{array}\right)$ and the static trapping potential is centred on $\vec{r}_{\mathrm{dc}}=\left(\begin{array}{lll}x_{\mathrm{dc}} & y_{\mathrm{dc}} & 0\end{array}\right)$ the total electric trapping potential is modified from Eq. (6.1) and becomes

$$
\Phi=\alpha \cos \Omega_{\mathrm{rf}} \mathrm{t}\left(x^{2}-y^{2}\right)-\beta\left[(1+\epsilon)\left(x-x_{\mathrm{dc}}\right)^{2}+(1-\epsilon)\left(y-y_{\mathrm{dc}}\right)^{2}-2 z^{2}\right] .
$$

The oscillating and static electric quadrupole fields (given by $\overrightarrow{\mathcal{E}}=$ $-\vec{\nabla} \Phi)$ then have nulls which do not overlap. The separation between $\vec{r}_{\mathrm{rf}}$ and $\overrightarrow{\mathrm{r}}_{\mathrm{dc}}$ may be introduced by a static electric field

$$
\overrightarrow{\mathcal{E}}_{\text {offset }}=-2 \beta\left(\begin{array}{c}
(1+\epsilon) x_{\mathrm{dc}} \\
(1-\epsilon) y_{\mathrm{dc}} \\
0
\end{array}\right) .
$$

For an ion in a low-lying state the trapping pseudopotential is then centred on $\vec{r}_{g}=\left(\begin{array}{lll}x_{g} & y_{g} & 0\end{array}\right)$ [94]

$$
\mathrm{u}_{\text {trap }}=\frac{1}{2} M\left(\omega_{x}^{2}\left(x-x_{\mathrm{g}}\right)^{2}+\omega_{y}^{2}\left(y-y_{g}\right)^{2}+\omega_{z}^{2} z^{2}\right),
$$


where $^{1}$

$$
\begin{aligned}
& x_{\mathrm{g}}=-\frac{2 e \beta(1+\epsilon) x_{\mathrm{dc}}}{M \omega_{x}^{2}}, \\
& y_{\mathrm{g}}=-\frac{2 e \beta(1-\epsilon) y_{\mathrm{dc}}}{M \omega_{\mathrm{y}}^{2}} .
\end{aligned}
$$

Owing to its high polarisability, a Rydberg ion experiences an additional harmonic potential which is centred on $\vec{r}_{\text {rf }}$ [since the approximation in Eq. (6.7) still holds]

$$
\begin{aligned}
\mathrm{u}_{\text {trap }}^{\prime} & =\mathrm{u}_{\text {trap }}-\frac{1}{2} \rho\left\langle\mathcal{E}^{2}\right\rangle \\
& \approx \mathrm{u}_{\text {trap }}-\rho \alpha^{2}\left(x^{2}+y^{2}\right) .
\end{aligned}
$$

After some simple algebra, we find the trapping pseudopotential of a Rydberg ion is centred on $\vec{r}_{R}=\left(\begin{array}{lll}x_{R} & y_{R} & 0\end{array}\right)$, and it is shifted in energy (relative to the pseudopotential of the ion in a low-lying electronic state) by $\Delta \mathrm{U}$

$$
u_{\text {trap }}^{\prime}=\frac{1}{2} M\left(\omega_{x}^{\prime 2}\left(x-x_{R}\right)^{2}+\omega_{y}^{\prime 2}\left(y-y_{R}\right)^{2}+\omega_{z}^{2} z^{2}\right)+\Delta u,
$$

where

$$
\begin{aligned}
x_{\mathrm{R}} & =x_{\mathrm{g}}\left(1-\frac{2 \rho \alpha^{2}}{M \omega_{x}^{2}}\right)^{-1} \\
y_{\mathrm{R}} & =y_{\mathrm{g}}\left(1-\frac{2 \rho \alpha^{2}}{M \omega_{\mathrm{y}}^{2}}\right)^{-1} \\
\Delta \mathrm{U} & =\frac{1}{2} M\left(\omega_{x}^{2} x_{\mathrm{g}}^{2}+\omega_{y}^{2} y_{\mathrm{g}}^{2}-\omega_{x}^{\prime 2} x_{\mathrm{R}}^{2}-\omega_{y}^{\prime 2} y_{\mathrm{R}}^{2}\right) \\
& \approx-\rho \alpha^{2}\left(x_{\mathrm{g}}^{2}+y_{\mathrm{g}}^{2}\right) \\
& \approx-\rho \alpha^{2}\left(x_{\mathrm{R}}^{2}+y_{\mathrm{R}}^{2}\right)=-\frac{1}{2} \rho\left\langle\mathcal{E}\left(\overrightarrow{\mathrm{r}}_{\mathrm{R}}\right)^{2}\right\rangle
\end{aligned}
$$

and $\omega_{x}^{\prime}$ and $\omega_{y}^{\prime}$ are unchanged from Eq. (6.9). The approximations in Eq. (6.19) are valid when $\frac{2 \rho \alpha^{2}}{M \omega_{x}^{2}} \ll 1$ and $\frac{2 \rho \alpha^{2}}{M \omega_{y}^{2}} \ll 1$. From Eq. (6.19)

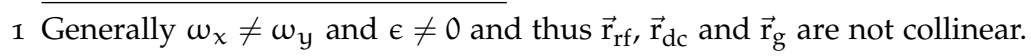


we understand that the energy shift $\Delta \mathrm{U}$ results from the electric field at $\vec{r}_{R}$ acting on the highly-polarisable Rydberg ion.

Due to differences between the equilibrium positions $\vec{r}_{g}$, and $\vec{r}_{R}$ phonon-number-changing transitions may be driven strongly. Rydberg resonance frequency shifts result from $\Delta \mathrm{U}$. These phenomena (which stem from the Rydberg ion polarisability) are presented in the following subsections.

\section{Phonon-number-changing transitions}

The motional components of the total ion wavefunction are described by the eigenstates of the quantum harmonic oscillator. These eigenstates are symmetric about the pseudopotential centre and they depend on the phonon number and the pseudopotential frequency. Due to the difference in trapping pseudopotentials, the radial motional wavefunctions of ions in low-lying electronic states $\left|n_{x}\right\rangle,\left|n_{y}\right\rangle$ and ions in Rydberg states $\left|m_{x}^{\prime}\right\rangle,\left|m_{y}^{\prime}\right\rangle$ do not overlap perfectly $\left\langle m_{x}^{\prime} \mid n_{x}\right\rangle \neq$ $\delta_{m_{x} n_{x}},\left\langle m_{y}^{\prime} \mid n_{y}\right\rangle \neq \delta_{m_{y} n_{y}}$. As a result phonon-number-changing transitions may be driven during Rydberg excitation, with strengths described by the overlap of the motional wavefunctions. The overlap integrals are called Franck-Condon factors. ${ }^{2}$

Phonon-number-changing transitions are expected both when the trapping field nulls overlap and when the nulls do not overlap. When the nulls do not overlap the Franck-Condon factors may be significantly higher and phonon-number-changing transitions may be driven more strongly. This is shown in Fig. 6.3.

In the experiment we introduce an additional static electric field [Eq. (6.13)] so that the saddle points of the electric quadrupole potentials in Eq. (6.12) do not overlap $\left(\vec{r}_{\mathrm{rf}} \neq \overrightarrow{\mathrm{r}}_{\mathrm{dc}}\right)$. This means the pseudopotential centres do not overlap $\left(\vec{r}_{R} \neq \vec{r}_{g}\right)$ and strong phonon-numberchanging transitions are observed in the Rydberg excitation spectrum, shown in Fig. 6.4.

2 Since the Rydberg-excitation lasers counter-propagate (Section 4.4), the contribution to phonon-number-changing transitions described by the Lamb-Dicke parameter is small compared with the Franck-Condon factors considered in this section. 


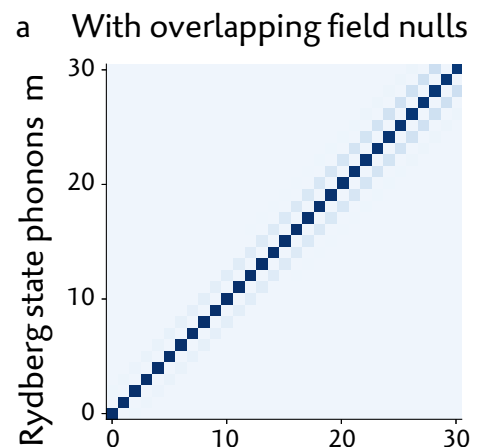

Low-lying state phonons $\mathrm{n}$

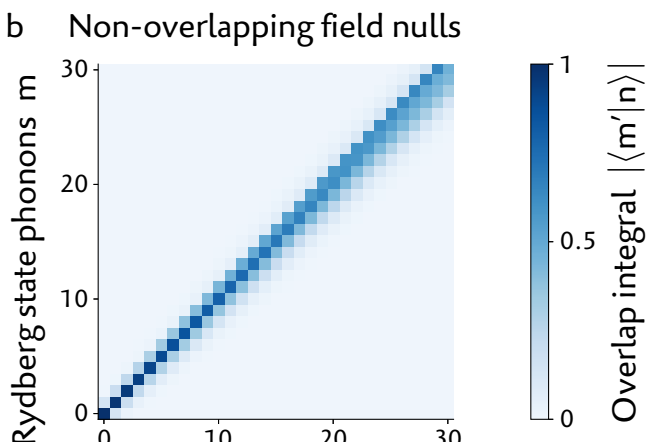

Low-lying state phonons $n$

Figure 6.3: Phonon-number-changing transitions are driven more strongly during Rydberg excitation when the number of phonons is higher and when the field nulls do not overlap. The strength is given by the overlap of motional mode states (Franck-Condon factors). Here a single radial mode is considered, and the trapping frequencies obey $\omega^{\prime}=0.975 \times \omega$. In (a) only transitions which change the phonon-number by an even number are driven, due to symmetry. In (b) the pseudopotential minima of a Rydberg state and a low-lying state are separated by $0.2 \sqrt{\frac{\hbar}{M \omega}}$. During typical operation of the experiment we expect this separation to be $\sim 0.04 \sqrt{\frac{\hbar}{M \omega}}$ as a result of imperfect minimisation of micromotion.

\section{Using $\Delta \mathrm{U}$ to minimise micromotion}

Ion micromotion is introduced in Section 3.2.8. The amplitude of micromotion depends on the separation of the ion equilibrium position and the RF field null $\left|\vec{r}_{\mathrm{g}}-\overrightarrow{\mathrm{r}}_{\mathrm{rf}}\right|$ [94]. Micromotion is minimised by introducing static electric fields such that the distances between $\vec{r}_{\mathrm{rf}}$ and $\vec{r}_{\mathrm{dc}}$ (and thus $\overrightarrow{\mathrm{r}}_{\mathrm{g}}$ and $\overrightarrow{\mathrm{r}}_{\mathrm{R}}$ ) are minimised. The linear Paul trap has two electrodes used to introduce these fields [see Fig. 3.3(b)]. Appropriate compensation fields are found using a range of established techniques, described in Section 3.2.8. Here we introduce a novel method for micromotion minimisation: The separation $\left|\vec{r}_{\mathrm{g}}-\overrightarrow{\mathrm{r}}_{\mathrm{rf}}\right|$ causes a shift in the energy required for Rydberg excitation $\Delta \mathrm{U}$ [see Eq. (6.17)]; by minimising this energy shift, micromotion is minimised. 

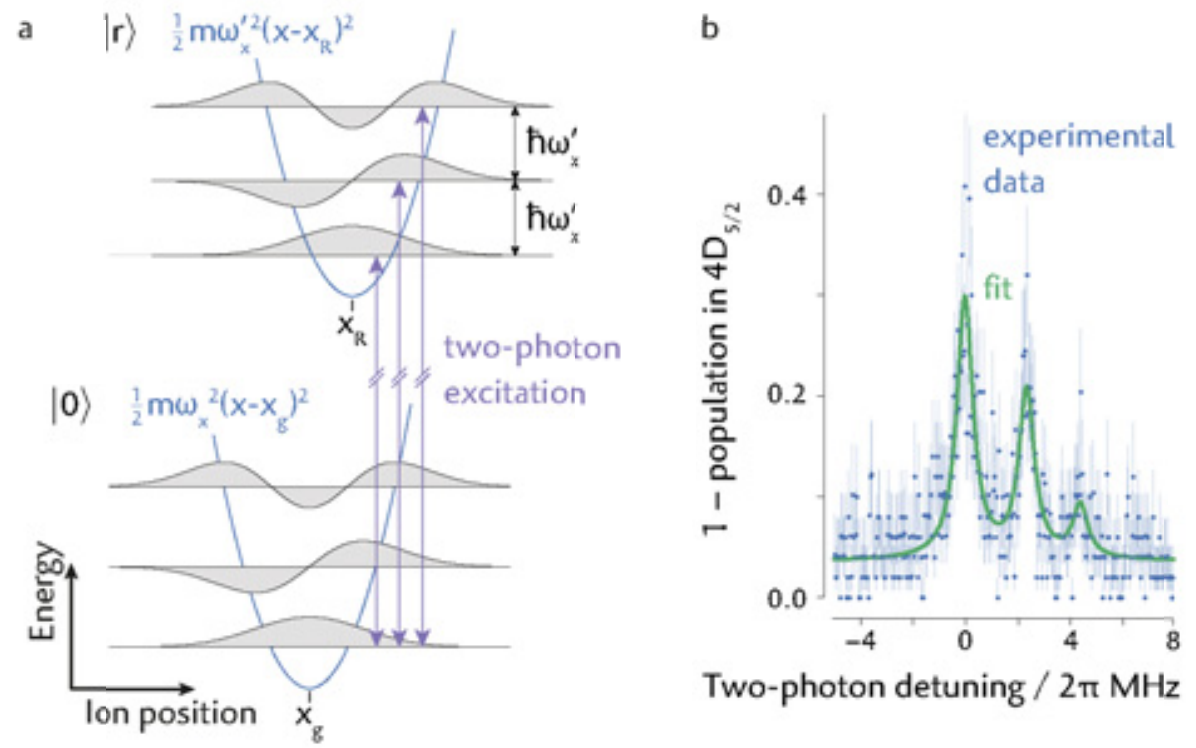

Figure 6.4: Phonon-number-changing transitions due to non-overlapping pseudopotential minima: (a) Because the minima of the trapping pseudopotentials for low-lying states and for Rydberg states do not overlap, the motional modes overlap poorly and phononnumber-changing transitions may be driven strongly during Rydberg excitation. (b) When the field nulls do not overlap the Rydberg resonance structure of a sideband cooled ion shows strong blue sidebands displaced from the carrier transition by $+\omega^{\prime}$ and $+2 \omega^{\prime}$, which correspond to addition of 1 and 2 radial photons. The radial trapping frequencies used are $\omega_{x, y} \approx$ $\omega_{x, y}^{\prime} \approx 2 \pi \times 2.1 \mathrm{MHz}$. Error bars indicate quantum projection noise ( $68 \%$ confidence interval).

This method is demonstrated in Fig. 6.5(a). Here the Rydberg resonance frequency varies quadratically with the voltage applied to a micromotion-compensation electrode. This is consistent with Eq. (6.19), since the distance $\left|\vec{r}_{\mathrm{g}}-\vec{r}_{\mathrm{rf}}\right|$ varies linearly with the voltage [according to Eqs. $(6.13,6.15)]^{3}$

This method for minimising micromotion is limited by the sensitivity of Rydberg states to electric fields and by frequency drifts of the

3 The linear relation holds for one radial direction provided that the ion is close to the trap centre in the orthogonal radial direction. 

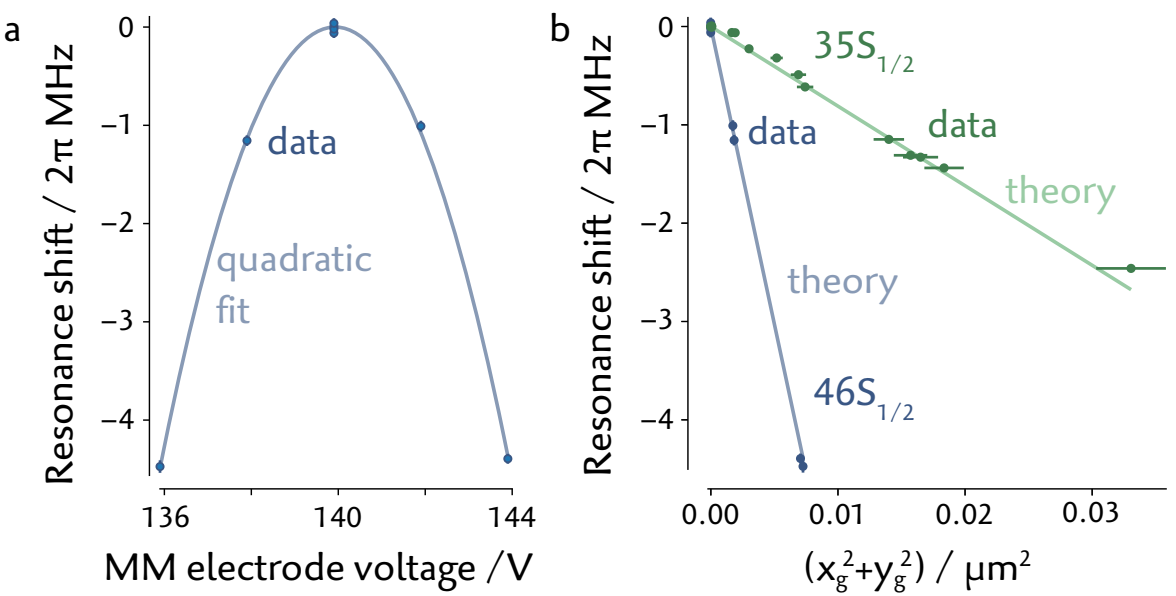

Figure 6.5: The Rydberg resonance frequency depends on the distance between the electric quadrupole field nulls. (a) The Rydberg resonance frequency varies quadratically with the voltages applied to micromotion compensation electrodes. The maximum resonance frequency corresponds to the case with overlapping field nulls (when micromotion is minimised). Here the horizontal micromotion compensation electrode voltage is varied. Error bars are barely discernible on this graph, they result from laser frequency drifts (68\% confidence interval). (b) The Rydberg resonance frequency shift is proportional to the squared distance between the ground state ion equilibrium position and the RF field null, with gradient $-\rho \alpha^{2} / \hbar$ [according to Eq. (6.19)]. The gradient thus depends on the Rydberg state polarisability. The horizontal error bars result from uncertainty in the conversion between voltage and distance (68\% confidence interval).

Rydberg-excitation lasers. Laser frequency drifts $\left(2 \pi \times 300 \mathrm{~Hz} \mathrm{~s}^{-1}\right)$ limit the accuracy with which resonance frequencies are determined and thus the accuracy with which $\Delta \mathrm{U}$ is determined to $\sim 2 \pi \times$ $100 \mathrm{kHz}$. Using Rydberg state $46 \mathrm{~S}_{1 / 2}$ (with polarisability $\rho_{46 \mathrm{~S}}=$ $6 \times 10^{-31} \mathrm{C} \mathrm{m}^{2} \mathrm{~V}^{-1}$ ) the residual oscillating electric field strength at the equilibrium position of the ion $\vec{r}_{\mathrm{g}}$ is reduced to $\varepsilon_{\mathrm{rf}} \sim 20 \mathrm{Vm}^{-1}$. With the best micromotion minimisation techniques the residual field strength can be reduced to $\varepsilon_{\mathrm{rf}} \sim 0.3 \mathrm{~V} \mathrm{~m}^{-1}$ [95]. 
This method may allow significantly lower residual field strengths to be reached if the frequency stability of the UV lasers was improved and if Rydberg states with higher polarisabilities were used. With laser frequency drifts $<2 \pi \times 1 \mathrm{~Hz} \mathrm{~s}^{-1}$ (this is experimentally realisable) $\Delta \mathrm{U}$ may be determined with precision $\sim \hbar 2 \pi \times 1 \mathrm{kHz}$ (provided the Rydberg state resonance is narrow enough). $\mathrm{nP}_{1 / 2}$ states have polarisabilities $\sim 5$ times higher than $\mathrm{nS}_{1 / 2}$ states and have been excited in our lab (see the Outlook in Chapter 8). State $59 \mathrm{P}_{1 / 2}$ has linewidth $\Gamma \approx 2 \pi \times 2 \mathrm{kHz}$ at $300 \mathrm{~K}$ and polarisability $\rho \approx-10^{10} \mathrm{C}^{2} \mathrm{~V}^{-1}$. By using $59 \mathrm{P}_{1 / 2}$ as a probe and with more stable frequency references residual field strengths may be reduced to $\varepsilon_{\mathrm{rf}} \sim 0.3 \mathrm{~V} \mathrm{~m}^{-1}$.

In Fig. 6.5(b) we confirm Eq. (6.19) is followed quantitatively. The voltage axis in Fig. 6.5(a) is converted to a distance in Fig. 6.5(b) using the EMCCD camera image of a trapped ion. This method relies on knowledge of the image magnification, which is measured using two ions as described in Section 5.2.1.

\subsection{EFFECTS ON RYDBERG IONS WITH LARGE QUADRUPOLE MO- MENTS}

Rich resonance structures are observed when Rydberg D-states are excited. The structures include Floquet sidebands, which result from coupling between levels driven by the time-dependent electric quadrupole trapping field. The strength of this coupling depends on the electric quadrupole moments of the states in question; effects of the coupling are best observed with $J>\frac{1}{2}$ states (such as $\mathrm{nD}_{3 / 2}$ ) which have sizeable quadrupole moments.

The coupling is negligible for $J=\frac{1}{2}$ states $\left(\mathrm{nS}_{1 / 2}\right.$ and $\left.n \mathrm{P}_{1 / 2}\right)$ with $\mathrm{n}<50$ [49], since $\mathrm{J}=\frac{1}{2}$ states have negligible quadrupole moments and the energy separation between $J=\frac{1}{2}$ and $J>\frac{1}{2}$ states makes coupling between them weak. The experiments proposed in Section 1.3.1 are concerned with Rydberg $S_{1 / 2}$ and $P_{1 / 2}$-states because they are simpler systems.

The theory behind the trap-induced coupling is presented in Section 6.2.1. The observed effects of this coupling are understood in 
terms of Floquet theory; this theory is introduced in Section 6.2.2. Experimental results are shown in Section 6.2.3.

\subsubsection{Theory behind the trap-induced coupling}

The Rydberg electron interacts with the trapping electric quadrupole fields according to

$$
\begin{aligned}
\mathrm{H}_{\mathrm{eQ}} & =-e \Phi \\
& =e \beta\left(x^{2}+y^{2}-2 z^{2}\right)-e \alpha \cos \Omega_{\mathrm{rf}} \mathrm{t}\left(x^{2}-y^{2}\right) \\
& =-4 \sqrt{\frac{\pi}{5}} e r^{2} \beta Y_{2}^{0}-2 \sqrt{\frac{2 \pi}{15}} \operatorname{er}^{2} \alpha \cos \Omega_{\mathrm{rf}} \mathrm{t}\left(Y_{2}^{2}+Y_{2}^{-2}\right) \\
& =\sum_{\mathrm{q}} \underbrace{A_{\mathrm{q}}}_{\begin{array}{c}
\text { E-field } \\
\text { gradient }
\end{array}} \times \underbrace{\mathrm{er}^{2} Y_{2}^{\mathrm{q}}}_{\begin{array}{c}
\text { spherical electric } \\
\text { quadrupole moment }
\end{array}},
\end{aligned}
$$

where for clarity we define

$$
A_{o}=-4 \sqrt{\frac{\pi}{5}} \beta, \quad A_{ \pm 2}=-2 \sqrt{\frac{2 \pi}{15}} \alpha \cos \Omega_{\mathrm{rf}} \mathrm{t} .
$$

We use the trapping potential from Eq. (6.1) and neglect the trap anisotropy $\epsilon$. By writing $\mathrm{H}_{\mathrm{eQ}}$ in terms of spherical harmonics we see $\mathrm{H}_{\mathrm{eQ}}$ is composed of spherical electric quadrupole moments and electric field gradients.

For Rydberg D-states the strength of $\mathrm{H}_{\mathrm{eQ}}$ (which scales as $\mathrm{n}^{4}$ ) is much weaker than the fine structure splitting $\left(\sim n^{-3}\right)$ until $n \approx 45$. We experimentally investigate $24 \mathrm{D}_{3 / 2}$ and $27 \mathrm{D}_{3 / 2}$, and thus to describe the results we need only consider the manifold of states described by quantum numbers $\{n, L, S, J\}$ which have different $m_{J}$. 
In our experiment the trap axis coincides with the quantisation axis (defined by the magnetic field, see Section 3.2.6) and the matrix elements of the components of $\mathrm{H}_{\mathrm{eQ}}$ are

$$
\begin{aligned}
& \left\langle\mathrm{nLSJm} \mathrm{m}_{\mathrm{J}}^{\prime}\left|A_{\mathrm{q}} \mathrm{er}^{2} Y_{2}^{\mathrm{q}}\right| \mathrm{nLSJm} \mathrm{J}\right\rangle \\
& =A_{\mathrm{q}} e\left\langle\mathrm{nLJ}\left|\mathrm{r}^{2}\right| \mathrm{nLJ}\right\rangle\left\langle\mathrm{LSJm}_{\mathrm{J}}^{\prime}\left|\mathrm{Y}_{2}^{\mathrm{q}}\right| \mathrm{LS} \mathrm{Lm}_{\mathrm{J}}\right\rangle \\
& =(-1)^{J-m_{J}^{\prime}} A_{q} e\left\langle r^{2}\right\rangle\left(L S J\left\|Y_{2}\right\| L S J\right)\left(\begin{array}{ccc}
J & 2 & J \\
-m_{J}^{\prime} & q & m_{J}
\end{array}\right) \\
& =(-1)^{J-m_{j}^{\prime}+1} \frac{1}{2} \sqrt{\frac{5}{\pi}} A_{q} \Theta(n L S J) \\
& \times\left(\begin{array}{ccc}
J & 2 & J \\
-J & 0 & J
\end{array}\right)^{-1}\left(\begin{array}{ccc}
J & 2 & J \\
-m_{J}^{\prime} & q & m_{J}
\end{array}\right) .
\end{aligned}
$$

In Eq. (6.25) we make use of the separability of the atomic wavefunction and in Eqs. $(6.26,6.27)$ the Wigner-Eckard theorem is used. The electric quadrupole moment of $|n L S J\rangle$ is defined by the diagonal matrix element of the state with $m_{J}=J[131]$

$$
\begin{aligned}
\Theta(n L S J) & =-\frac{e}{2}\left\langle n L S J J\left|3 z^{2}-r^{2}\right| n L S J J\right\rangle \\
& =-2 \sqrt{\frac{\pi}{5}} e\left\langle r^{2}\right\rangle\left\langle S L J J\left|Y_{2}^{0}\right| S L J J\right\rangle \\
& =-2 \sqrt{\frac{\pi}{5}} e\left\langle r^{2}\right\rangle\left(S L J J\left\|Y_{2}\right\| S L J J\right)\left(\begin{array}{ccc}
J & 2 & J \\
-J & 0 & J
\end{array}\right) .
\end{aligned}
$$

Whenever the trap axis and the quantisation axis do not coincide, the matrix elements of $\mathrm{H}_{\mathrm{eQ}}$ are found by rotating between the bases set by the trap axis and the quantisation basis. In the experiment the axes coincide and so we do not need to rotate between bases here.

Selection rules become apparent through the properties of Wigner3j symbols. The electric quadrupole moment $\Theta(n L S J)$ and the matrix elements of Eq. (6.27) are zero when the triangle inequality $|J-2| \leqslant J$ is not satisfied, i.e. for $J=\frac{1}{2}$ states. This is why we do not observe quadrupole effects in the excitation spectra of Rydberg $S_{1 / 2}$-states. Non-zero matrix elements must satisfy $m_{J}^{\prime}=m_{J}+q$ and thus the $\mathrm{q}=0$ term has only on-diagonal elements which cause levels to be 
shifted in energy, while the $q= \pm 2$ terms have only off-diagonal elements which couple states which differ in $m_{J}$ by 2 . The coupling oscillates with frequency $\Omega_{\mathrm{rf}}$.

Hamiltonian $\mathrm{H}_{\mathrm{eQ}}$ is not only relevant in trapped Rydberg ion experiments; trapped ion atomic clocks must account for quadrupole shifts in transitions frequencies which result from $\mathrm{H}_{\mathrm{eQ}}$ [131]. Perturbation theory is used to describe these shifts of $\sim 2 \pi \times 10 \mathrm{~Hz}$. The effects of $\mathrm{H}_{\mathrm{eQ}}$ are more dramatic in Rydberg states because $\Theta(n L S J)$ scales with $\mathrm{n}^{4}$, for example $\Theta\left(27 \mathrm{D}_{3 / 2}\right)=5.3 \times 10^{4}$ e $_{0}{ }^{2}$ (calculated using theoretical wavefunctions, see Section 2.2), while $\Theta\left(4 D_{5 / 2}\right)=3.0$ e $a_{0}{ }^{2}$ [132] and $\Theta\left(4 \mathrm{D}_{3 / 2}\right)=2.0 \mathrm{e} \mathrm{a}_{0}^{2}$ [133].

The $q=0$ term of $H_{e Q}$ causes the Zeeman sublevels of a $n D_{3 / 2}$ state to be shifted according to

$$
E_{s}=(-1)^{\left|m_{J}\right|+1 / 2} 2 \beta \Theta\left(n_{3 / 2}\right) .
$$

This formula holds for a single trapped ion in the absence of micromotion. When multiple ions are trapped the Coulombic fields contribute to the static electric quadrupole fields [9o].

The $\mathrm{q}= \pm 2$ components of $\mathrm{H}_{\mathrm{eQ}}$ can be written as

$$
\begin{array}{r}
\mathrm{H}_{\mathrm{rf}}=\hbar C \cos \Omega_{\mathrm{rf}} \mathrm{t} \sum_{m_{J}=1 / 2}^{3 / 2}\left|n D_{3 / 2}\left(m_{J}-2\right)\right\rangle\left\langle\mathrm{nD}_{3 / 2} \mathrm{~m}_{J}\right| \\
+\left|n D_{3 / 2}\left(m_{J}+2\right)\right\rangle\left\langle\mathrm{nD}_{3 / 2} m_{J}\right|,
\end{array}
$$

where

$$
C=\frac{2 \alpha \Theta\left(n D_{3 / 2}\right)}{\sqrt{3} \hbar} .
$$

The two effects are represented in Fig. 6.6.

When we excite $24 \mathrm{D}_{3 / 2}$ and $27 \mathrm{D}_{3 / 2}$ Rydberg states in the laboratory we observe rich resonance structures containing Floquet sidebands. The sidebands appear because the coupling strength $C$ is comparable with the trap drive $\Omega_{\mathrm{rf}}$ and thus effects of $\mathrm{H}_{\mathrm{rf}}$ extend beyond what may be described within the rotating wave approximation. Floquet theory is introduced next, before the experimental results are shown in Section 6.2.3. 


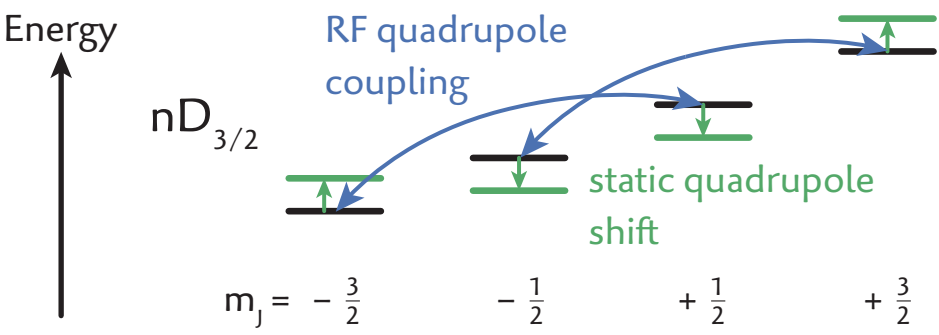

Figure 6.6: The static electric quadrupole field shifts the energies of Rydberg $\mathrm{D}_{3 / 2}$-state sublevels. When the trap axis and magnetic field are collinear the RF oscillating field couples next-neighbouring sublevels. In the absence of trap effects sublevels are split because of the Zeeman effect.

\subsubsection{Floquet theory}

The energy spectrum of a periodically-driven system, with drive frequency $\Omega$, shows sidebands at integer multiples of $\hbar \Omega$. These sidebands may be understood in terms of Floquet theory, which is outlined here following the approach in [134].

The Hamiltonian of a periodically-driven system satisfies

$$
H(\vec{r}, t)=H(\vec{r}, t+T),
$$

where $T=\frac{2 \pi}{\Omega}$. The periodicity of $H$ suggests looking for solutions of the time-dependent Schrödinger equation $\psi(\vec{r}, t)$ with the same periodicity (to within a phase factor)

$$
\psi(\vec{r}, t)=e^{-i \epsilon t / \hbar} \phi_{\epsilon}(\vec{r}, t),
$$

where

$$
\phi_{\epsilon}(\vec{r}, t)=\phi_{\epsilon}(\vec{r}, t+T) .
$$

By substituting Eq. (6.35) into the time-dependent Schrödinger equation $\mathrm{H} \psi=i \hbar \frac{\partial \psi}{\partial t}$ we see

$$
\left(H-i \hbar \frac{\partial}{\partial t}\right) \phi_{\epsilon}=\epsilon \phi_{\epsilon}
$$


and thus $\phi_{\epsilon}$ is an eigenstate of

$$
\mathcal{H}=\mathrm{H}-i \hbar \frac{\partial}{\partial \mathrm{t}} \text {. }
$$

The eigenvalue $\epsilon$ is called a quasi-energy and the solution of Eq. (6.37) is a quasi-energy state or Floquet state. For each eigenstate $\phi_{\epsilon}$ of $\mathcal{H}$ with eigenvalue $\epsilon$ there is a family of eigenstates $\phi_{\epsilon} e^{i k \Omega t}$ which belong to the same Floquet state. The states have eigenvalues $\epsilon+k \hbar \Omega$ and $k$ takes integer values.

Floquet states form a convenient basis for modelling a periodicallydriven system; the time-dependence of the drive is transferred onto the basis states, such that the coupling between Floquet states is timeindependent. This means methods for solving the time-independent Schrödinger equation or Liouville equation may be used. The downside is that the dimension of a system is larger in the Floquet basis than in the bare state basis [135].

The Floquet basis may be used to gain insight into a periodicallydriven system. In Fig. 6.7 a manifold of Rydberg $\mathrm{nD}_{3 / 2}$ states is expanded in the Floquet basis and the coupling of Eq. (6.32) is represented. The difference in quasi-energy between coupled levels is given

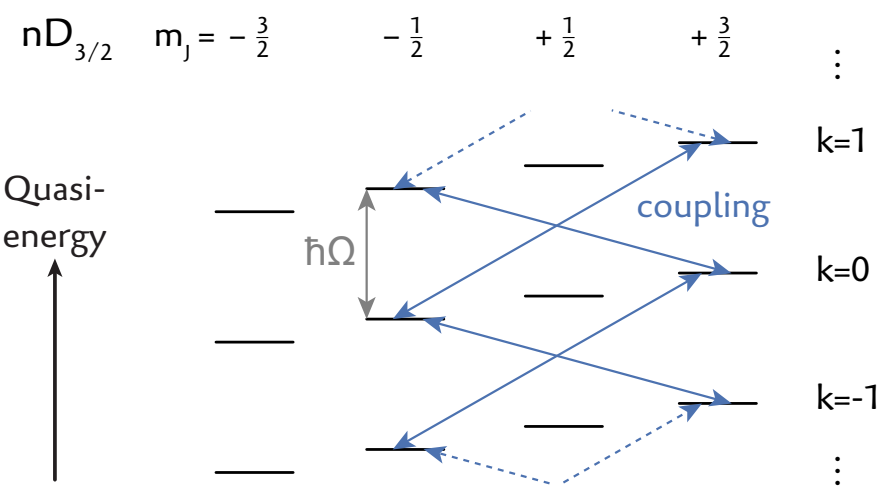

Figure 6.7: Quadrupole field-driven coupling between levels shown in the Floquet basis: $k$ is an index which labels Floquet modes, states with $\Delta \mathrm{m}_{\mathrm{J}}=2$ and $\Delta \mathrm{k}=1$ are coupled. For clarity only coupling between $m_{J}=-\frac{1}{2}$ and $\frac{3}{2}$ states is shown.

by both $\hbar \Omega_{\mathrm{rf}}$ and the Zeeman splitting of the levels. As a result the excitation spectra of Rydberg $D_{3 / 2}$-states include Floquet sidebands 
which are positioned at $\pm \Omega_{\text {rf }}$ relative to next-neighbouring Zeeman sublevels $\left(\Delta \mathrm{m}_{\mathrm{J}}= \pm 2\right)$.

\subsubsection{Experimental results}

The coupling between Rydberg states driven by the oscillating quadrupole field is observed in the excitation spectrum of $27 \mathrm{D}_{3 / 2}$. The ion is prepared in a mixture of $4 \mathrm{D}_{3 / 2}$ sublevels. The data shown in Fig. 6.8(a) is recorded using UV laser light which drives the twophoton transitions $4 \mathrm{D}_{3 / 2}, \mathrm{~m}_{\mathrm{J}}=\frac{3}{2} \leftrightarrow 27 \mathrm{D}_{3 / 2}, \mathrm{~m}_{\mathrm{J}}=\frac{3}{2}$ and $4 \mathrm{D}_{3 / 2}, \mathrm{~m}_{\mathrm{J}}=$ $\frac{1}{2} \leftrightarrow 27 \mathrm{D}_{3 / 2}, \mathrm{~m}_{\mathrm{J}}=\frac{1}{2}$. These transitions are selected by using appropriate polarisations of the UV laser beams (see Section 4.5). The excitation spectra corresponding to these two transitions overlap, this is because neither transition is affected by the Zeeman effect, and because both Rydberg states couple to the next-neighbouring sublevel with $\Delta m_{\mathrm{J}}=-2$.

Spectra are measured using two different strengths of trapping fields; Floquet sidebands are observed in both cases. When stronger trapping fields are used the coupling $C$ between the levels is higher and the sidebands are more pronounced. The sidebands appear near to multiples of the frequency of the oscillating trapping electric field $\Omega_{\mathrm{rf}}=2 \pi \times 18.2 \mathrm{MHz}$.

$\mathrm{H}_{\mathrm{rf}}$ couples states with $\Delta \mathrm{m}_{\mathrm{J}}= \pm 2$. As shown in Fig. 6.8(b), the firstorder sidebands are offset from the next-neighbouring Zeeman levels by $\pm \Omega_{\mathrm{rf}}$ and thus the offset from the carrier transition is $\left( \pm \Omega_{\mathrm{rf}}-2 \pi \times\right.$ $6.5 \mathrm{MHz}$ ) in the magnetic field of strength $B=0.29 \mathrm{mT}$. The secondorder sidebands are offset from the carrier transition by $\pm 2 \Omega_{\mathrm{rf}}$.

Using the theory value of the quadrupole moment $\Theta\left(27 \mathrm{D}_{3 / 2}\right)=$ $5.3 \times 10^{4} \mathrm{e} \mathrm{a}_{0}{ }^{2}$ (see Section 2.2) and Eqs. $(6.31,6.33$ ) we can estimate the coupling strength $C$ and the energy shift $\left|E_{s}\right|$ : With relatively strong trapping fields (field gradients $\alpha=8.2 \times 10^{8} \mathrm{~V} \mathrm{~m}^{-2}$ and $\beta=$ $\left.6.8 \times 10^{6} \mathrm{~V} \mathrm{~m}^{-2}\right) \mathrm{C}=2 \pi \times 34 \mathrm{MHz}$ and $\left|\mathrm{E}_{s}\right| / \hbar=2 \pi \times 490 \mathrm{kHz}$. With weaker trapping fields $\left(\alpha=3.3 \times 10^{8} \mathrm{~V} \mathrm{~m}^{-2}\right.$ and $\left.\beta=5.7 \times 10^{5} \mathrm{~V} \mathrm{~m}^{-2}\right)$ $\mathrm{C}=2 \pi \times 14 \mathrm{MHz}$ and $\left|\mathrm{E}_{s}\right| / \hbar=2 \pi \times 41 \mathrm{kHz}$. Floquet sidebands emerge because $C \nless \Omega_{\text {rf }}$. The energy shifts $\left|E_{s}\right|$ are not resolved. 


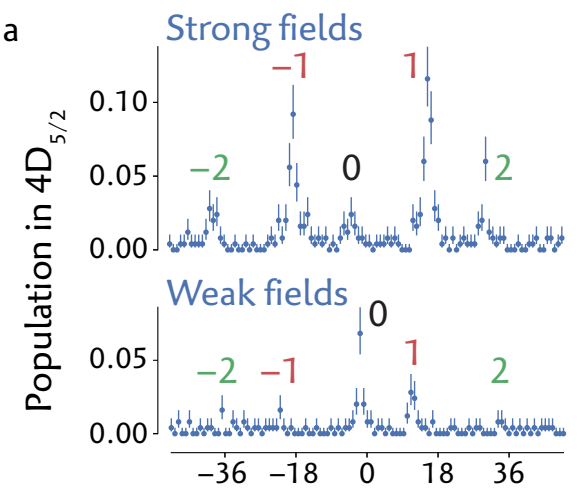

Two-photon detuning / $2 \pi \mathrm{MHz}$

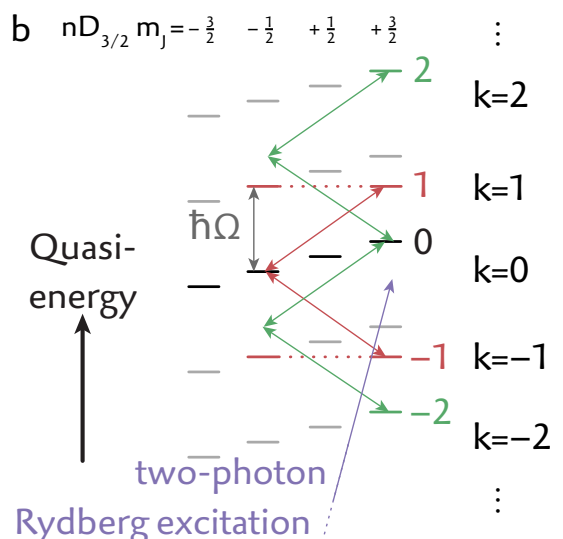

Rydberg excitation

Figure 6.8: (a) The oscillating quadrupole trapping field couples the $27 \mathrm{D}_{3 / 2}$ sublevels, this results in Floquet sidebands in $27 \mathrm{D}_{3 / 2}$ excitation spectra. The trapping field oscillates at $\Omega_{\mathrm{rf}}=2 \pi \times 18.2 \mathrm{MHz}$; first-order Floquet sidebands are observed around $2 \pi \times 18 \mathrm{MHz}$ from the carrier resonance, second-order sidebands are observed around $2 \pi \times 36 \mathrm{MHz}$ from the carrier. The sidebands are stronger when stronger trapping fields are used. The frequency axis has uncertainty $\approx 2 \pi \times 2 \mathrm{MHz}$ in the centre frequency and $\sim 10 \%$ uncertainty in a frequency range, as explained in the body text. Error bars indicate quantum projection noise (68\% confidence interval). (b) The relevant trap-induced coupling for state $n D_{3 / 2}, m_{J}=\frac{3}{2}$ is shown in the Floquet basis. First-order Floquet sidebands (red) result from coupling to the $m_{\mathrm{J}}=-\frac{1}{2}$ sublevel. Second-order sidebands (green) result from coupling first to the $m_{J}=-\frac{1}{2}$ sublevel then to the $m_{J}=\frac{3}{2}$ sublevel.

The frequency axis in Fig. 6.8(a) has uncertainty $\approx 2 \pi \times 2 \mathrm{MHz}$ in the centre frequency and $\sim 10 \%$ uncertainty in a frequency range. These uncertainties result because of the method used to scan the $307 \mathrm{~nm}$ laser frequency: The frequency was scanned by changing the length of the $615 \mathrm{~nm}$ laser frequency reference using piezoelectric rings (Section 3.3.4); the piezoelectric rings respond linearly only to a first approximation, they also show hysteresis effects. The centre frequency is estimated by measuring excitation spectra between other sublevels of $4 \mathrm{D}_{3 / 2}$ and $27 \mathrm{D}_{3 / 2}$ using different UV laser light polarisations, then taking the average centre frequency of all the excitation 
spectra. Further data has been collected since introduction of the AOM used to scan the $307 \mathrm{~nm}$ laser frequency. This data is published in [130] and shows excitation spectra of $24 \mathrm{D}_{3 / 2}$. The spectra are, however, less easy to interpret because of ac-Stark shifts. 

In neutral Rydberg atom systems a host of quantum mechanical phenomena have been investigated, several of which are reviewed in Section 1.2. Quantum mechanical phenomena are observed most easily in isolated systems with strong coupling between only a few levels. With our understanding of Rydberg ion trap effects (see Chapter 6) we isolate a single Rydberg level and couple it with two other atomic levels using light fields; we then investigate the quantum-mechanical phenomena which appear.

While coupling between two levels gives rise to Rabi oscillations, coupling between the levels of a three-level system results in a range of phenomena [136], including the Autler-Townes effect (reported in Section 7.2), electromagnetically-induced transparency (EIT), twophoton Rabi oscillations (Section 7.3), as well as stimulated Raman adiabatic passage (STIRAP) (Section 7.4). Using STIRAP for coherent Rydberg excitation and deexcitation we measure the Rydberg state lifetime (Section 7.4.2) and carry out a single-qubit Rydberg phase gate (Section 7.4.3). This gate demonstrates the basic workings of a Rydberg ion quantum computer. Many of the results in this chapter are published in Physical Review Letters [137].

Before describing the results, the three-level system is introduced in Section 7.1.

\subsection{THE THREE-LEVEL SYSTEM}

A single Rydberg level is coupled to two other atomic levels using two UV laser fields. To isolate a single Rydberg level we avoid trap-driven coupling between Rydberg states (Section 6.2) by using a Rydberg S-state and we mitigate effects of the trap on the highly-polarisable Rydberg state (Section 6.1) by employing radial sideband cooling and by minimising micromotion. 
The three levels coupled by light fields are $|0\rangle \equiv 4 \mathrm{D}_{5 / 2}, \mathrm{~m}_{\mathrm{J}}=-\frac{5}{2}$, $|e\rangle \equiv 6 \mathrm{P}_{3 / 2}, \mathrm{~m}_{\mathrm{J}}=-\frac{3}{2}$ and $|\mathrm{r}\rangle \equiv \mathrm{nS}_{1 / 2}, \mathrm{~m}_{\mathrm{J}}=-\frac{1}{2}$. A sublevel of $4 D_{5 / 2}$ is used rather than a sublevel of $4 D_{3 / 2}$ since population may be initialised in a single $4 \mathrm{D}_{5 / 2}$ sublevel (Section 3.1.3). The levels are in a ladder configuration, as shown in Fig. 7.1.

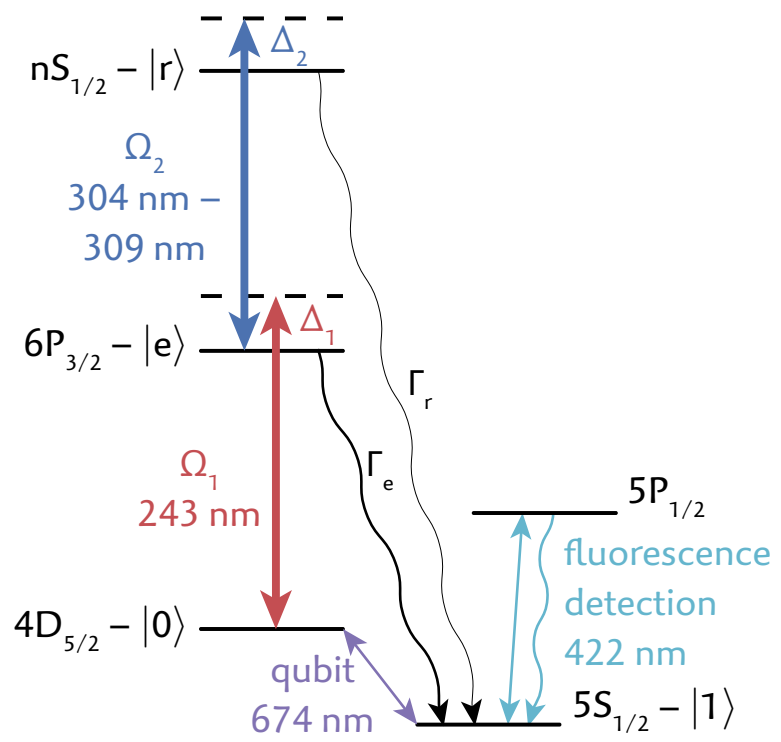

Figure 7.1: Three atomic levels of ${ }^{88} \mathrm{Sr}^{+}$are coupled by two UV laser fields. Population in $|e\rangle$ or $|r\rangle$ decays mostly to $5 S_{1 / 2}$; detection of scattered $422 \mathrm{~nm}$ light heralds excitation from $|0\rangle$ and decay to $5 S_{1 / 2}$. A $4 D_{5 / 2}$ sublevel and a $5 S_{1 / 2}$ sublevel are used to store a qubit.

Within the rotating wave approximation the coupling Hamiltonian describing the light-matter interaction is given by

$$
H_{c}=\frac{\hbar}{2}\left(\begin{array}{ccc}
0 & \Omega_{1} & 0 \\
\Omega_{1} & 2 \Delta_{1} & \Omega_{2} e^{i \phi} \\
0 & \Omega_{2} e^{-i \phi} & 2 \Delta_{1}+2 \Delta_{2}
\end{array}\right)
$$

in the basis $\{|0\rangle,|e\rangle,|r\rangle\} . \Omega_{1}, \Omega_{2}, \Delta_{1}$ and $\Delta_{2}$ are the Rabi frequencies and detunings from resonance of the first and second Rydberg excitation laser fields, and $\phi$ is the relative phase of the Rydberg excitation 
laser fields within the rotating frame. The lifetimes and decay rates of $|e\rangle$ and $|r\rangle$ are given by $\tau_{e}, \Gamma_{e}$ and $\tau_{r}, \Gamma_{r}$.

\subsection{AUTLER-TOWNES EFFECT}

When two levels of a three-level system are coupled by an intense field, the absorption peak of a transition involving the third level, probed by a weak field, is split [138]. This phenomenon is called the Autler-Townes effect.

The first Rydberg excitation laser field probes the $|0\rangle \leftrightarrow|e\rangle$ transition while the second Rydberg excitation laser field couples $|e\rangle$ and $|r\rangle$. The $|0\rangle \leftrightarrow|e\rangle$ resonance lineshape is observed by scanning the frequency of the probe laser across the resonance, with $\Omega_{1} \ll \Gamma_{e}$, as is described in Section 4.1.1. When the $|e\rangle$ and $|r\rangle$ levels are strongly coupled $\left(\Omega_{2}>\Gamma_{e}, \Gamma_{r}\right)$, the absorption profile of the probe field shows two peaks. When the coupling field is resonant with the $|e\rangle \leftrightarrow|\mathrm{r}\rangle$ transition $\left(\Delta_{2}=0\right)$ the splitting of the peaks is given by the coupling field Rabi frequency $\Omega_{2}$. This effect is shown in Fig. 7.2.

When $\Omega_{2}>\Gamma_{e} \gg \Omega_{1}$ the eigenstates and eigenvalues of Eq. (7.1) are

$$
\begin{array}{ll}
\left|\phi_{0}\right\rangle=|0\rangle & \mathrm{E}_{0}=0 \\
\left|\phi_{ \pm}\right\rangle=\frac{-\Delta_{2} \pm \sqrt{\Delta_{2}^{2}+\Omega_{2}^{2}}}{\Omega_{2}}|e\rangle+|\mathrm{r}\rangle & \mathrm{E}_{ \pm}=\frac{\hbar}{2}\left(\Delta_{2} \pm \sqrt{\Delta_{2}^{2}+\Omega_{2}^{2}}\right) .
\end{array}
$$

$\left|\phi_{0}\right\rangle$ is the bare atomic state $|0\rangle,\left|\phi_{ \pm}\right\rangle$are light-dressed states. When $\Delta_{2}=0$ the dressed eigenstates simplify to $\left|\phi_{ \pm}\right\rangle=|e\rangle \pm|r\rangle$ and the difference between the eigenenergies becomes $E_{+}-E_{-}=\hbar \Omega_{2}$. Thus the resonance peaks in Fig. 7.2 correspond to excitation of the dressed states $|e\rangle \pm|r\rangle$, split by $\Omega_{2}$. When $\left|\Delta_{2}\right| \gg \Omega_{2}$ the eigenenergies $E_{ \pm}$take the familiar form of the ac-Stark shift.

By fitting a model of the resonance structure to the experimental data we can extract $\Omega_{2}$ and $\Delta_{2}$. The model is derived by including decay channels from $|e\rangle$ and $|r\rangle$ to the $5 S_{1 / 2}$ state. The derivation involves adiabatic elimination of $|e\rangle$ and $|r\rangle$, which is justified as follows: The decay rates of the dressed states are of similar magnitudes as the 


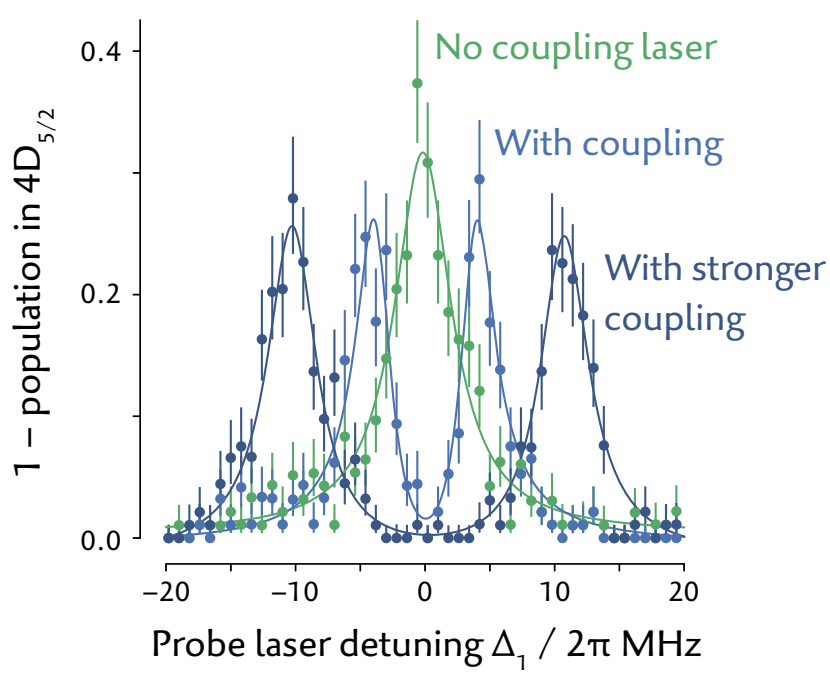

Figure 7.2: Autler-Townes splitting in the three-level system. The ion is prepared in $|0\rangle$ and illuminated by the probe laser light. The Lorentzian absorption profile of $|0\rangle \leftrightarrow|e\rangle$ is observed when the coupling laser field is switched off. When the resonant coupling field is switched on an Autler-Townes doublet emerges, with splitting $\Omega_{2}$. The solid lines are fits to the data; the fits return $\Omega_{2}=2 \pi \times(7.88 \pm 0.25) \mathrm{MHz}$ for the blue data points and $\Omega_{2}=2 \pi \times(20.9 \pm 0.4) \mathrm{MHz}$ for the dark blue data points. Here $|r\rangle=42 S_{1 / 2}, m_{J}=-\frac{1}{2}$. Error bars indicate quantum projection noise $(68 \%$ confidence interval).

decay rate of $|e\rangle$, which greatly exceeds $\Omega_{1} ; \Gamma_{e} \gg \Omega_{1}$. This means that the dressed states do not become significantly populated, and thus the levels $|e\rangle$ and $|r\rangle$ do not become significantly populated. As is shown in Fig. 7.2 the model describes the experimental data well.

The measurement of the resonance structure is repeated as $\Delta_{2}$ is varied; results are shown in Fig. 7.3. The resonance positions follow the ac-Stark formula. The results show the avoided crossing of the dressed states' eigenenergies $E_{ \pm}$. 


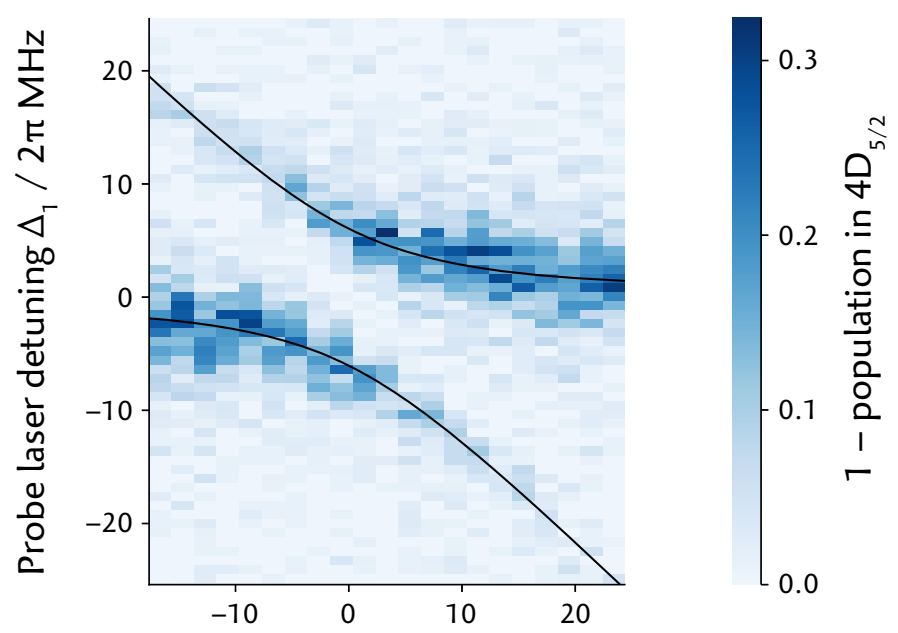

Coupling laser detuning $\Delta_{2} / 2 \pi \mathrm{MHz}$

Figure 7.3: Avoided crossing of the light-dressed states eigenenergies, described by Eq. (7.3). Resonance positions follow the ac-Stark formula $\Delta_{1}=\frac{1}{2}\left(-\Delta_{2} \pm \sqrt{{\Delta_{2}}^{2}+\Omega_{2}{ }^{2}}\right)$, which is shown by the black lines. A fit to the data returns $\Omega_{2}=2 \pi \times(12.08 \pm 0.08) \mathrm{MHz}$. Here $|r\rangle=42 S_{1 / 2}, m_{J}=-\frac{1}{2}$.

\subsection{TWO-PHOTON RABI OSCILLATIONS}

The theory proposals for systems of trapped Rydberg ions in Section 1.3.1 involve coherent excitation and deexcitation of Rydberg states. Coherent excitation and deexcitation is demonstrated in this section by two-photon Rabi oscillations. In Section $7 \cdot 4$ coherent excitation and deexcitation is carried out using stimulated Raman adiabatic passage (STIRAP). The two approaches use notably different laser parameters, for instance, synchronous UV laser pulses are used in this section, while STIRAP involves a sequence of laser pulses.

This section proceeds as follows: Experimental requirements are explained in terms of the theory behind two-photon Rabi oscillations in Section 7.3.1. Experimental results are discussed in Section 7.3.2. 


\section{7·3.1 Theoretical background}

This method results in coherent transfer of population between $|0\rangle$ and $|r\rangle$ without the lossy state $|e\rangle$ becoming populated. To avoid populating $|e\rangle$ the Rabi frequencies and detunings satisfy $\Omega_{1} \ll\left|\Delta_{1}\right|$ and $\Omega_{2} \ll\left|\Delta_{2}\right|$. Two-photon Rabi oscillations also require that the two-photon detuning between $|0\rangle$ and $|r\rangle \Delta_{2 \text {-photon }} \approx \Delta_{1}+\Delta_{2}$ is small compared with the effective two-photon Rabi frequency $\Omega_{\text {eff }}$.

Population is unlikely to be transferred to $|e\rangle$. Because of fast radiative decay out of the lossy state $|e\rangle$, with rate $\Gamma_{e}$, significant population cannot build up in $|e\rangle$. We can assume the rate of change of population in $|e\rangle$ is zero and adiabatically eliminate this state. The coupling Hamiltonian $\mathrm{H}_{\mathrm{c}}$ [Eq. (7.1)] then becomes

$$
H_{c}^{\prime}=\frac{\hbar}{2}\left(\begin{array}{cc}
-\frac{\Omega_{1}^{2}}{2 \Delta_{1}} & -\frac{\Omega_{1} \Omega_{2}}{2 \Delta_{1}} \\
-\frac{\Omega_{1} \Omega_{2}}{2 \Delta_{1}} & -\frac{\Omega_{2}^{2}}{2 \Delta_{1}}+2 \Delta_{1}+2 \Delta_{2}
\end{array}\right)
$$

in the basis $\{|0\rangle,|r\rangle\}$. The condition for two-photon resonance comes from the diagonal elements

$$
\Delta_{2 \text {-photon }}=\Delta_{1}+\Delta_{2}+\underbrace{\frac{\Omega_{1}^{2}-\Omega_{2}^{2}}{4 \Delta_{1}}}_{\text {ac-Stark shifts }}=0 .
$$

This condition already accounts for level shifts which result from the ac-Stark effect. ${ }^{1}$

The off-diagonal elements present the coupling between $|0\rangle$ and $|r\rangle$ with effective Rabi frequency

$$
\Omega_{\text {eff }}=\frac{\Omega_{1} \Omega_{2}}{2 \Delta_{1}} \text {. }
$$

Unwanted off-resonant excitation of $|e\rangle$ from $|0\rangle$ and from $|r\rangle$ occurs with rates [139]

$$
\begin{aligned}
& \mathrm{R}_{0 \rightarrow e}=\frac{\Gamma_{e}}{4} \frac{\Omega_{1}^{2}}{\Delta_{1}^{2}+\frac{\Omega_{1}^{2}}{2}+\frac{\Gamma_{e}^{2}}{4}} \approx \frac{\Gamma_{e} \Omega_{1}^{2}}{4 \Delta_{1}^{2}}, \\
& \mathrm{R}_{\mathrm{r} \rightarrow e}=\frac{\Gamma_{e}}{4} \frac{\Omega_{2}^{2}}{\Delta_{2}^{2}+\frac{\Omega_{2}^{2}}{2}+\frac{\Gamma_{e}^{2}}{4}} \approx \frac{\Gamma_{e} \Omega_{2}^{2}}{4 \Delta_{2}^{2}} .
\end{aligned}
$$

1 Interestingly these shifts cancel when $\Omega_{1}=\Omega_{2}$ for a three-level ladder system, provided that coupling to levels outside the three-level system can be ignored. 
Efficient transfer of population between $|0\rangle$ and $|r\rangle$ requires $\Omega_{\text {eff }} \gg$ $R_{0 \rightarrow e}, R_{r \rightarrow e}$. The ratios of $\Omega_{\text {eff }}$ to $R_{0 \rightarrow e}$ and to $R_{r \rightarrow e}$ can be increased by increasing the magnitudes of the detunings $\Delta_{1}$ and $\Delta_{2}$.

There is, however, a detrimental effect of increasing $\left|\Delta_{1}\right| ; \Omega_{\text {eff }}$ depends inversely on $\left|\Delta_{1}\right|$ [from Eq. (7.6)] and if $\Omega_{\text {eff }}$ is too small then Rydberg state decay $\Gamma_{\mathrm{r}}$ limits the transfer efficiency. And thus when choosing $\Delta_{1}$ and $\Delta_{2}$ a compromise must be reached between losses due to scattering off $|e\rangle$ and losses due to Rydberg state decay.

Finally laser linewidths $\Delta_{\text {laser }}$ contribute to the two-photon detuning and must be small compared with the two-photon Rabi frequency for efficient transfer between $|0\rangle$ and $|r\rangle$.

In summary, to achieve high-visibility two-photon Rabi oscillations we require

$$
\Omega_{\text {eff }} \gg \Gamma_{r}, R_{0 \rightarrow e}, R_{r \rightarrow e}, \Delta_{2-\text { photon, }} \Delta_{\text {laser }}
$$

\subsubsection{Experimental results}

Two-photon Rabi oscillations between $|0\rangle$ and $|r\rangle=46 S_{1 / 2}, m_{J}=-\frac{1}{2}$ are observed for a sideband cooled ion, but not for a Doppler cooled ion, as shown in Fig. 7.4.

We use $\Omega_{\text {eff }}=2 \pi \times(1.23 \pm 0.09) \mathrm{MHz} \gg \Gamma_{46 \mathrm{~S}}=2 \pi \times 34.4 \mathrm{kHz}$ (calculated using wavefunctions described in Section 2.2), $\mathrm{R}_{0 \rightarrow \mathrm{e}}=$ $(15.4 \pm 2.0) \mu \mathrm{s}^{-1}, \mathrm{R}_{\mathrm{r} \rightarrow \mathrm{e}}=(88 \pm 8) \mu \mathrm{s}^{-1}$ and $\Delta_{\text {laser }} \approx 2 \pi \times 100 \mathrm{kHz}$ (for each of the UV lasers - see Section 3.3.4).

The two-photon detuning $\Delta_{2 \text {-photon }}$ depends on the number of radial phonons, due to effects of the additional trapping potential experienced by the highly-polarisable Rydberg ion (Section 6.1). After radial sideband cooling, an ion has narrow radial phonon distributions and Rydberg resonances are narrow. The two-photon detuning is then $\Delta_{2 \text {-photon }}=2 \pi \times(0 \pm 150) \mathrm{kHz} \ll \Omega_{\text {eff. }}$. The criteria for twophoton Rabi oscillations [Eq. (7.8)] are satisfied and the Rabi oscillations are shown in Fig. 7.4.

The Doppler cooled ion has broader radial phonon distributions and the two-photon detuning depends on the number of radial phonons in each experimental run. The mean absolute detuning is not much smaller than the effective Rabi frequency $\left|\Delta_{2 \text {-photon }}\right| \approx 2 \pi \times$ 


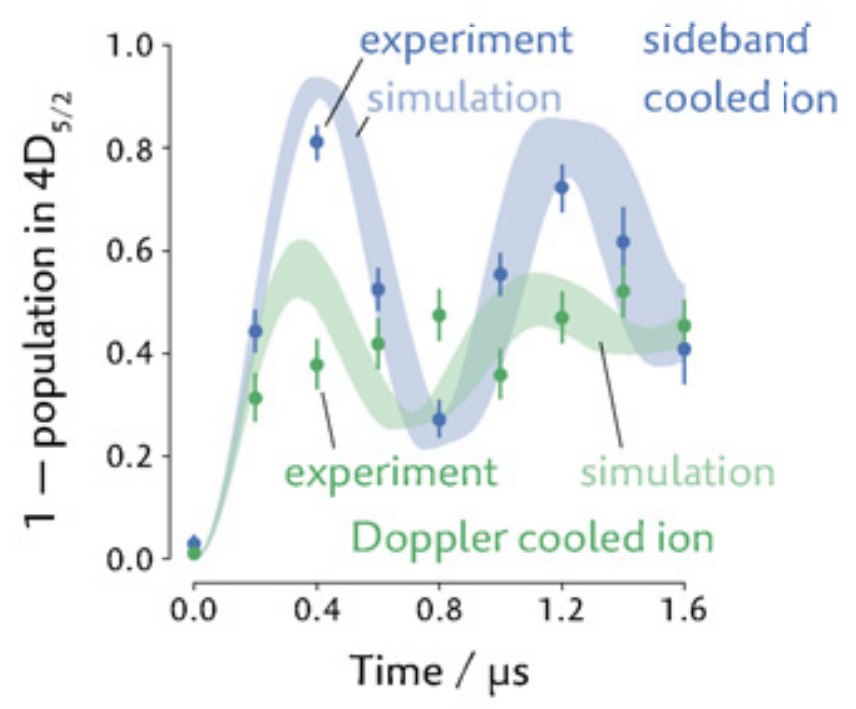

Figure 7.4: Two-photon Rabi oscillations between $|0\rangle$ and $|r\rangle$ are observed with a radial sideband cooled ion, and simulation results are consistent with experimental results. Rabi oscillations are not observed with a Doppler cooled ion, likely due to trap effects. Simulation results do not agree as well with the Doppler cooled ion experimental results. Error bars indicate quantum projection noise ( $68 \%$ confidence interval). The shaded areas shows the central $68 \%$ simulation results calculated at each time step.

$600 \mathrm{kHz} \ll \Omega_{\text {eff. }}$. This goes some way to explaining why Rabi oscillations are not observed for the Doppler cooled ion.

Some of the parameters listed thus far are based on experimentallydetermined parameters: $\Gamma_{\mathrm{e}}=2 \pi \times(4.9 \pm 0.4) \mathrm{MHz}, \Omega_{1}=2 \pi \times(23.0 \pm$ 1.5) $\mathrm{MHz}$ and $\Delta_{1}=2 \pi \times(515 \pm 15) \mathrm{MHz}$ (found using the method in Section 4.1.1), and $\Omega_{2}=2 \pi \times(55 \pm 1) \mathrm{MHz}$ (method in Section 7.2). Regarding $\Delta_{2}$ : the two-photon resonance condition [Eq. (7.5)] is met by varying the frequency of the second Rydberg excitation laser until the Rabi oscillation visibility is maximised. After radial sideband cooling the average numbers of radial phonons are estimated to be $\overline{\mathrm{n}}_{\mathrm{x}} \approx \overline{\mathrm{n}}_{\mathrm{y}} \approx 0.2$, while after Doppler cooling $\overline{\mathrm{n}}_{\mathrm{x}} \approx 10.8 \pm 3.0$ and $\bar{n}_{y} \approx 15.6 \pm 3.0$ (see Section 3.1.1). The radial trapping frequencies are altered for the Rydberg ion by $\Delta \omega_{x} \approx \Delta \omega_{y} \approx-2 \pi \times 40.5 \mathrm{kHz}$ (see Section 6.1). 


\section{Simulations of the experiment}

To find what limits the Rabi oscillation visibility, the experiment is simulated by numerically solving the Lindblad master equation using the Python framework QuTiP [140]. Experimentally-determined parameters are used.

To simulate the sideband cooled ion, the simulation includes four electronic levels $\left\{|0\rangle,|e\rangle,|r\rangle, 5 S_{1 / 2}\right\}$ and eight dimensions which represent the motional degrees of freedom in the radial directions; population is assumed to be spread over the motional degrees of freedom according to thermal distributions with an average of 0.2 phonons in each mode. The change of the trapping potential experienced by Rydberg ions is included by a phonon-number-dependent resonance shift and by phonon-number-changing transitions coupled with the Rydberg excitation. The strengths of the phonon-number-changing transitions are determined by Franck-Condon factors, and the contribution from any mismatch between the positions of the nulls of the two quadrupole trapping fields is neglected (see Section 6.1.2).

Uncertainties in the experimental parameters are accounted for using a Monte Carlo method: parameters are drawn from Gaussian probability distributions (the widths of the distributions are given by the uncertainties of the experimental parameters) and the simulation is repeated 500 times. From the set of simulations the $68 \%$ confidence interval for the $|0\rangle$ population at each simulation step is determined and plotted as the shaded area in Fig. 7.4. The simulation results match the experimental results well.

Higher visibility Rabi oscillations between low-lying states and Rydberg states have been carried out in neutral Rydberg atom experiments [52]. Further simulations indicate the oscillation visibility in our experiment is mainly limited by the Rydberg excitation lasers linewidths as well as Rydberg state decay. The visibility may be improved by exciting higher Rydberg states with longer lifetimes, by reducing the linewidths of the UV lasers and by using higher effective two-photon Rabi frequencies.

Trap effects related to the high Rydberg state polarisability (Section 6.1) are effectively mitigated by radial sideband cooling; simulations which do not account for trap effects return oscillations with 
only marginally higher visibilities $(0.1 \%$ higher $)$ than simulations which include trap effects.

The experiment with a Doppler cooled ion is also simulated using experimentally-determined parameters and a Monte Carlo method. The Doppler cooled ion has population spread over many more phonon number states than the sideband cooled ion; to speed up the Monte Carlo simulations phonon-number-changing transitions are not included. This is justified because simulations indicate that the effect of phonon-number-changing transitions on the oscillation visibility is dwarfed by the effect of the phonon-number-dependent resonance shift, provided that the positions of the nulls of the two quadrupole trapping fields overlap (Section 6.1.1).

The simulations predict low-visibility Rabi oscillations, which are not observed experimentally. It may be that the nulls of the quadrupole trapping fields are significantly separated, and that stronglydriven phonon-number-changing transitions cause dephasing which dampens the oscillations such that oscillations are not resolved (Section 6.1.2). Such phonon-number-changing transitions are stronger for high phonon number states than for low phonon number states (see Fig. 6.3). This means the phonon-number-changing transitions may be negligible for a sideband cooled ion while they may significantly decrease the oscillation visibility for a Doppler cooled ion.

\subsection{STIMULATED RAMAN ADiABATIC PASSAGE}

In addition to using synchronous UV laser pulses (as in Section 7.3), the $|0\rangle \leftrightarrow|r\rangle$ transition can be driven coherently using a sequence of pulses in a technique called stimulated Raman adiabatic passage (STIRAP).

STIRAP is a method for transferring population between two quantum states; the transfer proceeds by using light fields to couple the two states to a third intermediate state. When implemented correctly, population is transferred between the two states without the intermediate state becoming populated. And thus the transfer process does not suffer from losses due to spontaneous emission from the intermediate state, even when the STIRAP transfer occurs over timescales 
longer than the intermediate state lifetime. STIRAP has the additional advantage that it is insensitive to small variations of experimental parameters including laser powers, laser frequencies, pulse timing and pulse shapes [141].

In Section 7.4.1 the method is described further and population transfer between $|0\rangle$ and $|r\rangle$ with $(91 \pm 3) \%$ efficiency is shown. In Section 7.4.2 STIRAP is used to measure a Rydberg state lifetime. Finally in Section 7.4.3 geometric phases are introduced to a qubit state via Rydberg excitation and deexcitation by STIRAP and a single-qubit geometric gate is carried out. This gate demonstrates the basic operation of a Rydberg ion quantum computer.

\subsubsection{Coherent Rydberg excitation using STIRAP}

When the UV laser detunings cancel each other $\left(\Delta_{1}+\Delta_{2}=0\right)$ the coupling Hamiltonian [Eq. (7.1)] has a "dark" eigenstate

$$
\left|\Phi_{\text {dark }}\right\rangle=\Omega_{2} e^{i \phi}|0\rangle-\Omega_{1}|r\rangle .
$$

The dark state is named so because it does not contain any component of $|e\rangle$ and thus the Rydberg excitation laser light is not scattered by an ion in the dark state during timescales much less than the Rydberg state lifetime $\tau_{r}$.

Population transfer by STIRAP proceeds by having the state vector follow the dark state as the ratio $\frac{\Omega_{1}}{\Omega_{2}}$ is varied and the character of the dark state is changed between $|0\rangle$ and $|r\rangle$. The state vector follows the dark state provided the dark state changes adiabatically. The adiabaticity criterion [142] is

$$
|\dot{\theta}(t)| \ll \sqrt{\Omega_{1}(t)^{2}+\Omega_{2}(t)^{2}},
$$

where the mixing angle $\theta(t)$ is given by

$$
\tan \theta(t)=\frac{\Omega_{1}(t)}{\Omega_{2}(t)}
$$




\section{STIRAP pulse sequence}

We implement STIRAP by varying $\Omega_{1}$ and $\Omega_{2}$ sinusoidally during the rise time $t_{\text {rise. }}$ While $0 \leqslant t \leqslant t_{\text {rise }}$

$$
\begin{aligned}
& \Omega_{1}(t)=\Omega_{\text {peak }} \sin \frac{\pi t}{2 t_{\text {rise }}}, \\
& \Omega_{2}(t)=\Omega_{\text {peak }} \cos \frac{\pi t}{2 t_{\text {rise }}},
\end{aligned}
$$

where the peak Rabi frequencies $\Omega_{\text {peak }}$ are the same for both $\Omega_{1}$ and $\Omega_{2}$. Pulse shaping is achieved by driving acousto-optic modulators (AOMs) using arbitrary waveform generators (Section 3.3.2). The shapes and the temporal overlap of the pulses are checked using photodiodes. Pulse sequences are shown in Fig. 7.5.

The order of the pulses is counter-intuitive; to transfer population from $|0\rangle \rightarrow|r\rangle$ the $306 \mathrm{~nm}$ laser pulse is applied before the $243 \mathrm{~nm}$ laser pulse. Because the pulses are sinusoidally-shaped the mixing angle [Eq. (7.11)] changes at a constant rate during the rise time $t_{\text {rise }}$

$$
\theta(t)=\frac{\pi t}{2 t_{\text {rise }}}
$$

and the adiabaticity criterion takes the simple form

$$
\frac{\pi}{2 t_{\text {rise }}} \ll \Omega_{\text {peak }} .
$$

In the experiment the Rabi frequencies $\Omega_{1}, \Omega_{2}$ and the detunings $\Delta_{1}, \Delta_{2}$ are found using the methods in Sections 4.1.1 and 7.2. The requirement $\Delta_{1}+\Delta_{2}=0$ is met by setting $\Delta_{1}=\Delta_{2}=0$. $\Omega_{\text {peak }}$ is limited by the maximum value of $\Omega_{1}$ and thus by the $243 \mathrm{~nm}$ laser light intensity at the position of the ion. We use $t_{\text {rise }}=200 \mathrm{~ns}$ and $\Omega_{\text {peak }} \approx 2 \pi \times 47 \mathrm{MHz}$ and satisfy the adiabaticity criterion $\frac{\pi}{2 \mathrm{t}_{\text {rise }}}=$ $2 \pi \times 1.25 \mathrm{MHz} \ll \Omega_{\text {peak }}=2 \pi \times 47 \mathrm{MHz}$. $t_{\text {rise }}$ must be long for the state vector to adiabatically follow the dark state, however if it is too long there are losses from Rydberg state decay $\left(\tau_{r} \approx 2.3 \mu\right.$ s for $\left.42 S_{1 / 2}\right)$ and from decoherence due to finite laser linewidths. $t_{\text {rise }}=200 \mathrm{~ns}$ is chosen as a compromise. The Rydberg excitation lasers linewidths are each estimated to be $\sim 2 \pi \times 100 \mathrm{kHz}$ (Section 3.3.4). 
a single STIRAP pulse sequence

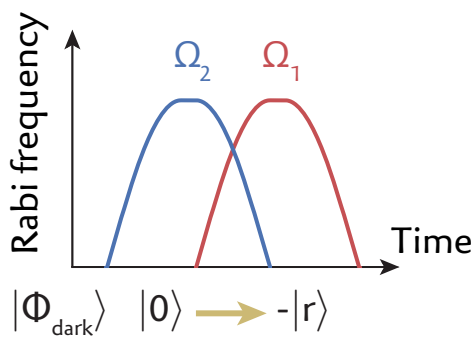

b

double STIRAP pulse sequence

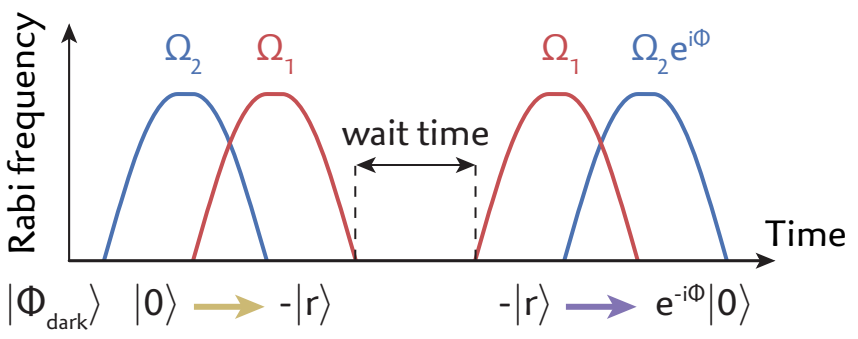

C shortened double STIRAP pulse sequence

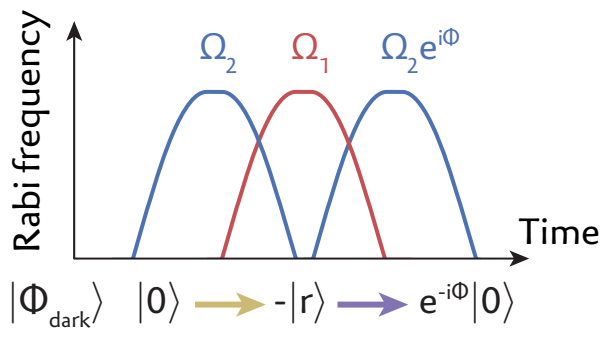

Figure 7.5: STIRAP pulse sequences. The evolution of the dark state is written beneath each pulse sequence. The third sequence is a condensed version of the second sequence.

Detection of population transfer by STIRAP

State detection in our system takes longer than the lifetime of $|r\rangle$. As is described in Sections 4.1.1 and 4.3, detection of population excited from $|0\rangle$ to $|e\rangle$ or from $|0\rangle$ to $|r\rangle$ relies on decay from $|e\rangle$ or $|r\rangle$ to $5 S_{1 / 2}$ and $4 \mathrm{D}_{3 / 2}$; population in $5 \mathrm{~S}_{1 / 2}$ and $4 \mathrm{D}_{3 / 2}$ is then distinguished from population in $|0\rangle$ by electron shelving (Section 3.1.4). This method does not distinguish successful transfer to $|r\rangle$ from scattering off $|e\rangle$; 
in both cases there is decay to $5 S_{1 / 2}$ and $4 D_{3 / 2}$ and thus this method is not able to confirm population transfer by STIRAP.

Instead population transfer is detected by comparing the population residing in $|0\rangle$ after application of the single STIRAP pulse sequence and application of the double STIRAP pulse sequence (pulse sequences are shown in Fig. 7.5). After the single STIRAP pulse sequence $\left(95_{-5}^{+2}\right) \%$ of the population is removed from $|0\rangle$. After the double STIRAP pulse sequence up to $58 \%$ of the population resides in state $|0\rangle$. This indicates the second set of pulses in the double STIRAP pulse sequence returns population to $|0\rangle$ and thus demonstrates excitation and deexcitation by STIRAP. These results are shown in Fig. 7.6. The return of population to $|0\rangle$ is not perfect because the state vector

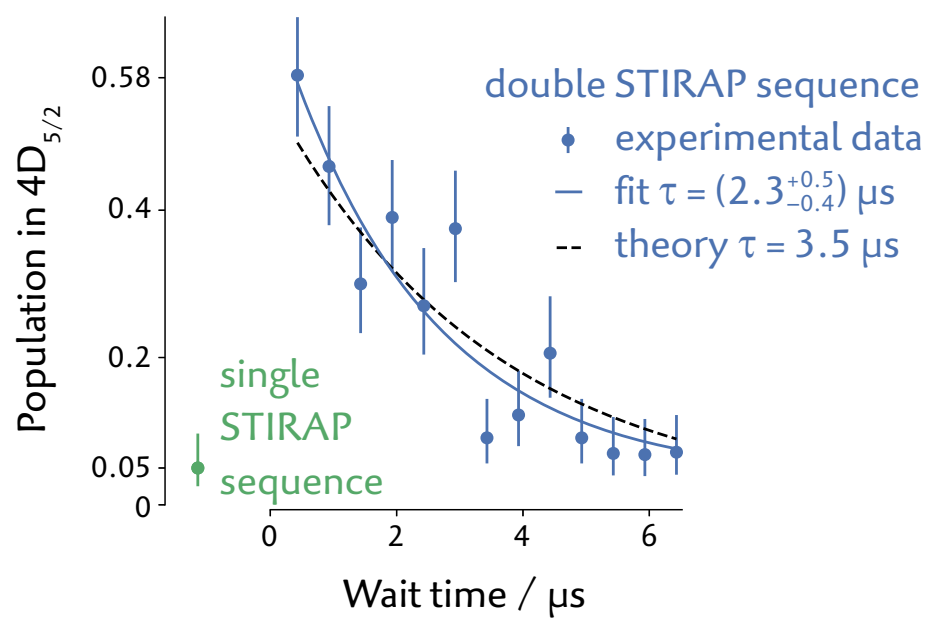

Figure 7.6: Coherent Rydberg excitation by STIRAP shown by comparing the results of applying the single and the double STIRAP pulse sequences to an ion initially in state $|0\rangle$. $\left(5_{-3}^{+5}\right) \%$ of the population remains in $|0\rangle$ after the single STIRAP pulse sequence; the rest of the population is transferred to $5 S_{1 / 2}$ via $|e\rangle$ or $|r\rangle$. Most of the population may lie in $|0\rangle$ after the double STIRAP pulse sequence, this shows population is successfully excited to the Rydberg state $|r\rangle=42 S_{1 / 2}, m_{J}=-\frac{1}{2}$ then de-excited to $|0\rangle$. The $42 S_{1 / 2}$ state lifetime is obtained by varying the wait time between the two sets of STIRAP pulses and measuring the returned population. Error bars indicate quantum projection noise $(68 \%$ confidence interval). 
does not follow the dark state perfectly, due to Rydberg state decay, finite laser linewidths and the short $t_{\text {rise }}$.

Using the shortened double STIRAP pulse sequence in Fig. 7.5(c) the detrimental effect of Rydberg state decay is reduced and $\left(83_{-6}^{+5}\right) \%$ of the population is returned to $|0\rangle$. This indicates a transfer efficiency $\sqrt{\left(83_{-6}^{+5}\right) \%}=(91 \pm 3) \%$, which exceeds the highest STIRAP efficiencies reported in systems of neutral Rydberg atoms (60\%) [143-145]. In Section 7.4.3 ways to improve the transfer efficiency are discussed.

\subsubsection{Measurement of a Rydberg state lifetime}

In this section the first lifetime measurement of a trapped Rydberg ion is described. This measurement relies upon coherent Rydberg excitation and deexcitation using STIRAP.

During the wait time between the two sets of pulses in the double STIRAP pulse sequence [see Fig. 7.5(b)] population may decay from $|r\rangle$; this population is not returned to $|0\rangle$ by the second set of STIRAP pulses. We determine the lifetime of $|r\rangle$ by measuring the population returned to $|0\rangle$ as the wait time is varied. We measure $\tau_{\exp }=$ $\left(2.3_{-0.4}^{+0.5}\right) \mu \mathrm{s}$ for $42 \mathrm{~S}_{1 / 2}$, as shown in Fig. 7.6.

The theoretical value for the lifetime of $42 \mathrm{~S}_{1 / 2}$ is $\tau_{300 \mathrm{~K}}=3.5 \mu \mathrm{s}$ when the surroundings have temperature $300 \mathrm{~K}$ (similar to the laboratory temperature). This value accounts for radiative decay as well as transitions driven by blackbody radiation (see Section 2.4).

The discrepancy between the theory value and the experimental value of the lifetime may be due to imperfect extinction of $306 \mathrm{~nm}$ laser light during the wait time of the double STIRAP pulse sequence. During this time weak $306 \mathrm{~nm}$ laser light could drive the $|\mathrm{r}\rangle \leftrightarrow|e\rangle$ transition and population may be lost due to decay from $|e\rangle$. As a result the measured lifetime of $42 \mathrm{~S}_{1 / 2}$ would be shortened.

Comparison between the theoretical value and the experimental value of the lifetime suggests there is no significant lifetime reduction because of confinement in the trap. This is an important result; if the Rydberg state lifetime was significantly shortened by the trap the viability of Rydberg ions as a quantum technology may be lim- 
ited, since Rydberg state lifetimes place fundamental limits on gate fidelities [8o] and resonance linewidths.

\subsubsection{Geometric phase measurements}

A geometric phase is introduced to qubit state $|0\rangle$ via Rydberg excitation and deexcitation using a STIRAP pulse sequence. This section proceeds as follows: the theory behind geometric phases is established, the experimental implementation is then described, the measurement of the geometric phase using a Ramsey-type experiment is presented, and finally a single-qubit Rydberg gate which uses the geometric phase is discussed.

\section{Theory behind geometric phases}

When a quantum system is changed slowly in a cyclic fashion the eigenstates of the Hamiltonian accumulate dynamic and geometric phases [146]. The dynamic phase $\phi_{\text {dyn }}$ results from the evolution of an eigenenergy; the eigenstate $|n(t)\rangle$ with eigenenergy $\epsilon_{n}(t)$ which evolves during a cycle with period $T$ accumulates dynamic phase

$$
\phi_{\text {dyn }}=-\frac{1}{\hbar} \int_{0}^{T} \epsilon_{n}\left(t^{\prime}\right) d t^{\prime} .
$$

The geometric phase accumulated is given by

$$
\begin{aligned}
\gamma & =i \int_{0}^{T}\left\langle n\left(\vec{R}\left(t^{\prime}\right)\right)\left|\frac{d}{d t^{\prime}}\right| n\left(\vec{R}\left(t^{\prime}\right)\right)\right\rangle d t^{\prime} \\
& =i \oint_{\mathcal{C}}\left\langle n\left(\vec{R}\left(t^{\prime}\right)\right) \mid \vec{\nabla}_{\vec{R}} n\left(\vec{R}\left(t^{\prime}\right)\right)\right\rangle \cdot d \vec{R},
\end{aligned}
$$

where the quantum system is parameterised by a set of parameters $\vec{R}$ and the system follows the closed path $\mathcal{C}$ in the parameter space such that $\vec{R}(T)=\vec{R}(0)$. Unlike the dynamic phase, the geometric phase does not depend on the rate at which the system evolves along $\mathrm{C}$.

Introduction of a geometric phase in the experiment

In our system we accumulate a geometric phase during the shortened double STIRAP pulse sequence by varying the mixing angle $\theta$ [see 
Eq. (7.11)] and the phase difference between the UV laser fields within the rotating frame $\phi$ in a closed cycle, following a protocol recently used with a solid-state qubit [147]. The cycle is parameterised by $\vec{R}=\left(\begin{array}{l}\theta \\ \phi\end{array}\right)$ and follows

$$
\left(\begin{array}{l}
0 \\
0
\end{array}\right) \rightarrow\left(\begin{array}{l}
\pi \\
0
\end{array}\right) \rightarrow\left(\begin{array}{l}
\pi \\
\Phi
\end{array}\right) \rightarrow\left(\begin{array}{l}
0 \\
\Phi
\end{array}\right) \rightarrow\left(\begin{array}{l}
0 \\
0
\end{array}\right) .
$$

During the cycle the dark state traverses the surface of the Bloch sphere spanned by $|0\rangle$ and $|r\rangle$ in Fig. 7.7(a); the mixing angle $\theta$ corresponds to the polar angle, the phase difference $\phi$ corresponds to the azimuthal angle.

The dark state moves from the 0-pole to the r-pole, then back to the 0-pole. The phase of the second Rydberg excitation laser light is changed by $\Phi$ when the dark state is at the $r$-pole, that is when $\Omega_{2}=0$, and the dark state returns to the 0-pole along a different meridian (line of constant $\phi$ ). The complete path takes the form of a tangerine slice with wedge angle $\Phi .^{2}$ The path circumscribes solid angle $2 \Phi$ and from Eqs. $(7.9,7.17)$ the geometric phase $-\Phi$ is accumulated throughout the process [147]. During the cycle the dark state changes from $|0\rangle \rightarrow-|r\rangle \rightarrow e^{-i \Phi}|0\rangle$.

\section{Measurement of the geometric phase}

While the global phase of a quantum state cannot be measured, phase differences between quantum states can be measured. The geometric phase introduced to qubit state $|0\rangle$ during the cycle is measured via a Ramsey experiment (which uses the qubit state $|1\rangle \equiv 5 S_{1 / 2}, m_{J}=-\frac{1}{2}$ as a phase reference) as follows:

1. The ion is prepared in superposition state $|\psi\rangle=|0\rangle+|1\rangle$ as described in Section 3.1.3.

2 Note that $\phi$ represents the variable phase difference between the two UV laser fields within the rotating frame, while $\Phi$ represents the phase step introduced to the $306 \mathrm{~nm}$ laser light during the sequence and thus the wedge angle of the path followed by the dark state in Fig. $7 \cdot 7(\mathrm{a})$. 


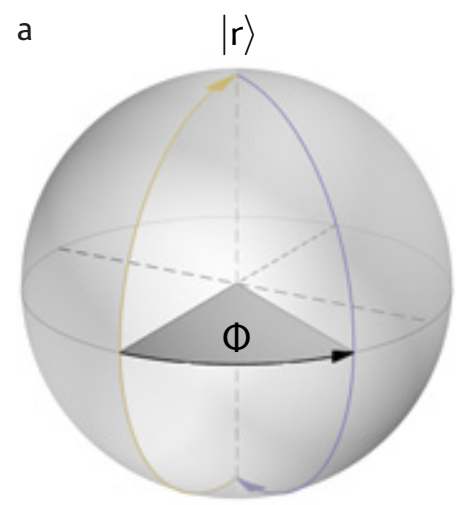

$|0\rangle$

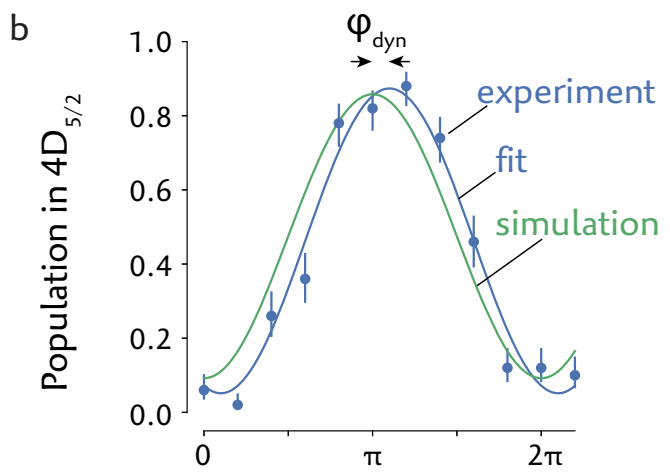

Phase difference between $306 \mathrm{~nm}$ laser pulses $\Phi$

Figure 7.7: Accumulation of a geometric phase during a Rydberg excitation process. (a) During the shortened double STIRAP pulse sequence the dark state moves in a 'tangerine-slice' trajectory on the surface of the Bloch sphere spanned by $|0\rangle$ and $|r\rangle$. The wedge angle is determined by the phase step of the $306 \mathrm{~nm}$ laser light. The mixing angle in Eq. (7.11) corresponds to the polar angle on the Bloch sphere. A geometric phase results from the curvature of the area enclosed by the trajectory. (b) The geometric phase is measured by using the qubit state $|1\rangle$ as a phase reference, and measuring the phase difference between $|0\rangle$ and $|1\rangle$ by a $\hat{\sigma}_{y^{-}}$ measurement. Here $|r\rangle=42 S_{1 / 2}, m_{J}=-\frac{1}{2}$. Error bars indicate quantum projection noise ( $68 \%$ confidence interval).

2. The shortened double STIRAP pulse sequence is carried out and the state evolves to $|\psi\rangle=e^{-i \Phi}|0\rangle+|1\rangle$ (with perfect transfer efficiency).

3. A measurement in the $\hat{\sigma}_{x}$-basis carried out: the state is rotated about the $\hat{y}$ axis by $\frac{\pi}{2}$ to become $|\psi\rangle=-\sin \frac{\Phi}{2}|0\rangle+\cos \frac{\Phi}{2}|1\rangle$ and then electron shelving is used to distinguish population in the $\{|0\rangle,|1\rangle\}$ basis (Section 3.1.4). The fraction of the population projected onto $|0\rangle$ is $\sin ^{2} \frac{\Phi}{2}$.

This Ramsey experiment is repeated as the laser phase step $\Phi$ is varied, the results are shown in Fig. $7 \cdot 7(\mathrm{~b})$. Oscillatory behaviour is observed, confirming that a geometric phase is acquired during the 
shortened double STIRAP pulse sequence. The contrast of the oscillation $\mathcal{C}=(82 \pm 4) \%$ is less than unity because of the imperfect STIRAP transfer efficiency and decoherence due to finite laser linewidths. The average of the oscillation is less than 0.5 because population decays from $|r\rangle$ outside the $\{|0\rangle,|1\rangle\}$ manifold (mostly to $5 S_{1 / 2}, m_{J}=+\frac{1}{2}$ ).

This experiment is simulated by numerically solving the Lindblad master equation for the five-level system $\left\{|0\rangle,|e\rangle,|r\rangle,|1\rangle, 5 S_{1 / 2}, m_{J}=\right.$ $\left.+\frac{1}{2}\right\}$ using experimentally-determined parameters with the Python framework QuTiP [140]. Excellent agreement is observed between the experimental and simulation results. Further simulations indicate the dynamic phase offset in the experimental data $\phi_{\text {dyn }}=(18 \pm 4)^{\circ}$ may be accounted for by a small two-photon detuning $(2 \pi \times 100 \mathrm{kHz})$ from the $|0\rangle \leftrightarrow|r\rangle$ resonance and by the light shift from the second Rydberg excitation laser field on $|1\rangle$.

The simulation results show a lower contrast than the experimental results. Using the experimentally-determined parameters, simulations predict STIRAP transfer efficiency $90 \%$. Repeating the simulations with Rydberg excitation laser linewidths $2 \pi \times 64 \mathrm{kHz}$ the simulation contrast matches the experimental contrast and the simulations predict STIRAP transfer efficiency $91 \%$. The good agreement between simulation results and experimental results indicate the simulations model our system well.

Simulations indicate the STIRAP transfer efficiency is limited by Rydberg state decay, Rydberg excitation laser linewidths and imperfect adiabatic following during the rise time $t_{\text {rise }}$. The transfer efficiency may be improved by using higher Rydberg-excitation laser light intensities, by exciting higher Rydberg states with longer lifetimes and by improving the frequency stabilisation of the Rydberg-excitation lasers.

\section{Single-qubit Rydberg gate}

The geometric phase accumulated during a cycle is insensitive to small changes in the path followed and thus geometric manipulation of quantum systems may allow noise-resilient quantum computation $[148,149]$. 
We use the shortened STIRAP pulse sequence [Fig. 7.5(c)] to implement a single-qubit geometric phase gate. This gate is characterised using quantum process tomography for the case with phase step $\Phi=\pi$. Descriptions of quantum state tomography and quantum process tomography are available in [150, 151], brief descriptions of the techniques follow.

Quantum state tomography allows an unknown quantum state to be reconstructed. The same unknown state is prepared multiple times and measured using different measurement operators. The measurement operators must form a complete basis set in the Hilbert space of the system. In our system we measure a single qubit and use the Pauli operators $\left\{\hat{\sigma}_{x}, \hat{\sigma}_{y}, \hat{\sigma}_{z}\right\}$ as measurement operators, as described in Section 3.1.4. Measurements with the same measurement operator are typically repeated $\approx 50$ times. Each measurement produces a binary outcome; measurements must be repeated for quantum states to be determined accurately.

With quantum process tomography a process is characterised by carrying out the process on known states then carrying out quantum state tomography on the post-process states. For a system with a d-dimensional Hilbert space copies of $\mathrm{d}^{2}$ input states are required. Input states are chosen such that their density matrices form a basis set for the space of matrices. For our single-qubit system $d=2$ and thus we prepare copies of the four input states $|0\rangle,|0\rangle+|1\rangle,|0\rangle+i|1\rangle$ and $|1\rangle$. State preparation is described in Section 3.1.3.

A process is conventionally described by a process matrix or $\chi^{-}$ matrix. We use a maximum likelihood estimation in determining the $\chi$-matrix. The reconstructed $\chi$-matrix is shown in Fig. $7.8(\mathrm{a})$. The ideal process is equivalent to a $\hat{\sigma}_{z}$ rotation. The process fidelity of $(78 \pm 4) \%$ is the overlap of the reconstructed $x$-matrix with the $x$ matrix for a $\hat{\sigma}_{z}$ rotation. The errors are estimated by a Monte Carlo method; due to quantum projection noise the measurement results have uncertainties described by binomial probability distributions. Samples are repeatedly drawn from the probability distributions and $\chi$ is repeatedly reconstructed. The process fidelities of the different $\chi$-matrices are found and their distribution is used to estimate the uncertainty in the estimate of the process fidelity. 

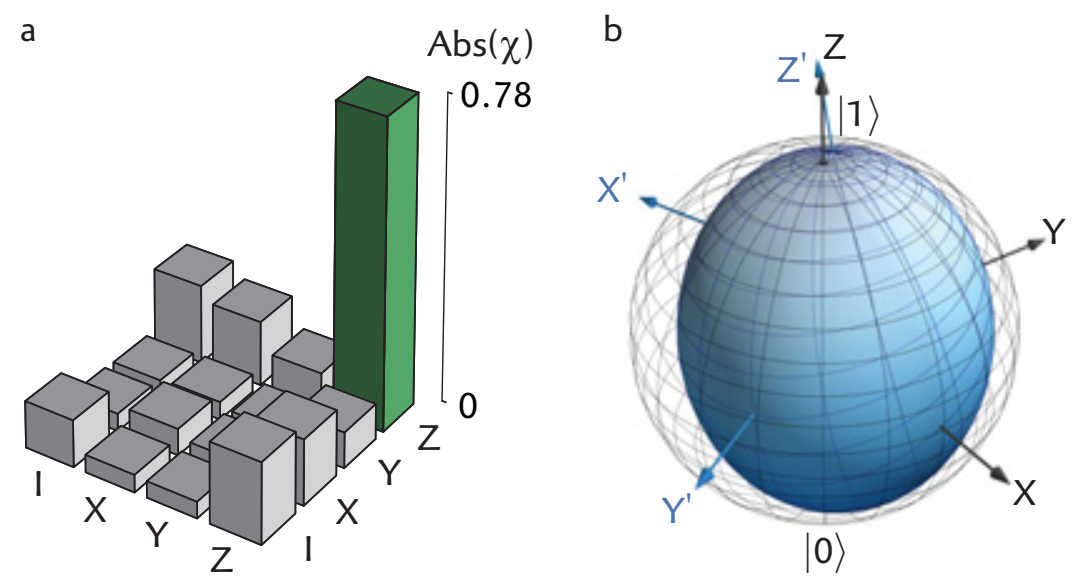

Figure 7.8: Process tomography of the shortened double STIRAP pulse sequence using $\Phi=\pi$. (a) The absolute value of the reconstructed process matrix is shown. In the ideal process the ZZ-bar height is 1 , the measured process fidelity is $(78 \pm 4) \%$. (b) The process matrix is visualised by comparing the pre-process (meshed) and post-process (blue) Bloch spheres: the Bloch sphere is rotated about the $\mathrm{z}$-axis by $\approx \pi$ and inefficiencies cause the sphere to shrink. Here $|r\rangle=42 S_{1 / 2}, m_{J}=-\frac{1}{2}$.

The reconstructed $\chi$-matrix may be used to predict the effect of the process on any qubit state. This is represented in Fig. 7.8(b); initial qubit states sitting on the meshed Bloch sphere are transformed by the process and the output states sit on the solid Bloch sphere. In the ideal process the solid Bloch sphere would be rotated about the z-axis by $\pi$; the imperfect rotation in Fig. $7.8(\mathrm{~b})$ is due to the dynamic phase $\phi_{\text {dyn }}$. The imperfect STIRAP efficiency causes shrinking of the 0-pole of the Bloch sphere. The asymmetry about the z-axis is likely caused by imperfect Ramsey pulses.

This single-qubit phase gate is not the most efficient way to introduce a $\pi$-phase to a single qubit; this is rather a demonstration of a quantum gate involving a trapped Rydberg ion. This single-qubit gate may be extended to a two-qubit phase gate in which phases are generated by strong interaction between Rydberg ions. Such a gate - which involves excitation of Rydberg states via STIRAP — was proposed in [152, 153]. This gate requires strong interaction between 
Rydberg ions. MW-dressed Rydberg states can have large dipole moments [49, 68]. Strong dipole-dipole interaction between such MWdressed Rydberg states have recently been measured in our laboratory. We are currently trying to implement the two-qubit phase gate. 
The theory investigation by Müller et al. [49] predicted that trapping of Rydberg ions in a linear Paul trap is feasible and that strong interactions between trapped Rydberg ions may be used for carrying out fast quantum gates. Trapped Rydberg ions are thus an interesting platform for quantum information processing, and various other proposals are summarised in Section 1.3.1. These proposals have motivated two experimental investigations of trapped Rydberg ions: ${ }^{40} \mathrm{Ca}^{+}$ ions are excited to Rydberg states using a single-photon excitation scheme in Mainz, while ${ }^{88} \mathrm{Sr}^{+}$ions are excited to Rydberg states by two-photon transitions in the experiment described in this dissertation.

The two-photon excitation scheme makes it easier to work within the Lamb-Dicke regime, as discussed in Chapter 4. This allows us to attain narrower Rydberg resonance lines than the Mainz experiment. With narrow resonance lines we have investigated effects of the strong trapping electric fields on the sensitive Rydberg ions, which are presented in Chapter 6. After trap effects were mitigated, Rydberg states were coherently excited and a single-qubit phase gate was carried out, as described in Chapter 7 .

The next step towards using trapped Rydberg ions for quantum information processing is to carry out two-qubit gates which take advantage of strong Rydberg interactions. This is the PhD thesis work of Fabian Pokorny. Towards this goal Fabian and Chi Zhang have

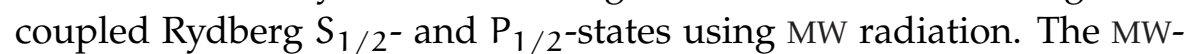
dressed states have dipole moments and ions in these states interact strongly with each other by dipole-dipole interaction. When Rabi oscillations are driven between the low-lying state $|0\rangle$ and a MW-dressed Rydberg state Fabian and Chi have observed a partial Rydberg blockade - this is a signature of interacting Rydberg ions.

Different two-qubit gates may be implemented using strong Rydberg interactions. A Rydberg blockade gate can be carried out if 
the Rydberg-excitation Rabi frequency is much lower than the interaction strength, as was done with neutral Rydberg atoms [54]. The phase gate demonstrated in Section 7.4.3 may be extended to a twoqubit controlled phase gate [152, 153] if a Rydberg-excitation Rabi frequency much higher than the interaction strength is used.

After typically several hundred excitations to Rydberg states ions are lost by double ionisation. Until recently these events interrupted the experiment by around 20 minutes and made it cumbersome to collect data. Ablation loading of ${ }^{88} \mathrm{Sr}^{+}$ions has been introduced to the experiment by Andreas Pöschl and Quentin Bodart, and the ion loading time is reduced; double ionisation events now interrupt the experiment by less than 30 seconds. Future Rydberg ion experiments may find it invaluable to shuttle trapped ions between a loading zone containing an ion reservoir and an experiment zone, as was envisaged in a Mainz design [75]. 
1. Itano, W. M. Perspectives on the quantum Zeno paradox. J. Phys. Conf. Ser. 196, 012018 (2009).

2. Kocsis, S. et al. Observing the Average Trajectories of Single Photons in a Two-Slit Interferometer. Science 332, 1170-1173 (2011).

3. Lapkiewicz, R. et al. Experimental non-classicality of an indivisible quantum system. Nature 474, 490 (2011).

4. Ringbauer, M. et al. Measurements on the reality of the wavefunction. Nat. Phys. 11, 249 (2015).

5. Nigg, D. et al. Can different quantum state vectors correspond to the same physical state? An experimental test. New J. Phys. 18, 013007 (2016).

6. Shalm, L. K. et al. Strong Loophole-Free Test of Local Realism. Phys. Rev. Lett. 115, 250402 (2015).

7. Giustina, M. et al. Significant-Loophole-Free Test of Bell's Theorem with Entangled Photons. Phys. Rev. Lett. 115, 250401 (2015).

8. Hensen, B. et al. Loophole-free Bell inequality violation using electron spins separated by 1.3 kilometres. Nature 526, 682 (2015).

9. Quantum Technologies Roadmap http : / / qurope. eu / system / files / QT\%20Roadmap \%202016_0 . pdf. Accessed: 29/o1/2018. (2016).

10. Ladd, T. D. et al. Quantum computers. Nature 464, 45 (2010).

11. Monz, T. et al. Realization of a scalable Shor algorithm. Science 351, 1068-1070 (2016).

12. Blatt, R. \& Roos, C. F. Quantum simulations with trapped ions. Nat. Phys. 8, 277 (2012). 
13. Duan, L.-M. \& Monroe, C. Colloquium: Quantum networks with trapped ions. Rev. Mod. Phys. 82, 1209-1224 (2010).

14. Facon, A. et al. A sensitive electrometer based on a Rydberg atom in a Schrödinger-cat state. Nature 535, 262-265 (2016).

15. Wang, Y. et al. Single-qubit quantum memory exceeding tenminute coherence time. Nat. Photonics 11, 646-650 (2017).

16. F. G. Major, V. N. G. \& Werth, G. Charged Particle Traps (SpringerVerlag Berlin Heidelberg, 2005).

17. Brownnutt, M., Kumph, M., Rabl, P. \& Blatt, R. Ion-trap measurements of electric-field noise near surfaces. Rev. Mod. Phys. 87, 1419-1482 (2015).

18. Günther Werth, V. N. G. \& Major, F. G. Charged Particle Traps II: Applications (Springer-Verlag Berlin Heidelberg, 2009).

19. Harty, T. P. et al. High-Fidelity Preparation, Gates, Memory, and Readout of a Trapped-Ion Quantum Bit. Phys. Rev. Lett. 113, 220501 (2014).

20. Ballance, C. J., Harty, T. P., Linke, N. M., Sepiol, M. A. \& Lucas, D. M. High-Fidelity Quantum Logic Gates Using Trapped-Ion Hyperfine Qubits. Phys. Rev. Lett. 117, 060504 (2016).

21. Gaebler, J. P. et al. High-Fidelity Universal Gate Set for ${ }^{9} \mathrm{Be}^{+}$ Ion Qubits. Phys. Rev. Lett. 117, 060505 (2016).

22. Wang, X.-L. et al. 18-qubit entanglement with photon's three degrees of freedom. arXiv: 1801.04043 (2018).

23. Martinez, E. A. et al. Real-time dynamics of lattice gauge theories with a few-qubit quantum computer. Nature 534, 516 (2016).

24. Zhang, J. et al. Observation of a discrete time crystal. Nature 543, 217 (2017).

25. Smith, J. et al. Many-body localization in a quantum simulator with programmable random disorder. Nat. Phys. 12, 907 (2016).

26. Zhang, J. et al. Observation of a many-body dynamical phase transition with a 53-qubit quantum simulator. Nature 551, 601 (2017). 
27. Barreiro, J. T. et al. An open-system quantum simulator with trapped ions. Nature 470, 486 (2011).

28. Clos, G., Porras, D., Warring, U. \& Schaetz, T. Time-Resolved Observation of Thermalization in an Isolated Quantum System. Phys. Rev. Lett. 117, 170401 (2016).

29. Neyenhuis, B. et al. Observation of prethermalization in longrange interacting spin chains. Sci. Adv. 3 (2017).

30. Eisert, J., Friesdorf, M. \& Gogolin, C. Quantum many-body systems out of equilibrium. Nat. Phys. 11, 124 (2015).

31. Roßnagel, J. et al. A single-atom heat engine. Science 352, 325329 (2016).

32. O'Malley, P. J. J. et al. Scalable Quantum Simulation of Molecular Energies. Phys. Rev. X 6, 031007 (2016).

33. Shen, Y. et al. Quantum implementation of the unitary coupled cluster for simulating molecular electronic structure. Phys. Rev. A 95, 020501 (2017).

34. Vandersypen, L. M. K. et al. Experimental realization of Shor's quantum factoring algorithm using nuclear magnetic resonance. Nature 414, 883 (2001).

35. Lu, C.-Y., Browne, D. E., Yang, T. \& Pan, J.-W. Demonstration of a Compiled Version of Shor's Quantum Factoring Algorithm Using Photonic Qubits. Phys. Rev. Lett. 99, 250504 (2007).

36. Lanyon, B. P. et al. Experimental Demonstration of a Compiled Version of Shor's Algorithm with Quantum Entanglement. Phys. Rev. Lett. 99, 250505 (2007).

37. Lucero, E. et al. Computing prime factors with a Josephson phase qubit quantum processor. Nat. Phys. 8, 719 (2012).

38. Chiaverini, J. et al. Realization of quantum error correction. Nature 432, 602 (2004).

39. Schindler, P. et al. Experimental Repetitive Quantum Error Correction. Science 332, 1059-1061 (2011).

40. Nigg, D. et al. Quantum computations on a topologically encoded qubit. Science 345, 302-305 (2014). 
41. Leibfried, D. et al. Toward Heisenberg-Limited Spectroscopy with Multiparticle Entangled States. Science 304, 1476-1478 (2004).

42. Roos, C. F., Chwalla, M., Kim, K., Riebe, M. \& Blatt, R. 'Designer atoms' for quantum metrology. Nature 443, 316 (2006).

43. Chou, C. W., Hume, D. B., Koelemeij, J. C. J., Wineland, D. J. \& Rosenband, T. Frequency Comparison of Two High-Accuracy $\mathrm{Al}^{+}$Optical Clocks. Phys. Rev. Lett. 104, 070802 (2010).

44. Huntemann, N., Sanner, C., Lipphardt, B., Tamm, C. \& Peik, E. Single-Ion Atomic Clock with $3 \times 10^{-18}$ Systematic Uncertainty. Phys. Rev. Lett. 116, 063001 (2016).

45. Mølmer, K. \& Sørensen, A. Multiparticle Entanglement of Hot Trapped Ions. Phys. Rev. Lett. 82, 1835-1838 (1999).

46. Wineland, D. J. et al. Experimental Issues in Coherent QuantumState Manipulation of Trapped Atomic Ions. J. Res. Natl. Inst. Stand. Technol. 103, 259-328 (1998).

47. Korenblit, $S$ et al. Quantum simulation of spin models on an arbitrary lattice with trapped ions. New J. Phys. 14, 095024 (2012).

48. Kielpinski, D., Monroe, C. \& Wineland, D. J. Architecture for a large-scale ion-trap quantum computer. Nature 417, 709 (2002).

49. Müller, M., Liang, L., Lesanovsky, I. \& Zoller, P. Trapped Rydberg ions: from spin chains to fast quantum gates. New J. Phys. 10, 093009 (2008).

50. Lebedev, V. \& Beigman, I. Physics of Highly Excited Atoms and Ions (Springer, Berlin, 1998).

51. Hans A. Bethe, E. E. S. Quantum Mechanics of One- and TwoElectron Atoms (Springer, Boston, MA, 1977).

52. Browaeys, A., Barredo, D. \& Lahaye, T. Experimental investigations of dipole-dipole interactions between a few Rydberg atoms. J. Phys. B 49, 152001 (2016).

53. Hagley, E. et al. Generation of Einstein-Podolsky-Rosen Pairs of Atoms. Phys. Rev. Lett. 79, 1-5 (1997). 
54. Isenhower, L. et al. Demonstration of a Neutral Atom Controlled-NOT Quantum Gate. Phys. Rev. Lett. 104, 010503 (2010).

55. Gross, C. \& Bloch, I. Quantum simulations with ultracold atoms in optical lattices. Science 357, 995-1001 (2017).

56. Saffman, M. Quantum computing with atomic qubits and Rydberg interactions: progress and challenges. J. Phys. B 49, 202001 (2016).

57. Firstenberg, O, Adams, C. S. \& Hofferberth, S. Nonlinear quantum optics mediated by Rydberg interactions. J. Phys. B 49, 152003 (2016).

58. Miller, $\mathrm{R}$ et al. Trapped atoms in cavity QED: coupling quantized light and matter. J. Phys. B 38, S551 (2005).

59. Raimond, J. M., Brune, M. \& Haroche, S. Manipulating quantum entanglement with atoms and photons in a cavity. Rev. Mod. Phys. 73, 565-582 (2001).

6o. Bendkowsky, V. et al. Observation of ultralong-range Rydberg molecules. Nature 458, 1005 (2009).

61. Niederprüm, T. et al. Observation of pendular butterfly Rydberg molecules. Nat. Commun. 7, 12820 (2016).

62. Kübler, H., Shaffer, J. P., Baluktsian, T., Löw, R. \& Pfau, T. Coherent excitation of Rydberg atoms in micrometre-sized atomic vapour cells. Nat. Photonics 4, 112 (2010).

63. Tauschinsky, A., Thijssen, R. M. T., Whitlock, S., van Linden van den Heuvell, H. B. \& Spreeuw, R. J. C. Spatially resolved excitation of Rydberg atoms and surface effects on an atom chip. Phys. Rev. A 81, 063411 (2010).

64. Fan, H. et al. Atom based RF electric field sensing. J. Phys. B 48, 202001 (2015).

65. Boulmer, J, Camus, P, Gagne, J. M. \& Pillet, P. Laser-microwave ionisation mass spectroscopy of ion Rydberg states: $\mathrm{Ba}^{+}$spectrum. J. Phys. B 20, L143 (1987).

66. Jones, R. R. \& Gallagher, T. F. Observation of $\mathrm{Ba}^{+} n \mathrm{p}_{1 / 2}$ and ngj Rydberg series. J. Opt. Soc. Am. B 6, 1467-1472 (1989). 
67. Lange, V., Khan, M. A., Eichmann, U. \& Sandner, W. Rydberg states of the strontium ion. Z. Phys. D 18, 319-324 (1991).

68. Li, W. \& Lesanovsky, I. Entangling quantum gate in trapped ions via Rydberg blockade. Appl. Phys. B 114, 37-44 (2014).

69. Li, W., Glaetzle, A. W., Nath, R. \& Lesanovsky, I. Parallel execution of quantum gates in a long linear ion chain via Rydberg mode shaping. Phys. Rev. A 87, 052304 (2013).

70. Nath, R et al. Hexagonal plaquette spin-spin interactions and quantum magnetism in a two-dimensional ion crystal. New J. Phys. 17, 065018 (2015).

71. Li, W. \& Lesanovsky, I. Electronically Excited Cold Ion Crystals. Phys. Rev. Lett. 108, 023003 (2012).

72. Feldker, T. et al. Mode shaping in mixed ion crystals of ${ }^{\circ} \mathrm{Ca}^{2+}$ and ${ }^{4} \mathrm{Ca}^{+}$. Appl. Phys. B 114, 11-16 (2014).

73. Feldker, T. et al. Rydberg Excitation of a Single Trapped Ion. Phys. Rev. Lett. 115, 173001 (2015).

74. Bachor, P., Feldker, T., Walz, J. \& Schmidt-Kaler, F. Addressing single trapped ions for Rydberg quantum logic. J. Phys. B 49, 154004 (2016).

75. Feldker, T. Rydberg Excitation of Trapped Ions PhD thesis (Johannes Gutenberg-Universität Mainz, 2017).

76. Schmidt-Kaler, F et al. Rydberg excitation of trapped cold ions: a detailed case study. New J. Phys. 13, 075014 (2011).

77. Aymar, M., Greene, C. H. \& Luc-Koenig, E. Multichannel Rydberg spectroscopy of complex atoms. Rev. Mod. Phys. 68, 10151123 (1996).

78. Šibalić, N., Pritchard, J., Adams, C. \& Weatherill, K. ARC: An open-source library for calculating properties of alkali Rydberg atoms. Comput. Phys. Commun. 220, 319 -331 (2017).

79. Gallagher, T. F. \& Cooke, W. E. Interactions of Blackbody Radiation with Atoms. Phys. Rev. Lett. 42, 835-839 (1979).

8o. Saffman, M., Walker, T. G. \& Mølmer, K. Quantum information with Rydberg atoms. Rev. Mod. Phys. 82, 2313-2363 (2010). 
81. Jaksch, D. et al. Fast Quantum Gates for Neutral Atoms. Phys. Rev. Lett. 85, 2208-2211 (10 2000).

82. Biémont, E. et al. Lifetimes of metastable states in Sr II. Eur. Phys. J. D 11, 355-365 (2000).

83. Safronova, U. I. All-order perturbation calculation of energies, hyperfine constants, multipole polarizabilities, and blackbody radiation shift in ${ }^{87} \mathrm{Sr}^{+}$. Phys. Rev. A 82, 022504 (2010).

84. Letchumanan, V., Wilson, M. A., Gill, P. \& Sinclair, A. G. Lifetime measurement of the metastable $4 \mathrm{~d}^{2} \mathrm{D}_{5 / 2}$ state in ${ }^{88} \mathrm{Sr}^{+}$ using a single trapped ion. Phys. Rev. A 72, 012509 (2005).

85. Pinnington, E. H., Berends, R. W. \& Lumsden, M. Studies of laser-induced fluorescence in fast beams of $\mathrm{Sr}^{+}$and $\mathrm{Ba}^{+}$ions. J. Phys. B 28, 2095 (1995).

86. Zhang, $\mathrm{H}$. et al. Iterative precision measurement of branching ratios applied to $5 \mathrm{P}$ states in ${ }^{88} \mathrm{Sr}^{+}$. New J. Phys. 18, 123021 (2016).

87. Roos, C. Controlling the quantum state of trapped ions $\mathrm{PhD}$ thesis (Universität Innsbruck, 2000).

88. Pokorny, F. Experimental setup for trapping strontium Rydberg ions Master's thesis (Universität Innsbruck, 2014).

89. Guggemos, M. Precision spectroscopy with trapped ${ }^{\circ} \mathrm{Ca}^{+}$and ${ }^{27} \mathrm{Al}^{+}$ions $\mathrm{PhD}$ thesis (Universität Innsbruck, 2017).

90. Chwalla, M. Precision spectroscopy with ${ }^{40} \mathrm{Ca}^{+}$ions in a Paul trap PhD thesis (Universität Innsbruck, 2009).

91. Michaelson, H. B. The work function of the elements and its periodicity. J. Appl. Phys. 48, 4729-4733 (1977).

92. Niedermayr, M. Cryogenic surface ion traps PhD thesis (Universität Innsbruck, 2015).

93. Brownnutt, M. et al. Controlled photoionization loading of ${ }^{88} \mathrm{Sr}^{+}$for precision ion-trap experiments. Appl. Phys. B 87, 411-415 (2007). 
94. Berkeland, D. J., Miller, J. D., Bergquist, J. C., Itano, W. M. \& Wineland, D. J. Minimization of ion micromotion in a Paul trap. J. Appl. Phys. 83, 5025-5033 (1998).

95. Keller, J., Partner, H. L., Burgermeister, T. \& Mehlstäubler, T. E. Precise determination of micromotion for trapped-ion optical clocks. J. Appl. Phys. 118, 104501 (2015).

96. Brandl, M. F. et al. Cryogenic setup for trapped ion quantum computing. Rev. Sci. Instrum. 87, 113103 (2016).

97. Mehta, K. K. et al. Integrated optical addressing of an ion qubit. Nat. Nanotechnol. 11, 1066 (2016).

98. Manovitz, T. et al. Fast Dynamical Decoupling of the MølmerSørensen Entangling Gate. Phys. Rev. Lett. 119, 220505 (2017).

99. Barwood, G. P. et al. Agreement between two ${ }^{88} \mathrm{Sr}^{+}$optical clocks to 4 parts in 10 ${ }^{17}$. Phys. Rev. A 89, 050501 (2014).

10o. Dubé, P., Bernard, J. E. \& Gertsvolf, M. Absolute frequency measurement of the ${ }^{88} \mathrm{Sr}^{+}$clock transition using a GPS link to the SI second. Metrologia 54, 290 (2017).

101. Kolachevsky, N., Alnis, J., Bergeson, S. D. \& Hänsch, T. W. Compact solid-state laser source for $1 \mathrm{~S}-2 \mathrm{~S}$ spectroscopy in atomic hydrogen. Phys. Rev. A 73, 021801 (2006).

102. Maier, C. Laser system for the Rydberg excitation of Strontium ions Master's thesis (Universität Innsbruck, 2013).

103. Wilson, A. C. et al. A 750-mW, continuous-wave, solid-state laser source at $313 \mathrm{~nm}$ for cooling and manipulating trapped ${ }^{9} \mathrm{Be}^{+}$ions. Appl. Phys. B 105, 741-748 (2011).

104. Lo, H.-Y. et al. All-solid-state continuous-wave laser systems for ionization, cooling and quantum state manipulation of beryllium ions. Appl. Phys. B 114, 17-25 (2014).

105. Donley, E. A., Heavner, T. P., Levi, F., Tataw, M. O. \& Jefferts, S. R. Double-pass acousto-optic modulator system. Rev. Sci. Instrum. 76, 063112 (2005).

106. Colombe, Y., Slichter, D. H., Wilson, A. C., Leibfried, D. \& Wineland, D. J. Single-mode optical fiber for high-power, low-loss UV transmission. Opt. Express 22, 19783 (2014). 
107. Haag, J. Glasfasern als Wellenleiter für ultraviolettes Licht Master's thesis (Universität Innsbruck, 2015).

108. Drever, R. W. P. et al. Laser phase and frequency stabilization using an optical resonator. Appl. Phys. B 31, 97-105 (1983).

109. Black, E. D. An introduction to Pound-Drever-Hall laser frequency stabilization. Am. J. Phys. 69, 79-87 (2001).

110. Kumph, M. 2D Arrays of Ion Traps for Large Scale Integration of Quantum Information Processors PhD thesis (Universität Innsbruck, 2015).

111. Brandl, M. Towards Cryogenic Scalable Quantum Computing with Trapped Ions PhD thesis (Universität Innsbruck, 2017).

112. Ludlow, A. D. et al. Compact, thermal-noise-limited optical cavity for diode laser stabilization at $1 \times 10^{-15}$. Opt. Lett. 32, 641643 (2007).

113. Kress, F. Frequenzstabilisierung eines $674 \mathrm{~nm}$ Diodenlasers zur Detektion der Rydberganregung von Strontiumionen Master's thesis (Universität Innsbruck, 2015).

114. Benhelm, J. Precision Spectroscopy and Quantum Information Processing with Trapped Calcium Ions PhD thesis (Universität Innsbruck, 2008).

115. Kirchmair, G. Quantum non-demolition measurements and quantum simulation PhD thesis (Universität Innsbruck, 2010).

116. Pham, P. T. T. A general-purpose pulse sequencer for quantum computing Master's thesis (Massachusetts Institute of Technology, 2005).

117. Schindler, P. Frequency synthesis and pulse shaping for quantum information processing with trapped ions Master's thesis (Universität Innsbruck, 2008).

118. Schreck, F. Control System http : / / www . strontiumbec . com/. Accessed: 20/11/2017. (2015).

119. Audi, G., Bersillon, O., Blachot, J. \& Wapstra, A. The Nubase evaluation of nuclear and decay properties. Nucl. Phys. A 729, 3 -128 (2003). 
120. Leibrandt, D. R. et al. Laser ablation loading of a surfaceelectrode ion trap. Phys. Rev. A 76, 055403 (2007).

121. Łabaziewicz, J. High Fidelity Quantum Gates with Ions in Cryogenic Microfabricated Ion Traps PhD thesis (Massachusetts Institute of Technology, 2008).

122. Ofek, N. et al. Extending the lifetime of a quantum bit with error correction in superconducting circuits. Nature 536, 441 (2016).

123. Saffman, M. \& Walker, T. G. Analysis of a quantum logic device based on dipole-dipole interactions of optically trapped Rydberg atoms. Phys. Rev. A 72, 022347 (2005).

124. Potvliege, R. M. \& Adams, C. S. Photo-ionization in far-offresonance optical lattices. New J. Phys. 8, 163 (2006).

125. Johnson, T. A. et al. Rabi Oscillations between Ground and Rydberg States with Dipole-Dipole Atomic Interactions. Phys. Rev. Lett. 100, 113003 (2008).

126. Beterov, I. I., Tretyakov, D. B., Ryabtsev, I. I., Ekers, A. \& Bezuglov, N. N. Ionization of sodium and rubidium $n S, n P$, and $n \mathrm{D}$ Rydberg atoms by blackbody radiation. Phys. Rev. A 75, 052720 (2007).

127. James, D. Quantum dynamics of cold trapped ions with application to quantum computation. Appl. Phys. B 66, 181-19o (1998).

128. Home, J. P., Hanneke, D, Jost, J. D., Leibfried, D \& Wineland, D. J. Normal modes of trapped ions in the presence of anharmonic trap potentials. New J. Phys. 13, 073026 (2011).

129. Nägerl, H. C., Leibfried, D., Schmidt-Kaler, F., Eschner, J. \& Blatt, R. Coherent excitation of normal modes in a string of $\mathrm{Ca}^{+}$ions. Opt. Express 3, 89-96 (1998).

130. Higgins, G. et al. Single Strontium Rydberg Ion Confined in a Paul Trap. Phys. Rev. X 7, 021038 (2017).

131. Itano, W. M. External-field shifts of the ${ }^{199} \mathrm{Hg}^{+}$optical frequency standard. J. Res. Nat. Inst. Stand. Technol. 105 (2000). 
132. Shaniv, R., Akerman, N. \& Ozeri, R. Atomic Quadrupole Moment Measurement Using Dynamic Decoupling. Phys. Rev. Lett. 116, 140801 (2016).

133. Jiang, D., Arora, B. \& Safronova, M. S. Electric quadrupole moments of metastable states of $\mathrm{Ca}^{+}, \mathrm{Sr}^{+}$, and $\mathrm{Ba}^{+}$. Phys. Rev. A 78, 022514 (2008).

134. Friedrich, H. Theoretical Atomic Physics (Springer International Publishing, 2017).

135. Bain, A. D. \& Dumont, R. S. Introduction to Floquet theory: The calculation of spinning sideband intensities in magic-angle spinning NMR. Concepts Magn. Reson. 13, 159-170 (2001).

136. Fleischhauer, M., Imamoglu, A. \& Marangos, J. P. Electromagnetically induced transparency: Optics in coherent media. Rev. Mod. Phys. 77, 633-673 (2005).

137. Higgins, G., Pokorny, F., Zhang, C., Bodart, Q. \& Hennrich, M. Coherent Control of a Single Trapped Rydberg Ion. Phys. Rev. Lett. 119, 220501 (2017).

138. Sillanpää, M. A. et al. Autler-Townes Effect in a Superconducting Three-Level System. Phys. Rev. Lett. 103, 193601 (2009).

139. Foot, C. Atomic Physics (Oxford University Press, 2005).

140. Johansson, J., Nation, P. \& Nori, F. QuTiP 2: A Python framework for the dynamics of open quantum systems. Comput. Phys. Commun. 184, $1234-1240$ (2013).

141. Vitanov, N. V., Rangelov, A. A., Shore, B. W. \& Bergmann, K. Stimulated Raman adiabatic passage in physics, chemistry, and beyond. Rev. Mod. Phys. 89, 015006 (2017).

142. Bergmann, K., Vitanov, N. V. \& Shore, B. W. Perspective: Stimulated Raman adiabatic passage: The status after 25 years. $J$. Chem. Phys. 142, 170901 (2015).

143. Cubel, T. et al. Coherent population transfer of ground-state atoms into Rydberg states. Phys. Rev. A 72, 023405 (2005).

144. Deiglmayr, J. et al. Coherent excitation of Rydberg atoms in an ultracold gas. Opt. Commun. 264, 293-298 (2006). 
145. Sparkes, B. M. et al. Stimulated Raman adiabatic passage for improved performance of a cold-atom electron and ion source. Phys. Rev. A 94, 023404 (2016).

146. Berry, M. V. Quantal phase factors accompanying adiabatic changes. Proc. Royal Soc. A 392, 45-57 (1984).

147. Yale, C. G. et al. Optical manipulation of the Berry phase in a solid-state spin qubit. Nat. Photonics 10, 184-189 (2016).

148. Zanardi, P. \& Rasetti, M. Holonomic quantum computation. Phys. Lett. A 264, 94 -99 (1999).

149. Duan, L.-M., Cirac, J. I. \& Zoller, P. Geometric Manipulation of Trapped Ions for Quantum Computation. Science 292, 16951697 (2001).

150. Chuang, I. L. \& Nielsen, M. A. Prescription for experimental determination of the dynamics of a quantum black box. J. Mod. Opt. 44, 2455-2467 (1997).

151. James, D. F. V., Kwiat, P. G., Munro, W. J. \& White, A. G. Measurement of qubits. Phys. Rev. A 64, 052312 (2001).

152. Møller, D., Madsen, L. B. \& Mølmer, K. Geometric phase gates based on stimulated Raman adiabatic passage in tripod systems. Phys. Rev. A 75, 062302 (2007).

153. Rao, D. D. B. \& Mølmer, K. Robust Rydberg-interaction gates with adiabatic passage. Phys. Rev. A 89, 030301 (2014). 
